\title{
Three Essays on the Study of Persistence via Impulse-Response Confidence Bands with Application to Autoregressive Moving Average (ARMA) Processes
}

by

\author{
Beatriz Peraza López
}

A thesis submitted to the Faculty of Graduate and Postdoctoral Affairs

in partial fulfillment of the requirements for the degree of

\section{Doctor of Philosophy}

in

Economics

Department of Economics

Carleton University

Ottawa, Ontario, Canada

(C)2016, Beatriz Peraza 


\section{Abstract}

The two-step simulation-based method introduced in this thesis combines Indirect Inference jointly with Monte Carlo (MC) test methods to deliver identification robust confidence sets for finite samples. In the first chapter, a general framework is introduced, which considers three auxiliary estimating functions: two-sided forward and backward looking regressions with leads and lags, long- autoregressions and empirical autocorrelations. The first simulation stage is incorporated to calibrate parameter estimates. In the second simulation stage, the resulting objective functions are inverted applying the MC test to obtain joint confidence sets. The empirical focus is on Autoregressive Moving Average (ARMA) processes, since identification and boundary issues raise enduring complications for estimation and inference. In particular, simultaneous impulse-responses confidence bands are derived for ARMA processes via confidence set projections. Supporting simulation studies illustrate the accurate size and good power of our method.

The following chapters introduce further refinements to our framework with empirical applications. The second chapter investigates the persistence of oil shocks via impulse-response confidence bands for two crude oil benchmarks priced in U.S. dollars per barrel: the West Texas Intermediate (WTI) for high quality light sweet crude oil delivered at Cushing, Oklahoma, and the Western Canadian Select (WCS) for heavy blended high-TAN (acidic) crude, quoted at the Husky terminal in Hardisty, Alberta. Stable distributions are incorporated to be able to capture the heavy tails and asym- 
metry encountered. In addition, our framework remains agnostic with respect to the process followed by oil returns by allowing for processes with no persistence, spurious white noise related to root cancelation, heavy persistence, as well as almost unit root. For the particular case of non-Gaussian Stable distribution, the "Local" Monte Carlo (LMC) test method is applied in the second stage, which is based on calibrations of the nuisance parameters of skewness and kurtosis. By studying long and short weekly samples as well as monthly samples, we find that shocks to oil returns are not permanent, since they are able to dissipate in short to middle time frames of approximately twenty periods. We conclude that allowing for asymmetry helps disentangle the persistence of oil shocks. Our findings support the usefulness of impulse-responses obtained from asymmetric and heavy tailed distributions as a tool to understand the uncertainty surrounding commonly applied forecasting techniques.

Lastly, the third chapter assesses the persistence of shocks on a measure of idiosyncratic risk (MIR) computed for a non-diversifiable portfolio containing common stocks of the three major Telecom carriers in Canada. Our methodology offers the advantage of isolating the idiosyncratic risk to be able to understand the persistence of shocks and spillovers at sector-specific levels with a univariate approach. The twostage simulation based method is applied with a particular refinement; the average of the statistics of the three estimating functions is computed as a forth resulting test statistic to harvest information from the other three. We conclude that a shock to the MIR for the Canadian Telecom portfolio can be described as having a shortlived large impact and relatively small oscillatory effects, which remain close to zero approximately after eight months. 
To my mother, Irma López Saura,

for her love, inspiration and support. 


\section{Acknowledgements}

I would like to express my sincere gratitude to my thesis supervisor and mentor, Professor Lynda Khalaf, for her invaluable guidance and encouragement throughout this learning process. Her incessant enthusiasm and passion for econometrics provided great inspiration to my research. Moreover, her personal kindness and generosity made me look forward to every meeting.

I would like to thank my committee members, Professors Marcel Voia from Carleton University and Maral Kichian from University of Ottawa for their helpful comments and support along the way. I greatly benefited from the econometric courses thought by Marcel Voia during my master and doctoral training. I am thankful to Maral Kichian for our conversations on econometrics and her generous advices on career paths.

I would like to express my appreciation to the Department of Economics and the Faculty of Graduate and Postdoctoral Affairs at Carleton University for providing financial support during my studies. I am thankful to many faculty members and administrative staff for their encouragement and support.

I would also like to thank Professor Gennady Shaikhet from the Schools of Mathematics and Statistics at Carleton University. His probability, finance and simulation courses were a source of inspiration to my research.

Many thanks to Larry Shute and Pierre Therrien at Industry Canada for the opportunity of developing a research paper, which became the last chapter of this 
thesis.

I am grateful to my fellow classmates and colleagues, especially Anand Acharya, Abber Reza and Zhenjiang Lin for their support and generous advice.

Finally, my deepest gratitude goes to my mother, Irma López Saura and my husband, Julian R. Solano Acosta for their love, patience, support and encouragement along the way. 


\section{Table of Contents}

$\begin{array}{ll}\text { Abstract } & \text { ii }\end{array}$

Acknowledgements $\quad$ v

1 Simultaneous Indirect Inference, Impulse Responses, and ARMA models 1

1.1 Introduction . . . . . . . . . . . . . . . . . . . . 1

1.2 General Framework . . . . . . . . . . . . . . . . . . 7

1.2.1 Nuisance parameters . . . . . . . . . . . . . . . . . 8

1.3 Inference via test inversion . . . . . . . . . . . . . . . . . . 11

1.3.1 Exact $p$-values . . . . . . . . . . . . . . . . 13

1.4 ARMA special case . . . . . . . . . . . . . . . . . . . 14

1.4.1 Impulse-response confidence bands . . . . . . . . . . . . . 16

1.5 Simulation Study . . . . . . . . . . . . . . . . . . . . 17

1.5.1 Robustness checks . . . . . . . . . . . . . . 21

1.5.2 Impulse-response confidence bands . . . . . . . . . . . . . . . 22

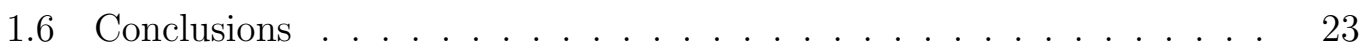

$\begin{array}{ll}\text { References } & 24\end{array}$

2 Projection- Based Impulse-Response Confidence Bands for Noth American Crude Oil Benchmarks 45

2.1 Introduction . . . . . . . . . . . . . . . . . . 45

2.2 Framework . . . . . . . . . . . . . . . . . . 51

2.2.1 Assumptions . . . . . . . . . . . . . . . . 51

2.2.2 Two-Stage Simulation-Based Method . . . . . . . . . . 54

2.3 Data . . . . . . . . . . . . . . . . . . . . 58

2.4 Results . . . . . . . . . . . . . . . . . 60

2.4.1 West Texas Intermediate (WTI) . . . . . . . . . . . . . . 62

2.4.2 Western Canadian Select (WCS) . . . . . . . . . . 64 
2.5 Conclusion . . . . . . . . . . . . . . . . . 66

$\begin{array}{ll}\text { References } & 68\end{array}$

3 The Persistence of Idiosyncratic Shocks in Non-Diversifiable Portfolios $\quad 93$

3.1 Introduction . . . . . . . . . . . . . . . . . . . . . . . 93

3.2 Framework . . . . . . . . . . . . . . . . . . . . 100

3.2.1 Idiosyncratic Risk . . . . . . . . . . . . . . . . . . . 100

3.2 .2 Assumptions . . . . . . . . . . . . . . . . . . . . . . 102

3.2.3 Two-Stage Simulation-Based Method . . . . . . . . . . . . . . 102

3.3 Data . . . . . . . . . . . . . . . . . . . 106

3.4 Results . . . . . . . . . . . . . . . . . . . . . . . . 110

3.5 Conclusion . . . . . . . . . . . . . . . . . . . . . 113

$\begin{array}{ll}\text { References } & 115\end{array}$ 


\section{List of Tables}

1.1 Empirical Rejections: True value $\theta_{0}=0.6 \ldots \ldots$. . . . . . . . . 30

1.2 Empirical Rejections: True value $\theta_{0}=0.99 \ldots \ldots 31$

1.3 Empirical Rejections: True pair $\left\{\theta_{0}, \psi_{0}\right\}=\{0,0.6\} \ldots . \ldots . \ldots 32$

1.4 Empirical Rejections: True pair $\left\{\theta_{0}, \psi_{0}\right\}=\{0.5,0.96\} \ldots 33$

1.5 Empirical Rejections: True pair $\left\{\theta_{0}, \psi_{0}\right\}=\{0.96,0.5\} \ldots \ldots 34$

1.6 Empirical Rejections: True pair $\left\{\theta_{0}, \psi_{0}\right\}=\{0.65,-0.65\} \ldots 35$

1.7 Empirical Rejections: True pair $\left\{\theta_{0}, \psi_{0}\right\}=\{0.99,0.99\} \ldots 37$

1.8 Empirical Rejections: True values $\left\{\theta_{1,0}, \theta_{2,0}, \psi_{0}\right\}=\{0.3,0.85,0.60\} \quad .38$

1.9 Empirical Rejections: True values $\left\{\theta_{0}, \psi_{1,0}, \psi_{2,0}\right\}=\{0.3,0.6,0.85\} \quad$. 38

2.1 Returns, Summary of Statistics _. . . . . . . . . . . . . . . 60

3.1 Excess Returns: Summary of Statistics . . . . . . . . . . . . . . . 107

3.2 Measure of Idiosyncratic Risk: Summary of Statistics . . . . . . . . . 109 


\section{List of Figures}

1.1 ARMA (1,1) Simulated Path $\left\{\theta_{0}, \psi_{0}\right\}=\{0.99,0.99\} \quad \ldots \ldots 39$

1.2 ARMA (1,1) Simulated Path $\left\{\theta_{0}, \psi_{0}\right\}=\{0.96,0.5\} \ldots \ldots . \ldots 41$

1.3 ARMA (1,1) Simulated Path $\left\{\theta_{0}, \psi_{0}\right\}=\{0.65,-0.65\} \ldots \ldots 43$

2.1 West Texas Intermediate . . . . . . . . . . . . . . . . . . . . 74

2.2 Western Canadian Select . . . . . . . . . . . . . . . . . . 74

2.3 West Texas Intermediate . . . . . . . . . . . . . . . . . . 75

2.4 Western Canadian Select . . . . . . . . . . . . . . . . . . 75

2.5 West Texas Intermediate: Kernel Density Estimate . . . . . . . . . . 76

2.6 Western Canadian Select: Kernel Density Estimate . . . . . . . . . 76

2.7 West Texas Intermediate (WTI) Jan. 1986- Dec. 2014 (Weekly). Gaussian Distribution . . . . . . . . . . . . . . 77

2.8 West Texas Intermediate (WTI) Jan. 1986- Dec. 2014 (Weekly). Stable Non-Gaussian distribution . . . . . . . . . . . . . . . 79

2.9 West Texas Intermediate (WTI) Jan. 1986- Dec. 2014 (Monthly). Student-t Distribution with 8 Degrees of Freedom . . . . . . . . . . 81

2.10 West Texas Intermediate (WTI) Jan. 2010- Dec. 2014 (Weekly). Student-t Distribution with 8 Degrees of Freedom . . . . . . . . . 83

2.11 Western Canadian Select (WCS) May 2008 Dec. 2014 (Weekly). Gaussian Distribution . . . . . . . . . . . . . . . . . 85

2.12 Western Canadian Select (WCS) May 2008 Dec. 2014 (Weekly). Stable Non-Gaussian Distribution _ . . . . . . . . . . . . . 87

2.13 Western Canadian Select (WCS) May 2008 Dec. 2014 (Monthly). Gaussian Distribution . . . . . . . . . . . . . . . 89

2.14 Western Canadian Select (WCS) Jan. 2010- Dec. 2014 (Weekly). Gaussian Distribution . . . . . . . . . . . . . . . . . . . . . 91

3.1 S\&P TSX: Histogram and Kernel Density Estimate . . . . . . . . . . 118

3.2 BCE (BCE): Histogram and Kernel Density Estimate . . . . . . . . . 118

3.3 Rogers (RCI.B): Histogram and Kernel Density Estimate . . . . . . . 119 
3.4 Telus (T): Histogram and Kernel Density Estimate . . . . . . . . . . 119

3.5 Measure of Idiosyncratic Risk . . . . . . . . . . . . . . . . . . . 120

3.6 Detrended MIR Jan. 2005 -Dec. 2014.

Estimating function: OLS-long-AR . . . . . . . . . . . . . . 121

3.7 Detrended MIR Jan. 2005 -Dec. 2014.

Estimating function: Autocorrelations . . . . . . . . . . . . . . . 123

3.8 Detrended MIR Jan. 2005 -Dec. 2014. Averaging Method . . . . . . 125 


\section{Chapter 1}

\section{Simultaneous Indirect Inference,}

\section{Impulse Responses, and ARMA}

\section{models}

\subsection{Introduction}

Indirect inference refers to resampling-based statistical methods that use simulation to calculate estimating functions including likelihoods, scores or moments. The idea of replacing complicated or intractable statistical functions by computer simulations motivated initial applications and underlying theory; see Smith (1993), Gouriéroux, Monfort and Renault (1993), Gouriéroux and Monfort (1996) and Gallant and Tauchen (1996). Since then, available evidence suggests that indirect inference often works better than traditional methods in finite samples for bias or misspecification corrections under various situations. ${ }^{1}$ This chapter considers a two-stage framework that can yield identification robust confidence sets via Indirect Inference.

\footnotetext{
${ }^{1}$ See, for example, Robins, Van der Vaart and Ventura (2000), Ronchetti and Trojani (2001), Calzolari, Fiorentini and Sentana (2004) ,Dridi, Renault and Guay (2007), Gouriéroux, Phillips and Yu (2010), Li (2010), Dominicy and Veredas (2013), Fuleky and Zivot (2014), Calvet and Czellar (2015), and the working papers by Chaudhuri et al. (2015) and Forneron and Ng (2015).
} 
The primary focus concerns inference in Autoregressive Moving Average (ARMA) models including impulse responses, in which case we derive simultaneous confidence bands, as in Jordá (2009); see also Jordá and Marcellino (2010) and Jordá, Knuppel and Marcellino (2013) on simultaneous path forecasts.

Despite the simplicity of ARMA models in time series, identification and boundary issues raise enduring complications for estimation and inference. Autoregression (AR) unit roots cause the so-called non-uniform convergence problem: methods that require stationarity break down at or close to the unit boundary, whereas local-tounity motivated methods perform poorly away from unity; see Hansen (1999), Mikusheva (2007), Andrews and Guggenberger (2010), and more recently, Gorodnichenko, Mikusheva and Ng (2012), Mikusheva (2012, 2014) and Phillips (2014). Invertibility of Moving Average (MA) components causes the so-called pile up effect: because of identification failure, estimating functions including likelihoods can have a local maximum at the invertibility boundary even when the true process is invertible; see Sargan and Bhargava (1983), Davis and Dunsmuir (1996), Gospodinov (2002), Genton and Ronchetti (2003), Billio and Monfort (2003) and more recently, Davis and Song (2011), and Gospodinov and Ng (2015). Root cancelation, also known as common factors, or parameter redundancy, described in Harvey (1981), occurs when the AR polynomial and the MA polynomial have one or more roots in common. Root cancelation and near-root cancelation produce spurious inference related to lack of identification or weak-identification of the parameters in question. Inference concerns in the presence of root cancelation have been extensively described and addressed in the literature. For instance, Ansley and Newbold (1980) use simulations to analyze the properties of MLE, exact and conditional least squares considering stationary ARMA models with sample sizes of 50 and 100 observations. They document the severe bias suffered by MLE estimates in the presence of root cancelation, since confidence intervals are too narrow. Galbraith and Zinde-Walsh (1997) and references therein 
focus on providing identification devices for ARMA models. Their proposed test for common factors involves computing a Wald statistic to test whether the determinant of a partitioned matrix containing approximate autoregressive coefficients is zero. Nelson and Startz (2007) introduce the zero-information-limit-condition (ZILC) as a tool to recognize models with weak- identification, which affects standard inference statistics such as asymptotic Student-t tests. Among other examples, they discuss the weak-identification case for ARMA $(1,1)$ in a simulation with a sample size of 1000 observations. The authors recommend practitioners to be cautious, since near-root cancelations lead to spurious inference regardless of large sample sizes, even when the asymptotic theory is expected to hold. Andrews and Cheng (2012) introduce an asymptotic framework with a drifting parameter approach to obtain identificationrobust estimates, tests and confidence sets, which applies to a class of extremum estimators satisfying a set of criterion function (stochastic) quadratic approximation conditions. The group of estimators considered includes MLE, least squares, generalized method of moments (GMM), generalized empirical likelihood, minimum distance and semi-parametric estimators. Their methodology tackles the weak -identification region as well as the semi-strong identification region, a middle point between weak and strong identification. It requires the computation of quadratic expansions under certain conditions to address the problems faced by standard asymptotic approximations in flat segments of the functions in question, which produce a non-invertible second derivative matrix. Their methodology is applied to $\operatorname{ARMA}(1,1)$ with common roots estimated by (quasi) MLE and they examine Student-t and quasi likelihood ratio (QLR) tests. Their robust confidence intervals have asymptotic and finite sample sizes $^{2}$ that are equal and close to 0.95 , respectively, and the best power is the one based on the QLR test statistic. Recently Andrews and Mikusheva (2014) introduce a measure to capture the disparity between the normalized quadratic variation of the

\footnotetext{
${ }^{2}$ Throughout this document, size and coverage are used interchangeable; see Andrews and Cheng (2012).
} 
score, which converges to the normalized Fisher information matrix, and the normalized negative Hessian of the log-likelihood, which remains volatile for large samples. Using simulations with a stylized Dynamic Stochastic General Equilibrium (DSGE) model, they show that a large measure indicates distortions to the distribution of the MLE due to weak-identification problems. In a follow up research with focus on identification-robust Lagrange multiplier (score), Andrews and Mikusheva (2015) refer to ARMA processes with near-root cancelation as part of the DESG context and prove that weak-identification robust score test statistics converge to chi-squared distribution. Our approach complements the asymptotic frameworks available in the literature in the sense that it presents a finite sample version. To the best of our knowledge our research introduces a finite sample and impulse responses perspective, which are not pursued in the aforementioned papers. We propose confidence sets robust to these problems via Indirect Inference and the following auxiliary estimates: (i) two-sided forward and backward looking regressions with leads and lags, (ii) long-autoregressions (AR), and (iii) empirical autocorrelations.

Methods with leads and lags generally aim to deal with feedback effects; see Stock and Watson (1993), Dufour and Torrès (2000). AR-based approximations have long been popular for ARMA models; see Hannan and Rissanen (1982), Galbraith and Zinde-Walsh (1994, 1997), Ghysels, Khalaf and Vodounou (2002), Guay and Scaillet (2003), Gospodinov and Ng (2015), Dufour and Pelletier (2014). Empirical autocorrelations and related measures including cumulants and local projections have recently been re-introduced from a minimum distance perspective; see e.g. Jordá and Kozicki (2011), Gorodnichenko, Mikusheva and Ng (2012), Gospodinov and Ng (2015) and the references therein. The indirect inference framework we propose unifies all the above for confidence interval purposes.

While the ARMA case concretizes concepts, our method applies more generally, as follows. Indirect inference relies on a binding function, denoted thereafter $\lambda=\mathcal{L}(\beta)$, 
that links a parameter of interest $\beta$ to an auxiliary parameter $\lambda$ for which a simple estimator is available. Denote the latter $\hat{\lambda}=\hat{\Lambda}(X)$ where $X$ refers to available data and $\hat{\Lambda}($.$) is the estimating function. \mathcal{L}(\beta)$ need not be known but admits a simulationbased approximate. Denote the latter $\Lambda(\beta)$. Conformably, a minimum-distance estimator is derived that matches $\hat{\lambda}$ to $\Lambda(\beta)$. To set focus, consider a Wald-type distance metric of the form $S(X, \beta)=(\hat{\lambda}-\Lambda(\beta))^{\prime}(\hat{\lambda}-\Lambda(\beta))$. The standard Indirect Inference estimator corresponds to $\hat{\beta}=A R G M I N_{\beta}(S(X, \beta))$. Associated confidence sets and hypotheses tests are typically justified using standard asymptotic arguments. For example, regularity conditions are derived to ensure consistency and e.g. asymptotic normality of $\hat{\beta}$, leading to Wald-type confidence intervals. Such intervals usually require identifying $\beta$, which may be an issue in many models that necessitate Indirect Inference. We revisit Indirect Inference from an alternative outlook, to demonstrate its usefulness when $\beta$ is hard to identify and when further parameters of interest are $m$ non-standard transformations of $\beta$, as with impulse responses, which we denote $g_{i}(\beta), i=1, \ldots, m$.

In contrast to the above described extremum estimation strategy, we treat $S(X, \beta)$ as a test statistic which we invert rather than minimize. Inverting a test involves collecting the parameter values that are not rejected via this test at a given level of significance $\alpha$ to construct a confidence set. We propose to automate the latter into Indirect Inference, along these lines. Assume that $\Lambda(\beta)$ can be derived by simulating the true process once $\beta$ is set to a given value, say $\beta_{0}$. Then a simulation-based $p$-value can be derived for $S\left(X, \beta_{0}\right)=\left(\hat{\lambda}-\Lambda\left(\beta_{0}\right)\right)^{\prime}\left(\hat{\lambda}-\Lambda\left(\beta_{0}\right)\right)$ using this same $\beta_{0}$ and a supplementary simulation round conditional on $\Lambda\left(\beta_{0}\right)$. Denote the $p$-value thus derived as $\hat{p}\left(\beta_{0}\right)$. Simulations are thus incorporated in two stages, first to calibrate $\Lambda\left(\beta_{0}\right)$ in $S\left(X, \beta_{0}\right)$ and next to compute its $p$-value. An $\alpha$-level joint confidence set can thus be assembled by numerically collecting the $\beta_{0}$ values for which $\hat{p}\left(\beta_{0}\right)>\alpha$.

Exactness and identification robustness are achieved by computing $\hat{p}\left(\beta_{0}\right)$ via the 
MC test method of Dufour (2006). This method achieves size control when the null distribution of the statistic, even if not computable analytically, does not depend on unknown or nuisance parameters. In this regard, our work relates to Dufour and Valery $(2006,2009)$ in stochastic volatility models, and Dufour and Kurz-Kim (2010) and Beaulieu, Dufour and Khalaf (2007, 2013, 2014) in models with fat-tailed fundamentals. Many applications of Indirect Inference satisfy this requirement nearly by definition, as $\Lambda(\beta)$ should be computable once $\beta$ is set. In our ARMA application, a nuisance-parameter-free distribution results from scale-invariance of OLS in AR settings, and from the standardization inherent to empirical autocorrelations. ${ }^{3}$ For inference on $g_{i}(\beta), i=1, \ldots, m$, the join confidence set is projected, which entails maximizing and minimizing each function over the non-rejected values of $\beta$. The confidence intervals obtained are thus simultaneous by construction and robust to the aforementioned identification problems.

We conduct a simulation study on ARMA and MA processes and compare our method with MLE-based inference. Simple objective functions as in Galbraith and Zinde-Walsh $(1994,1997)$ as well as over-identified criteria are considered, in addition to various lag structures. Results can be summarized as follows. In contrast to MLE, our method eradicates pile up problems even with very small samples, with no more than 50 observations. The significance level is affected neither by the flat segments in the objective function, nor by how close the estimated parameters are to one. MLE is oversized for both individual parameter and joint tests. None of the estimation functions steadily outperforms the rest for all sample sizes and pairs tested. Leads improve power for highly persistent processes. Overidentification yields important power benefits. On balance, autocorrelation-based methods require a larger sample to catch up with AR-based counterparts. Inverting the asymptotic Wald test based

\footnotetext{
${ }^{3}$ Scale invariance is not an unduly restrictive requirement particularly in univariate models. For example, Gouriéroux, Phillips and Yu (2010) consider a unit variance for Indirect Inference on the lag coefficient in dynamic Panels; Beaulieu, Dufour and Khalaf (2014) standardize scale exactly to one for MC tests on tail parameters of Stable distributions.
} 
on MLE estimates is almost infeasible at corner solutions with parameters close to the unit boundary, since in many instances the variance-covariance matrix is not invertible. This result is expected and provides support to our research. Finally, we show that MLE-based impulse-responses can severely underestimate the effect of shocks.

This chapter is organized as follows. In section 1.2, we introduce our framework. In section 3, we discuss inference by test inversion. The ARMA special case is presented in section 4. Simulation results are presented in section 5. We conclude in section 6 .

\subsection{General Framework}

Consider the general model

$$
(\mathcal{X},\{\mathcal{P}(\vartheta): \vartheta \in \Theta\})
$$

where $\mathcal{X}$ is the sample space,

$$
X=\left(X_{1}, \ldots, X_{t}, \ldots, X_{T}\right)^{\prime}
$$

represents a sample of size $T, \mathcal{P}(\vartheta)$ is a probability distribution over $\mathcal{X}$ indexed by

$$
\vartheta=\left(\beta^{\prime}, \sigma^{\prime}\right)^{\prime}, \quad \beta \in \Theta_{\beta}, \quad \sigma \in \Theta_{\sigma}
$$

and $\beta$ is the $q$-dimensional parameter of interest. $\sigma$ is a nuisance parameter one aims to partial out.

Pseudo-data can be simulated from (1.2.1) and an auxiliary parameter denoted $\lambda$, of dimension $p \geq q$, can be defined so that it is linked to $\beta$ via an exact or limiting binding function $\lambda=\mathcal{L}(\vartheta)$. In general, $\mathcal{L}($.$) need not have a known analytical form.$ 
Furthermore, a simple statistic is available to estimate $\lambda$ given $X$.

Denote by $\hat{\lambda}=\hat{\Lambda}(X)$ the considered data-based estimate of $\lambda$ where $\hat{\Lambda}($.$) is the$ estimating function. Its simulation-based counterpart given a specific value of $\vartheta$ can be derived, for example, by: (i) creating $H$ simulated paths

$$
x_{h}(\vartheta)=\left(x_{h, 1}(\vartheta), \ldots, x_{h, t}(\vartheta) \ldots, x_{h, T}(\vartheta)\right)^{\prime}, \quad h=1, \ldots, H
$$

each of size $T$ from (1.2.1) given $\vartheta$; (ii) applying the same estimation method that was used to derive $\hat{\lambda}$ to each simulated path, leading to a series of estimators

$$
\hat{\lambda}_{\boldsymbol{h}}(\vartheta)=\hat{\Lambda}\left(x_{h}(\vartheta)\right), \quad h=1, \ldots, H
$$

and then (iii) computing their average:

$$
\Lambda(\vartheta)=\frac{1}{H} \sum_{h=1}^{H} \hat{\lambda}_{\boldsymbol{h}}(\vartheta)
$$

The distance between $\hat{\lambda}$ and $\Lambda(\vartheta)$ forms the basis of Indirect Inference. A common special case is the Wald-type distance measure $(\hat{\lambda}-\Lambda(\vartheta))^{\prime} \hat{\Omega}(\hat{\lambda}-\Lambda(\vartheta))$ where $\hat{\Omega}$ is a positive definite weighting matrix. Gouriéroux et al. (1993) suggest $\hat{\Omega}=I_{p}$ where $I_{p}$ denotes a $p$-dimensional identity matrix because the loss in efficiency with respect to an optimal estimator can be disregarded. This practice is also suggested, more recently, by Gouriéroux, Phillips and Yu (2010).

\subsubsection{Nuisance parameters}

Focusing on $\beta$ as the parameter of interest requires a strategy to account for $\sigma$. In some settings, $\sigma$ can be easily estimated given $\beta$. Alternatively, an auxiliary estimate or objective function that is exactly invariant to $\sigma$ can be considered. We emphasize the latter case in this study, defined via the following assumptions that characterize 
the model and considered estimating functions.

Assumption 1. In the context of model (1.2.1), an estimating function $\hat{\Lambda}($.$) is$ invariant to $\sigma$, if

$$
\hat{\Lambda}\left(X^{*}\right)=\hat{\Lambda}\left(X^{\dagger}\right)
$$

where $X^{*}$ and $X^{\dagger}$ are samples from (1.2.1) conformable with probability distributions $\mathcal{P}\left(\left(\beta^{\prime}, \sigma_{*}^{\prime}\right)^{\prime}\right)$ and $\mathcal{P}\left(\left(\beta^{\prime}, \sigma_{\dagger}^{\prime}\right)^{\prime}\right)$, respectively, and $\sigma_{*} \neq \sigma_{\dagger}$ are two different values of $\sigma$.

This assumption ensures that

$$
\hat{\Lambda}\left(x_{h}\left(\left(\beta^{\prime}, \sigma_{*}^{\prime}\right)^{\prime}\right)\right)=\hat{\Lambda}\left(x_{h}\left(\left(\beta^{\prime}, \sigma_{\dagger}^{\prime}\right)^{\prime}\right)\right), h=1, \ldots, H
$$

which yields an invariant objective function for the transformation that maps $X^{*}$ into $X^{\dagger}$. Indeed, applying Assumption 1 to the calibration in (1.2.6), we have:

$$
\begin{aligned}
\Lambda\left(\left(\beta^{\prime}, \sigma_{*}^{\prime}\right)^{\prime}\right) & =\frac{1}{H} \sum_{h=1}^{H} \hat{\lambda}_{\boldsymbol{h}}\left(\left(\beta^{\prime}, \sigma_{*}^{\prime}\right)^{\prime}\right) \\
& =\frac{1}{H} \sum_{h=1}^{H} \hat{\lambda}_{\boldsymbol{h}}\left(\left(\beta^{\prime}, \sigma_{\dagger}^{\prime}\right)^{\prime}\right)=\Lambda\left(\left(\beta^{\prime}, \sigma_{\dagger}^{\prime}\right)^{\prime}\right)
\end{aligned}
$$

It follows that $\sigma$ can be set to any value in $\Theta_{\sigma}$ for simulation purposes with no bearing on estimation nor inference via objective functions of the form

$$
S(X ; \vartheta)=(\hat{\lambda}-\Lambda(\vartheta))^{\prime}(\hat{\lambda}-\Lambda(\vartheta))
$$

In line with the above cited literature, (1.2.10) uses an identity weighting matrix. Other choices are possible as long as $S(X ; \vartheta)$ remains invariant to $\sigma$, or that the distribution of $S(X ; \vartheta)$ does not depend on $\sigma$ under the null hypothesis that fixes $\beta$. The latter pivotality assumption does not require numerical invariance to $\sigma$ throughout the considered parameter space. Instead, a - possibly restricted - estimate of $\sigma$ may be available that evacuates the parameter only under the null. While Assump- 
tion 1 suffices for the ARMA models we consider below, we formalize the alternative pivotality assumption for completion as follows.

Assumption 2. In the context of model (1.2.1) and the null hypothesis

$$
H_{0}\left(\beta_{0}\right): \beta=\beta_{0}, \quad \beta_{0} \text { known }
$$

an objective function $\left.S\left(X ;\left(\beta^{\prime}, \hat{\sigma}^{\prime}\right)^{\prime}\right)\right)$, where $\hat{\sigma}$ is a conformable estimate of $\sigma$, is invariant to $\sigma$ if the null distribution of $\left.S\left(X ;\left(\beta_{0}^{\prime}, \hat{\sigma}^{\prime}\right)^{\prime}\right)\right)$ does not depend on $\sigma$.

A common special case includes scale invariance which implies robustness to multiplying the data by any positive scalar $a$, so Assumptions 1 yields:

$$
\begin{aligned}
x_{h}\left(\left(\beta^{\prime}, a\right)^{\prime}\right) & =a x_{h}\left(\left(\beta^{\prime}, 1\right)^{\prime}\right) \\
\hat{\Lambda}\left(x_{h}\left(\left(\beta^{\prime}, 1\right)^{\prime}\right)\right) & =\hat{\Lambda}\left(x_{h}\left(\left(\beta^{\prime}, a\right)^{\prime}\right)\right), \\
\hat{\Lambda}(\boldsymbol{a} X)) & =\hat{\Lambda}(X) .
\end{aligned}
$$

Its multivariate counterpart involves multiplying the data by an invertible matrix. For illustrative finite sample cases, see Beaulieu, Dufour and Khalaf $(2007,2013)$ and Dufour, Khalaf and Beaulieu (2003, 2010). In these papers, the inverted tests for multivariate Student-t and mixtures of normals distributions require Assumption 2, and the estimate in question uses the sum-of-squares residuals matrix.

To simplify notation in what follows, since $\sigma$ is partialled-out, that is, will be set in simulations to any known value in it parameter space, we suppress it out in the notation for $S(X ; \vartheta), \Lambda(\vartheta), \hat{\lambda}_{\boldsymbol{h}}(\vartheta)$ and $x_{h}(\vartheta)$, which we will redefine as $S(X ; \beta), \Lambda(\beta)$, $\hat{\lambda}_{\boldsymbol{h}}(\beta)$ and $x_{h}(\beta)$. 


\subsection{Inference via test inversion}

Standard applications of Indirect Inference resort to minimizing (1.2.10) and formulating adequate regularity conditions so that the resulting estimator, denoted $\hat{\beta}$, is (typically) asymptotically normal with a covariance matrix that can be estimated consistently. To compare and contrast our proposed inference method with common practices and to define test inversion within a familiar setting, let us revisit the traditional $\alpha$-level confidence interval

$$
C I(g(\beta) ; \alpha)=\left\{g(\hat{\beta}) \pm z_{\alpha / 2} \hat{\Sigma}_{g}^{1 / 2}\right\}
$$

for a given scalar function $g(\beta)$, where $\hat{\Sigma}_{g}^{1 / 2}$ denotes the relevant standard error estimate computed (for example, via the delta-method) from the estimated asymptotic variance/covariance matrix of $\hat{\beta}$, and $z_{\alpha / 2}$ is two-tailed standard normal critical point. As is well known, $C I(g(\beta) ; \alpha)$ is derived by solving, over $g\left(\beta_{0}\right)$, the following inequality

$$
\left|g(\hat{\beta})-g\left(\beta_{0}\right)\right| / \hat{\Sigma}_{g}^{1 / 2} \leq z_{\alpha / 2}
$$

Said differently, the solution of (1.3.1) inverts a t-type test of

$$
H_{g}: g(\beta)=g\left(\beta_{0}\right), \quad \beta_{0} \text { known }
$$

based on the statistic $|g(\hat{\beta})-g(\beta)| / \hat{\Sigma}_{g}^{1 / 2}$.

Ideally, $\mathrm{P}[g(\beta) \in C I(g(\beta) ; \alpha)] \geq 1-\alpha$, at least as $T \rightarrow \infty$. However, identification failures and the above cited pile up effects, root cancellations or stationarity concerns will cause intervals of this form to severely under-cover, even with large samples. In our research, we depart from this approach and propose a confidence set for $\beta$ based on inverting a test of

$$
H_{0}\left(\beta_{0}\right): \beta=\beta_{0}, \quad \beta_{0} \text { known }
$$


using (1.2.10) which we treat as a test statistic. Inverting a test of $H_{0}\left(\beta_{0}\right)$ at a given level $\alpha$ consists in collecting, numerically or analytically, the $\beta_{0}$ values that are not rejected using the considered test at the considered level. For example, given the right-tailed test statistic

$$
\mathcal{T}\left(\beta_{0}\right)=S\left(X, \beta_{0}\right)=\left(\hat{\lambda}-\Lambda\left(\beta_{0}\right)\right)^{\prime}\left(\hat{\lambda}-\Lambda\left(\beta_{0}\right)\right)
$$

and assuming (for the moment and for illustrative purposes) that an $\alpha$-level cut-off point $\mathcal{T}_{c}$ is available, test inversion involves solving, over $\beta_{0}$, the inequality $\mathcal{T}\left(\beta_{0}\right)<$ $\mathcal{T}_{c}$. The solution of this inequality is a parameter space subset, denoted $C S(\beta ; \alpha)$, that satisfies

$$
\mathrm{P}[\beta \in C S(\beta ; \alpha)] \geq 1-\alpha
$$

Since the finite sample distribution of (1.2.10) is intractable, and since there is no reason to expect that a cut-off point that is invariant to $\beta_{0}$ can be found, a numerical solution is required.

We propose to collect by numerical search, the $\beta_{0}$ values that are not rejected using a MC $p$-value that we will define in the next section, denoted $\hat{p}\left(\beta_{0}\right)$. In other words, we propose to search for the $\beta_{0}$ values for which $\hat{p}\left(\beta_{0}\right)>\alpha$. The output of such a search is a joint $\alpha$-level confidence region, which we denote $C S(\beta ; \alpha)$ as above. The set thus derived can be empty, which will provide a diagnostic test of the hypothesized model. Said differently, an empty confidence set would occur when all values of $\beta$ are rejected at the considered level: in this case, the considered specification would be incompatible with available data. Our proposed variant of Indirect Inference thus preserves its specification-robustness attributes. If identification is weak, the set will be unbounded or diffuse, which will also provide useful information regarding model fit (or lack thereof).

If a confidence interval for the individual components of $\beta$ or, more generally, for 
a given function $g(\beta)$ is desired, the above defined $C S(\beta ; \alpha)$ can be projected. This implies minimizing and maximizing $g(\beta)$ over the $\beta$ values in $C S(\beta ; \alpha)$. The ARMAbased impulse-response special case we study below illustrates the usefulness of such projections.

\subsubsection{Exact $p$-values}

We propose applying Dufour's (2006) MC method to compute the above $\hat{p}\left(\beta_{0}\right)$ empirical $p$-value. Given that the null distribution of the considered statistic is nuisance parameter free, the MC method controls type I error even if $S\left(X, \beta_{0}\right)$ is itself simulation-based, in finite samples. The method can be summarized as follows.

We introduce a new layer of simulation, for each value of $\beta_{0}$ : we draw $L$ replications satisfying the null hypothesis from the model, regardless the choice for $\sigma$

$$
x_{\boldsymbol{l}}\left(\beta_{0}\right)=\left(x_{l, 1}\left(\beta_{0}\right), \ldots, x_{l, t}\left(\beta_{0}\right), \ldots, x_{l, T}\left(\beta_{0}\right)\right)^{\prime}, \quad l=1 \ldots L .
$$

These replications should be generated independently from the simulations underlying $S\left(X, \beta_{0}\right)$. Next, we apply $\hat{\Lambda}($.$) to each replication l$ leading to:

$$
\tilde{\lambda}_{l}\left(\beta_{0}\right)=\hat{\Lambda}\left(x_{l}\left(\beta_{0}\right)\right), \quad l=1, \ldots, L,
$$

and compute a series of statistics similar to the one in (1.3.4), but now considering the vector of the estimated parameters $\tilde{\lambda}_{l}\left(\beta_{0}\right)$ for each replication $l$

$$
S_{l}\left(x_{\boldsymbol{l}}\left(\beta_{0}\right)\right)=\left(\tilde{\lambda}_{\boldsymbol{l}}\left(\beta_{0}\right)-\Lambda\left(\beta_{0}\right)\right)^{\prime}\left(\tilde{\lambda}_{\boldsymbol{l}}\left(\beta_{0}\right)-\Lambda\left(\beta_{0}\right)\right)
$$

instead of the vector obtained by applying the estimation method to the data. We

are able to use the same vector of calibrated parameters $\Lambda\left(\beta_{0}\right)$ from (1.3.4) due to the exchangeability property of the MC test [Dufour (2006)]. Lastly, the empirical 
p-value associated with the null hypothesis is computed as:

$$
\hat{p}\left(\beta_{0}\right)=\frac{G_{L}+1}{L+1}
$$

where $G_{L}$ denotes the number of times the statistic $S_{l}\left(x_{\boldsymbol{l}}\left(\beta_{0}\right)\right)$ is greater than or equal to the data based $S\left(X, \beta_{0}\right)$.

\subsection{ARMA special case}

To set focus, we consider the zero mean Gaussian $\operatorname{ARMA}(1,1)$ process of size $T$ with unknown parameters $\theta$ and $\psi$,

$$
X_{t}=\psi X_{t-1}+e_{t}+\theta e_{t-1}, \quad t=1 \ldots T
$$

with

$$
e_{t}=\sigma u_{t}
$$

where $|\theta|<1$ guarantees invertibility or an Autoregressive $\operatorname{AR}(\infty)$ representation, $|\psi|<1$ ensures stationarity, and the joint distribution of $u_{1}, \ldots, u_{t}, \ldots, u_{T}$ is known. For example, $u_{t}$ can be assumed independent and identically distributed with a standard normal distribution:

$$
u_{t} \stackrel{i . i . d .}{\sim} \mathcal{N}(0,1)
$$

We assume that $X_{0}=e_{0}$ where $e_{0}$ is fixed. Student-t errors are also considered in our Monte Carlo study; the generalized lambda family of distributions as considered in Gospodinov and Ng (2015) is another interesting parametrization. Non-zero mean and exogenous variables can be projected out. With regards to the above notation, we maintain $\sigma$ as the nuisance parameter and we focus on joint estimation of $\beta=(\theta, \psi)^{\prime}$. 
In this context, for any positive scalar $a$, we have:

$$
\begin{gathered}
\min _{\gamma} Q(X)=\min _{\gamma} Q(a X)=\operatorname{amin}_{\gamma} Q_{p}(X) \\
\gamma=\left(\gamma_{f}^{\prime}, \gamma_{b}^{\prime}\right)^{\prime}, \quad \gamma_{f}=\left(\gamma_{f 1}, \ldots, \gamma_{f p}\right)^{\prime}, \quad \gamma_{b}=\left(\gamma_{b 1}, \ldots, \gamma_{b p}\right)^{\prime} \\
Q(X)=\sum_{t=p+1}^{T}\left[X_{t}-\gamma_{f 1} X_{t+1}-\ldots-\gamma_{f p} X_{t+p}-\gamma_{b 1} X_{t-1}-\ldots-\gamma_{b p} X_{t-p}\right]^{2},
\end{gathered}
$$

and

$$
\hat{\rho}_{j}=\frac{\widehat{\operatorname{Cov}}\left(X_{t}, X_{t-j}\right)}{\widehat{\operatorname{Var}}\left(X_{t}\right)}=\frac{\widehat{\operatorname{Cov}}\left(a X_{t}, a X_{t-j}\right)}{\widehat{\operatorname{Var}}\left(a X_{t}\right)}, \quad j=1 \ldots p .
$$

These invariance properties guarantees that Indirect Inference on $\beta$ based on $Q($. or on a set of empirical autocorrelations $\hat{\rho}_{\boldsymbol{j}}$ does not require specifying $\sigma$. To simplify notation, we use the same number of leads and lags in $Q(X)$ although clearly, invariance to $\sigma$ does not require this restriction.

Conformably, we consider three choices for our estimating function $\hat{\Lambda}($.$) : (i) two$ -sided OLS estimation with forward and backward-looking components; (ii) OLS estimation of backward-looking or long-AR; and (iii) empirical autocorrelations. For clarity, and given any sample vector $X=\left(X_{1}, \ldots, X_{T}\right)^{\prime}$ of size $T$, we will refer to these choices as

$$
\begin{aligned}
\hat{\Lambda}_{f b}(X) & =\operatorname{ARGMIN} N_{\gamma} Q(X), \text { with } \gamma=\left(\gamma_{f}^{\prime}, \gamma_{b}^{\prime}\right)^{\prime} \\
\hat{\Lambda}_{b}(X) & =A R G M I N_{\gamma_{0}} Q(X), \text { with } \gamma_{0}=\left(0, \gamma_{b}^{\prime}\right)^{\prime}, \\
\hat{\Lambda}_{c}(X) & =\left(\hat{\rho}_{1}, \ldots, \hat{\rho}_{p}\right)^{\prime}
\end{aligned}
$$

where $Q(X)$ corresponds to (1.4.6) and $\hat{\rho}_{j}$ is given by (1.4.7). In each case, to compute the simulation-based counterparts of each for any given value of $\beta$, it suffices to create $H$ simulated paths $x_{h}(\beta)=\left(x_{h, 1}, \ldots, x_{h, t}, \ldots, x_{h, T}\right)^{\prime}$, as

$$
x_{h, t}(\beta)=\psi x_{h, t-1}+u_{h, t}+\theta u_{h, t-1}, \quad t=1 \ldots T, \quad h=1 \ldots H .
$$


The auxiliary parameter estimator for each path using all above estimating functions will numerically coincide with its counterpart based on

$$
\sigma x_{h, t}(\beta)=\psi \sigma x_{h, t-1}+\sigma u_{h, t}+\theta \sigma u_{h, t-1}, \quad t=1 \ldots T, \quad h=1 \ldots H,
$$

for any positive $\sigma$.

Population autocorrelations need not be derived by simulation; yet invariance is explicitly imposed this way for any $p$. For the MA(1) special case, we also use a simplified auxiliary parameter: we fit a long-AR to observed and simulated data and

retain the coefficient of the first lag $X_{t-1}$ as $\hat{\lambda}$. We denote the underlying estimating function as $\Lambda_{*}($.$) .$

\subsubsection{Impulse-response confidence bands}

Impulse-response functions are the most empirically and policy relevant transformations of ARMA parameters. If the stationarity condition is satisfied, (1.4.1) admits an MA $(\infty)$ approximation using lags operators $L$,

$$
\begin{aligned}
X_{t} & =(1-\psi L)^{-1}(1+\theta L) e_{t} \\
& =(1-\phi(L)) e_{t}=\sum_{s=0}^{\infty} \phi^{s} e_{t-s}, \quad t=1 \ldots T
\end{aligned}
$$

and the empirical impulse-response coefficients can be computed following the wellknown iterative process:

$$
\begin{aligned}
& \phi_{0}=1 \\
& \phi_{s}=\psi^{s-1}(\psi+\theta) \text { for } s \geq 1 .
\end{aligned}
$$


These relations define a series of known functions of the vector $\beta=(\theta, \psi)$, denoted $g_{i}(\beta), i=1, \ldots, m$, that can be projected in turn. This implies minimizing and maximizing $g_{i}(\beta)$ over the $\beta$ values in $C S(\beta ; \alpha)$.

Confidence intervals so obtained are simultaneous, in the following sense: let $g_{i}(C S(\beta ; \alpha))$ denote the image of $C S(\beta ; \alpha)$ by the function $g_{i}$. Then (1.3.5) implies that

$$
\mathrm{P}\left[g_{i}(\beta) \in g_{i}(C S(\beta ; \alpha)), \quad i=1, \ldots, m\right] \geq 1-\alpha
$$

In this context, our method is related to the research by Jordá (2009), Jordá and Marcellino (2010) and Jordá, Knuppel and Marcellino (2013) on simultaneous confidence bands and applications to forecasting. The simulation study reported below includes an illustrative analysis of such projections emphasizing close to boundary values of $\theta$ and $\psi$ as well as robustness to root cancelation.

\subsection{Simulation Study}

We conduct MC simulation experiments to asses the performance of our method for $\mathrm{MA}(1)$ and $\operatorname{ARMA}(1,1)$ processes. Our objective is to study power and size when the MA parameters are close to the non-invertibility region for both processes, and the AR parameter is close to the non-stationary region for the ARMA. Our experiments are designed as follows. We simulate Gaussian processes and processes where the errors are drawn from a Student- t distribution with 5 degrees of freedom. Simulated samples with number of observations $T=50,100$ and 200, used here as pseudo-data, are drawn from the ARMA model in (11), setting $\sigma=1$ in line with in Assumptions 1 and 2. These sample size choices correspond to common samples selected in MC studies as well as data used in empirical applications.

Each pair of $\{M A, A R\}$ parameters used to generate $\operatorname{ARMA}(1,1)$ processes is denoted in our experiments as $\left\{\theta_{0}, \psi_{0}\right\}$. The outcomes from all our simulation studies 
are available upon request. Here we focus on the following set of pairs to illustrate our results:

$$
\left\{\theta_{0}, \psi_{0}\right\}=\{\{0,0.6\} ;\{0.6,0\} ;\{0.99,0\}\{0.5,0.96\} ;\{0.65,-0.65\} ;\{0.96,0.5\} ;\{0.99,0.99\}\} .
$$

In particular, we set the $\mathrm{AR}$ parameter $\psi=0$ in (1.4.1) a priory to draw samples from MA(1) processes with the values $\left\{\theta_{0}\right\}=\{0.6 ; 0.99\}$.

We studied power under various combinations of the initial settings of our model. We changed the number of parameters from 1 to 3 and 8 for the MA and from 6 to 8 and 12 for the ARMA, taking into consideration the recommendations from Ghysels, Khalaf and Vodounou (2002) and Galbraith and Zinde-Walsh (1994) for the long-AR estimation, and Gorodnichenko et al. (2012) for the estimation with autocorrelations in our experiments.

Since we found that 8 provides the highest power for all the sample sizes, we suggest that for empirical practices, the total number of auxiliary parameters is set to 8 on each of the choices of the estimating function explained in section 2.2: (i) $\gamma_{f}=\gamma_{b}=4$ in the OLS estimation with forward and backward-looking components, (ii) $\gamma=8$ in the OLS estimation of the long-AR, and (iii) $j=8$ in the estimation with empirical autocorrelations. In the case of the simplified auxiliary parameter estimation for the MA process, the long-AR is estimated as (ii), but only the first coefficient is retained.

In all cases, we set the number of simulated paths to $H=3$. We tested $H=1$ and 3 as suggested by Ghysels, Khalaf and Vodounou (2002) for the MA, and also $H=5$ to examine whether it could improve power for ARMA. Then, we selected $H=3$, keeping the number of auxiliary parameters equal to 8 , as the value which provides the highest power for all the sample sizes.

The number of replications in the application of Dufour's (2006) MC method to compute the $p$-values is set to $L=199$, and $N=1000$ replications are considered for the MC simulations. Lastly, an identity weighting matrix is used to preserve the invariance to scale of the statistic associated to every choice of the estimating function.

We report power in terms of empirical rejections and our main results are summarized along the following lines. The results of the experiments conducted for MA(1) processes are presented in Tables 1.1 and 1.2, and the studies related to the $\operatorname{ARMA}(1,1)$ process 
are shown in Tables 1.3 to 1.7. We use abbreviations for each estimating function: OLSFBLC, OLS-long AR and AutoCorr correspond to the OLS estimation with forward and backward-looking components, the OLS estimation of the long-AR and the estimation with empirical autocorrelations, respectively. Size is controlled with our method for all sample sizes, choices of the estimating function, and for both Gaussian and Student- t errors, irrespective of how close the true parameters are to the unit root. It is well known that MLE gives size distortions when the sample size is small, for both, individual parameter tests and joint tests. Size seems to improve for the MLE when the sample size is increased and the parameters are far from the unit root regions. However, when the parameters approach the unit root regions, size fails for the MLE as the sample size increases, which confirms its asymptotic failure. ${ }^{4}$

The results obtained in term of power for processes with Student- $t$ errors are in line with the ones obtained with Gaussian errors. This outcome confirms the robustness to fatter tails, since we purposely ignore the fact that the errors are drawn from the Studentt distribution when we apply our method.

From the experiments for the MA processes, in Tables 1.1 and 1.2, we can identify that the pile up effect starts for values of the parameter higher than 0.6 for finite samples. Although our method does not produce size distortions, it cannot discriminate between tested values higher than 0.85 when the true value is between 0.85 and 1 . For small values of the parameter, the method with the simplified auxiliary parameter estimation inspired in Galbraith and Zinde-Walsh $(1994,1997)$ shows an increase in power with respect to the OLS estimation of the long-AR. However, as the value of the parameter increases and gets closer to the unit root region, the latter method shows higher power than the former. This result indicates that overidentification is useful to increase power when we have higher persistence in MA(1) processes.

In general, the power of our method increases for the three estimating functions applied to the ARMA as the sample size increases, but none of them steadily shows the highest power for all sample sizes and pairs tested. In Table 1.3, the true pair $\left\{\theta_{0}, \psi_{0}\right\}=\{0,0.6\}$

\footnotetext{
${ }^{4}$ This is noteworthy in view of the above cited problems discussed in the literature.
} 
is equivalent to an $\operatorname{AR}(1)$ process with high persistence. In this case, the method with the first choice of estimating function, the OLS estimation with forward and backward-looking components, shows the highest power for most of the pairs tested, including the almost unit root pair $\left\{\theta^{*}, \psi^{*}\right\}=\{0.99,0.99\}$ for small sample sizes, $T=50$ and 100. As we increase the sample size to $T=200$, the power obtained with our method for each of the three choices of estimating function increases and reaches similar values.

In Tables 1.4 and 1.5 we have two pairs with one parameter closer to the unit root than the other. In Table 1.4, for the true pair $\left\{\theta_{0}, \psi_{0}\right\}=\{0.5,0.96\}$, using the autocorrelations as the estimating function in our method gives more power for most of the pairs tested when the sample size is $T=50$ and 100 . However, its power is very poor for the almost unit root pair; and as a result, the method with the first choice of estimating function, the OLS estimation with FBLC, is the one able to discriminate this pair with the highest power. As before, once we increase the sample size, power reaches similar values when our method is applied with the three estimating functions.

In Table 1.5, for the true pair $\left\{\theta_{0}, \psi_{0}\right\}=\{0.96,0.5\}$, the second choice of the estimating function, the OLS estimation of the long-AR, shows the highest power for most of the pairs tested when the sample size is $T=50$ and 100. Still, choosing the autocorrelations as the estimating function enables to discriminate between the true pair and the almost unit root pair, since power is poor when we use the other two estimating functions, for this particular pair. When the sample size is $T=200$, power increases for the three estimating functions, yet we need the autocorrelations to ensure we can discriminate the almost unit root pair.

The results obtained with the root cancelation pair, $\left\{\theta_{0}, \psi_{0}\right\}=\{0.65,-0.65\}$ are reported in Table 1.6. The top section of this table compares our method verus MLE in terms of size. Note MLE is oversize while for our method size remains exact. The method applied with the OLS estimation with FBLC gives the highest power for almost all the pairs tested when the sample size is $T=50$. Once we increase the sample size to $T=100$, this estimating functions still shows the highest power followed closely by the second choice of estimating function, the OLS estimation of the long-AR. When the sample size is $T=200$, the power obtained with the autocorrelations as estimating function reaches closely to the 
power obtained with the other two estimating functions.

Lastly, in Table 1.7 we examine the almost unit root pair as the true pair, $\left\{\theta_{0}, \psi_{0}\right\}=$ $\{0.99,0.99\}$. For most of the pairs tested, applying the method with the OLS estimation of the long-AR gives the highest power when the sample size is $T=50$ and 100 . However, for these sample sizes, using autocorrelations gives the highest power for the last three pairs tested, for which the value of the MA parameter is closer to the boundary than the value of the AR parameter. As we increase the sample size, power reaches similar values for our method with the three estimating functions.

\subsubsection{Robustness checks}

In addition to our previous experiments, we conducted simple robustness checks for our method. Two examples are shown in Tables 1.8 and 1.9. We present the power values for two ARMA processes which are misspecified as $\operatorname{ARMA}(1,1)$ with a sample size $T=100$. In Table 1.8 we have an $\operatorname{ARMA}(2,1)$ with $\left\{\theta_{0}, \psi_{1,0}, \psi_{2,0}\right\}=\{0.3,0.6,0.85\}$, so we have a high persistence $\operatorname{AR}(2)$ mixed with a low persistence MA(1). In general, the method applied with autocorrelations offers more power rejecting the pairs where the AR parameter is closer to the boundary than the MA parameter, while with the OLS estimation with forward and backward-looking components we obtain more power rejecting the pairs where the MA parameter is closer to the boundary than the AR parameter.

The ARMA $(2,1)$ in Table 1.9 with $\left\{\theta_{1,0}, \theta_{2,0}, \psi_{0}\right\}=\{0.3,0.85,0.6\}$, shows a high persistence $\mathrm{AR}(1)$ mixed with a $\mathrm{MA}(2)$ which has higher persistence on its second parameter. In this case, we find that the method applied with both, the OLS estimation of forward and backward-looking components and the OLS estimation of the long-AR, is more powerful rejecting the misspecification than with the autocorrelations. Note that in both examples, our three choices of estimating functions provide good power, rejecting the misspecification for all the pairs tested. 


\subsubsection{Impulse-response confidence bands}

To illustrate the transformation via the computation of impulse-response confidence bands we conduct further simulation exercises. We simulate one ARMA(1,1) sample path selecting different combinations of the parameters $\left\{\theta_{0}, \psi_{0}\right\}$. The setting of our model is the same that we followed on the above mentioned experiments: the total number of auxiliary parameters is set to 8 on the three choices for the estimating function, $H=3$ and $L=199$. We conduct a grid search on the four quadrants with a step of 0.01 .

Figures 1.1 and 1.2 show two simulations with the pairs $\left\{\theta_{0}, \psi_{0}\right\}=\{0.99,0.99\}$ and $\left\{\theta_{0}, \psi_{0}\right\}=\{0.96,0.5\}$ for samples of $T=50$ observations. Each figure contains four diagrams: the simulated path is plotted on the first one, the joint $95 \%$ confidence set appears on the second one, the joint $95 \%$ confidence surface is on the third one, and the impulse-response confidence bands, jointly with MLE impulse-response function, are shown on the fourth one. These figures present the outcomes of our method selecting the OLS estimation with forward and backward-looking components (FBLC) and the autocorrelations, respectively as estimating functions.

Even with this small sample size, the true values of the parameters and the resulting impulse-response are covered by our $95 \%$ confidence set and impulse-response confidence bands, respectively. However, for the almost unit root pair in Figure 1.1, the impulseresponse function obtained from the MLE is outside the confidence bands. As expected, inverting the asymptotic Wald test based on MLE estimates is almost infeasible at corner solutions with parameters close to the unit boundary, since in many instances the variancecovariance matrix is not invertible. Comparing our impulse-response confidence bands with the impulse-response function obtained with the MLE point estimates, we conclude that the MLE can lead to spurious results when identification problems exist.

Additionally, Figure 1.3 illustrates the robustness of our method to root cancelation for a path of $T=50$ observations constructed with the pair $\left\{\theta_{0}, \psi_{0}\right\}=\{0.65,-0.65\}$ and considering the FBLC estimating function. In this particular case, MLE point estimates are incorrectly identified due to flat segments in the objective function resulting in confidence sets which do not cover the true values of the coefficients. In contrast, our confidence sets 
cover the true values of the parameters and, as a result, the impulse-response confidence bands are able to capture the true impulse-response function.

\subsection{Conclusions}

In this chapter we revisit Indirect Inference jointly with the MC test method from a confidence set two-stage simulation-based outlook focusing on ARMA processes in finite samples. Despite the simplicity of ARMA models in time series, identification and boundary issues raise enduring complications for estimation and inference. We propose estimating equations via two sided regressions with forward and backward-looking components, backward looking autoregressions and autocorrelations. We treat the resulting objective functions as test statistics which we invert through a two-stage simulation to obtain a joint confidence set for the parameters: first, we obtain calibrated estimates applying the previously mentioned estimating functions, then we compute the $p$-values applying MC test methods. Invariance principles are proposed to ensure finite sample exactness in general nuisance parameter dependent settings. The proposed impulse-responses confidence bands represent an important and empirically relevant transformation of the joint confidence set.

Supportive simulation results confirm the following. In contrast to MLE-based tests, our proposed methods achieve size control and good power, irrespective of how close the parameters are to the unit root boundary. The results obtained in term of power for processes with Student-t errors are in line with the ones obtained with Gaussian errors. Our method eradicates pile up problems even with very small samples, for example, with no more than 50 observations. The significance level is affected neither by flat segments in the objective function, nor by how close the estimated parameters are to the unit root boundary. Wide confidence sets allow for root cancelations; however, this result does not translate into a widening of our impulse-response confidence bands. Finally, our studies on impulse-response confidence bands shows that relying on MLE when the processes are highly persistent can lead researches to underestimate shock effects when conducting estimation and forecasting. 


\section{References}

[1] Andrews D. W. K. and P. Guggenberger (2010). Asymptotic Size and a Problem with Subsampling and With the $m$ out of $n$ Bootstrap. Econometric Theory 26, 426-468.

[2] Andrews, D. W.K. and Xu Cheng (2012). Estimation and Inference with Weak, SemiStrong and Strong-Identification. Econometrica 80, 2153-2211.

[3] Andrews, Isaiah and A. Mikusheva (2014). Weak Identification in Maximum Likelihood: A Question of Information. American Economics Review 104, 195-99.

[4] Andrews, Isaiah and A. Mikusheva (2015). Maximum Likelihood Inference in Weakly Identified Dynamic Stochastic General Equilibrium Models. Quantitative Economics $1,123-152$.

[5] Ansley, C.F.and Newbold, P. (1980). Finite Sample Properties of Estimators for Autoregressive Moving Average Processes. Journal of Econometrics 13, 159-184.

[6] Barnard G. A. (1963). Comment on The Spectral Analysis of Point Processes by M.S. Bartlett. Journal of the Royal Statistical Society, Series B 25, 294.

[7] Beaulieu M.-C., Dufour J.-M. and L. Khalaf (2007). Multivariate Tests of MeanVariance Efficiency with Possibly Non-Gaussian Errors: An Exact Simulation-Based Approach. Journal of Business and Economic Statistics 25, 398-410.

[8] Beaulieu M.-C., Dufour J.-M. and L. Khalaf (2013). Identification-Robust Estimation and Testing of the Zero-Beta CAPM. Review of Economic Studies 80, 892-924. 
[9] Beaulieu M-C., Dufour J.-M. and L. Khalaf (2014). Exact Confidence Set Estimation and Goodness of Fit Test Methods for Asymmetric Heavy Tailed Stable Distributions. Journal of Econometrics 181, 3-14.

[10] Billio, Monica and Alain Monfort (2003). Kernel-Based Indirect Inference. Journal of Financial Econometrics 3, 297-326.

[11] Bolduc D., L. Khalaf, and C.Yélou (2010). Identification Robust Confidence Set Methods for Inference on Parameter Ratios with Application to Discrete Choice Models. Journal of Econometrics 157, 317-327.

[12] Calvet L. E. and V. Czellar (2015). Through the Looking Glass: Indirect Inference via Simple Equilibria. Journal of Econometrics 185, 343-358.

[13] Calzolari, G. , G. Fionretini and E. Sentana (2004). Constrained Indirect Estimation The Review of Economic Studies Limited, 71 945-973.

[14] Chaudhuri, S., David T. Frazier and Eric Renault (2015). Indirect Inference with Endogenously Missing Exogenous Variables. Working paper.

[15] Davis R. and W. Dunsmuir (1996). Maximum Likelihood Estimation for MA(1) processes with a Root on the Unit Circle. Econometric Theory 12, 1-20.

[16] Davis R. and L. Song (2011). Unit Roots in Moving Averages Beyond First Order. Annals of Statistics 39, 3062-3091.

[17] Dominicy Y. and D. Veredas (2013). The Method of Simulated Quantiles. Journal of Econometrics 172, 235-247.

[18] Dufour J.-M, L. Khalaf and M.-C. Beaulieu (2003). Exact Skewness and Kurtosis Tests for Multivariate Normality and Goodness of Fit in Multivariate Regressions with Application to Asset Pricing Models, Oxford Bulletin of Economics and Statistics 65, 891-906. 
[19] Dufour. J.-M, L. Khalaf and M.-C. Beaulieu (2010). Finite Sample Diagnostics in Multivariate Regressions with Applications to Asset Pricing Models, Journal of Applied Econometrics 25, 263-285.

[20] Dufour J.-M. and J. R. Kurz-Kim (2010). Exact Inference and Optimal Invariant Estimation for the Stability Parameter of Symmetric $\alpha$-Stable Distributions. Journal of Empirical Finance 17, 180-194.

[21] Dufour J.-M. (2006). Monte Carlo Tests with Nuisance Parameters: A General Approach to Finite-Sample Inference and Nonstandard Asymptotics in Econometrics. Journal of Econometrics 133, 443-478.

[22] Dufour J.-M. and D. Pelletier (2014). Practical Methods for Modelling Weak VARMA Processes: Identification, Estimation and Specification with a Macroeconomic Application. Working paper.

[23] Dufour J.-M. and O. Torrès (2000). Markovian Processes, Two-Sided Autoregressions and Exact Inference for Stationary and Nonstationary Autoregressive Processes. Journal of Econometrics 99, 255-289.

[24] Dufour J.-M. and P. Valéry (2006). On a Simple Two-Stage Closed-Form Estimator for a Stochastic Volatility in a General Linear Regression; in Volume 20 (Part A) of Advances in Econometrics: Econometric Analysis of Economic and Financial Time Series, in honor of Clive Granger and Robert Engle, edited by Thomas B. Fomby and Dek Terrell, Elsevier Science, Oxford, UK, 259-288.

[25] Dufour J.-M. and P. Valéry (2009). Exact and Assymptotic Test for Possibly NonRegular Hypothesis on Stochastic Volatility Models. Journal of Econometrics 150, 193-206.

[26] Dridi R., Renault E. and A. Guay (2007). Indirect Inference and Calibration of Dynamic Stochastic General Equilibrium Models. Journal of Econometrics 136, 397-430. 
[27] Dwass M. (1957). Modified Randomization Test for Nonparametric Hypothesis. Annals of Mathematical Statistics 28, 181-187.

[28] Forneron, Jean-Jacques and Senera Ng (2015). The ABC of Simulation Estimation with Auxiliary Statistics. Working paper.

[29] Fuleky P. and E. Zivot (2014). Indirect Inference Based on the Score. Econometrics Journal 17, 383-393.

[30] Galbraith J. W. and V. Zinde-Walsh (1994). A Simple, Non-Iterative Estimator for Moving Average Models. Biometrika 81, 143-156.

[31] Galbraith J. W. and V. Zinde-Walsh (1997). On Simple Auto-Regressive-Based Estimation and Identification Techniques for ARMA Models. Biometrika 84, 685-696.

[32] Gallant A. R. and G. Tauchen (1996). Which Moments to Match. Econometric Theory $12,657-681$.

[33] Genton, M.G. and Ronchetti, E. (2003). Journal of the American Statistical Association $98,67-76$.

[34] Ghysels E., Khalaf L. and C. Vodounou (2003). Simulation Based Inference in Moving Average Models. Annales D'Economie et Statistique 69, 85-99.

[35] Gorodnichenko Y., Mikusheva A. and S. Ng (2012). Estimators for Persistent and Possibly Non-Stationary Data with Classical Properties. Econometric Theory 28, 10031036.

[36] Gospodinov N. (2002). Bootstrap Based Inference in Models with a Nearly Noninvertible Moving Average Component. Journal of Business and Economic Statistics 20, $254-268$.

[37] Gospodinov N. and S. Ng (2015). Minimum Distance Estimation of Possibly NonInvertible Moving Average Models. Journal of Business and Economic Statistics 33, 403-417. 
[38] Gouriéroux C., Monfort A. and E. Renault (1993). Indirect Inference. Journal of Applied Econometrics 8, S85-S118.

[39] Gouriéroux C. and A. Monfort (1996). Simulation -Based Econometric Methods, Core Lectures, Oxford University Press.

[40] Gouriéroux C., Phillips P. C. B. and J. Yu (2010). Indirect Inference for Dynamic Panel Models. Journal of Econometrics 157, 68-77.

[41] Guay A. and O. Scaillet (2003). Indirect Inference, Nuisance Parameter, and Threshold Moving Average Models. Journal of Business and Economic Statistics 21, 122-132.

[42] Hannan E. J. and J. R. Rissanen (1982). Recursive Estimation of Mixed AutoregressiveMoving-Average Order. Biometrika 69, 81-94.

[43] Hansen B. E. (1999). The Grid Bootstrap and the Autoregressive Model. Review of Economics and Statistics 81, 594-607.

[44] Harvey (1981) Time Series models, Philip Allan (London).

[45] Jordá O. (2009). Simultaneous Confidence Regions for Impulse Responses The Review of Economics and Statistics 91, 629-647.

[46] Jordá O. and M. Marcellino (2010). Path Forecast Evaluation. Journal of Applied Econometrics 25, 635-662.

[47] Jordá O. and S. Kozicki (2011). Estimation and Inference by the Method of Projection Minimum Distance: An Application to the New Keynesian Hybrid Phillips Curve. International Economic Review 52, 461-487.

[48] Jordá O., Knuppel M. and M. Marcellino (2013). Empirical Simultaneous Confidence Regions for Path-Forecasts. International Journal of Forecasting 29, 456-468.

[49] Li T. (2010). Indirect Inference in Structural Econometric Models. Journal of Econometrics 157, 120-128.

[50] MATLAB 8.1, The MathWorks, Inc., Natick, Massachusetts, United States. 
[51] Mikusheva A. (2007). Uniform Inference in Autoregressive Models. Econometrica 75, $1411-1452$

[52] Mikusheva A. (2012). One-Dimensional Inference in Autoregressive Models with the Potential Presence of a Unit Root. Econometrica 80, 173-212.

[53] Mikusheva A. (2014). Second Order Expansion of the t-statistic in AR(1) Models. Econometric Theory 31, 426- 448.

[54] Nelson, C.R. and R. Startz( 2007). The Zero-information-limit Condition and Spurious Inference in Weakly-identified Models. Journal of Econometrics 138, 47-62.

[55] Phillips P. C. B. (2014). On Confidence Intervals for Autoregressive Roots and Predictive Rgressions. Econometrica 82, 1177-1195.

[56] Robins, J. M., Aad Van der Vaart and Valérie Ventura (2000). Journal of the American Statistical Association 95, 1143- 1156.

[57] Ronchetti, E. and Fabio Trojani (2001). Robust Inference with GMM Estimators. Journal of Econometrics 101, 37-69.

[58] Sargan J. D. and A. Bhargava (1983). Maximum Likelihood Estimation of Regression Models with First Order Moving Average Errors When the Root Lies on the Unit Circle. Econometrica 51, 799-820.

[59] Smith A. (1993). Estimating Nonlinear Time Series Models Using Simulated Vector Autoregressions. Journal of Applied Econometrics 8, S63-S84.

[60] Stock J. H., and M. W. Watson (1993). A Simple Estimator of Cointegrating Vectors in Higher Order Integrated Systems. Econometrica 61, 783-820. 
Table 1.1: Empirical Rejections: True value $\theta_{0}=0.6$

\section{POWER}

\begin{tabular}{|c|c|c|c|c|}
\hline $\mathrm{T}=50$ & \multicolumn{2}{|c|}{ Gaussian MA } & \multicolumn{2}{c|}{ Student-t $(\mathrm{df}=5)$ MA } \\
\hline Tested value & OLS-long AR & Simplified & OLS-long AR & Simplified \\
\hline$\{0.00\}$ & 0.717 & 0.861 & 0.720 & 0.865 \\
\hline$\{0.30\}$ & 0.322 & 0.364 & 0.302 & 0.370 \\
\hline$\{0.85\}$ & 0.276 & 0.225 & 0.266 & 0.211 \\
\hline$\{0.90\}$ & 0.368 & 0.286 & 0.371 & 0.267 \\
\hline$\{0.96\}$ & 0.436 & 0.324 & 0.430 & 0.302 \\
\hline$\{0.99\}$ & 0.442 & 0.334 & 0.442 & 0.309 \\
\hline
\end{tabular}

\begin{tabular}{|c|c|c|c|c|}
\hline $\mathrm{T}=100$ & \multicolumn{2}{|c|}{ Gaussian MA } & \multicolumn{2}{c|}{ Student-t $(\mathrm{df}=5) \mathrm{MA}$} \\
\hline Tested value & OLS-long AR & Simplified & OLS-long AR & Simplified \\
\hline$\{0.00\}$ & 0.988 & 0.994 & 0.989 & 0.995 \\
\hline$\{0.30\}$ & 0.628 & 0.699 & 0.634 & 0.703 \\
\hline$\{0.85\}$ & 0.611 & 0.476 & 0.623 & 0.470 \\
\hline$\{0.90\}$ & 0.721 & 0.569 & 0.729 & 0.558 \\
\hline$\{0.96\}$ & 0.785 & 0.634 & 0.796 & 0.610 \\
\hline$\{0.99\}$ & 0.790 & 0.640 & 0.806 & 0.618 \\
\hline
\end{tabular}

\begin{tabular}{|c|c|c|c|c|}
\hline $\mathrm{T}=200$ & \multicolumn{2}{|c|}{ Gaussian MA } & \multicolumn{2}{c|}{ Student-t $(\mathrm{df}=5)$ MA } \\
\hline Tested value & OLS-long AR & Simplified & OLS-long AR & Simplified \\
\hline$\{0.00\}$ & 1.000 & 1.000 & 1.000 & 1.000 \\
\hline$\{0.30\}$ & 0.926 & 0.935 & 0.934 & 0.942 \\
\hline$\{0.85\}$ & 0.910 & 0.786 & 0.910 & 0.779 \\
\hline$\{0.90\}$ & 0.960 & 0.869 & 0.967 & 0.862 \\
\hline$\{0.96\}$ & 0.978 & 0.914 & 0.983 & 0.908 \\
\hline$\{0.99\}$ & 0.980 & 0.919 & 0.984 & 0.912 \\
\hline
\end{tabular}


Table 1.2: Empirical Rejections: True value $\theta_{0}=0.99$

POWER

\begin{tabular}{|c|c|c|c|c|}
\hline $\mathrm{T}=50$ & \multicolumn{2}{|c|}{ Gaussian MA } & \multicolumn{2}{c|}{ Student-t $(\mathrm{df}=5)$ MA } \\
\hline Tested value & OLS-long AR & Simplified & OLS-long AR & Simplified \\
\hline$\{0.00\}$ & 0.998 & 0.993 & 0.998 & 0.993 \\
\hline$\{0.30\}$ & 0.948 & 0.854 & 0.961 & 0.842 \\
\hline$\{0.60\}$ & 0.594 & 0.363 & 0.573 & 0.353 \\
\hline$\{0.85\}$ & 0.095 & 0.073 & 0.091 & 0.061 \\
\hline$\{0.90\}$ & 0.061 & 0.054 & 0.059 & 0.049 \\
\hline$\{0.96\}$ & 0.057 & 0.045 & 0.051 & 0.041 \\
\hline
\end{tabular}

\begin{tabular}{|c|c|c|c|c|}
\hline $\mathrm{T}=100$ & \multicolumn{2}{|c|}{ Gaussian MA } & \multicolumn{2}{c|}{ Student-t $(\mathrm{df}=5)$ MA } \\
\hline Tested value & OLS-long AR & Simplified & OLS-long AR & Simplified \\
\hline$\{0.00\}$ & 1.000 & 1.000 & 1.000 & 1.000 \\
\hline$\{0.30\}$ & 1.000 & 0.990 & 1.000 & 0.992 \\
\hline$\{0.60\}$ & 0.918 & 0.676 & 0.916 & 0.690 \\
\hline$\{0.85\}$ & 0.141 & 0.094 & 0.130 & 0.078 \\
\hline$\{0.90\}$ & 0.087 & 0.060 & 0.071 & 0.059 \\
\hline$\{0.96\}$ & 0.059 & 0.053 & 0.045 & 0.040 \\
\hline
\end{tabular}

\begin{tabular}{|c|c|c|c|c|}
\hline $\mathrm{T}=200$ & \multicolumn{2}{|c|}{ Gaussian MA } & \multicolumn{2}{c|}{ Student-t $(\mathrm{df}=5) \mathrm{MA}$} \\
\hline Tested value & OLS-long AR & Simplified & OLS-long AR & Simplified \\
\hline$\{0.00\}$ & 1.000 & 1.000 & 1.000 & 1.000 \\
\hline$\{0.30\}$ & 1.000 & 1.000 & 1.000 & 1.000 \\
\hline$\{0.60\}$ & 0.996 & 0.922 & 0.996 & 0.928 \\
\hline$\{0.85\}$ & 0.204 & 0.099 & 0.196 & 0.124 \\
\hline$\{0.90\}$ & 0.084 & 0.050 & 0.079 & 0.063 \\
\hline$\{0.96\}$ & 0.042 & 0.039 & 0.043 & 0.040 \\
\hline
\end{tabular}


Table 1.3: Empirical Rejections: True pair $\left\{\theta_{0}, \psi_{0}\right\}=\{0,0.6\}$

POWER

\begin{tabular}{|c|c|c|c|c|c|c|}
\hline $\mathrm{T}=50$ & \multicolumn{3}{|c|}{ Gaussian ARMA } & \multicolumn{3}{c|}{ Student-t (df=5) ARMA } \\
\hline Tested pair & OLS-FBLC & OLS-long AR & AutoCorr & OLS-FBLC & OLS-long AR & AutoCorr \\
\hline$\{0.3,0.2\}$ & 0.075 & 0.078 & 0.249 & 0.064 & 0.071 & 0.252 \\
\hline$\{0.3,0.85\}$ & 0.157 & 0.111 & 0.284 & 0.157 & 0.104 & 0.282 \\
\hline$\{0.5,0.5\}$ & 0.463 & 0.188 & 0.035 & 0.443 & 0.178 & 0.031 \\
\hline$\{0.5,0.96\}$ & 0.547 & 0.388 & 0.757 & 0.545 & 0.377 & 0.752 \\
\hline$\{0.6,0\}$ & 0.353 & 0.159 & 0.305 & 0.351 & 0.145 & 0.312 \\
\hline$\{0.6,0.85\}$ & 0.760 & 0.519 & 0.294 & 0.760 & 0.524 & 0.303 \\
\hline$\{0.85,0.2\}$ & 0.959 & 0.726 & 0.138 & 0.967 & 0.721 & 0.131 \\
\hline$\{0.85,0.6\}$ & 0.979 & 0.834 & 0.032 & 0.986 & 0.846 & 0.024 \\
\hline$\{0.96,0.5\}$ & 0.988 & 0.871 & 0.031 & 0.990 & 0.871 & 0.030 \\
\hline$\{0.99,0.99\}$ & 0.990 & 0.925 & 0.884 & 0.992 & 0.937 & 0.894 \\
\hline
\end{tabular}

\begin{tabular}{|c|c|c|c|c|c|c|}
\hline T=100 & \multicolumn{3}{|c|}{ Gaussian ARMA } & \multicolumn{3}{c|}{ Student-t (df=5) ARMA } \\
\hline Tested pair & OLS-FBLC & OLS-long AR & AutoCorr & OLS-FBLC & OLS-long AR & AutoCorr \\
\hline$\{0.3,0.2\}$ & 0.109 & 0.104 & 0.392 & 0.097 & 0.091 & 0.384 \\
\hline$\{0.3,0.85\}$ & 0.323 & 0.487 & 0.612 & 0.311 & 0.478 & 0.600 \\
\hline$\{0.5,0.5\}$ & 0.738 & 0.589 & 0.055 & 0.734 & 0.580 & 0.047 \\
\hline$\{0.5,0.96\}$ & 0.820 & 0.904 & 0.981 & 0.827 & 0.911 & 0.987 \\
\hline$\{0.6,0\}$ & 0.655 & 0.399 & 0.511 & 0.639 & 0.393 & 0.501 \\
\hline$\{0.6,0.85\}$ & 0.959 & 0.949 & 0.636 & 0.963 & 0.951 & 0.626 \\
\hline$\{0.85,0.2\}$ & 1.000 & 0.980 & 0.221 & 0.998 & 0.983 & 0.205 \\
\hline$\{0.85,0.6\}$ & 1.000 & 0.995 & 0.053 & 1.000 & 0.996 & 0.047 \\
\hline$\{0.96,0.5\}$ & 1.000 & 0.998 & 0.052 & 1.000 & 0.998 & 0.044 \\
\hline$\{0.99,0.99\}$ & 1.000 & 1.000 & 0.995 & 1.000 & 1.000 & 0.997 \\
\hline
\end{tabular}

\begin{tabular}{|c|c|c|c|c|c|c|}
\hline $\mathrm{T}=200$ & \multicolumn{3}{|c|}{ Gaussian ARMA } & \multicolumn{3}{c|}{ Student-t (df=5) ARMA } \\
\hline Tested pair & OLS-FBLC & OLS-long AR & AutoCorr & OLS-FBLC & OLS-long AR & AutoCorr \\
\hline$\{0.3,0.2\}$ & 0.157 & 0.198 & 0.630 & 0.177 & 0.198 & 0.636 \\
\hline$\{0.3,0.85\}$ & 0.551 & 0.943 & 0.903 & 0.561 & 0.940 & 0.899 \\
\hline$\{0.5,0.5\}$ & 0.948 & 0.942 & 0.039 & 0.955 & 0.943 & 0.056 \\
\hline$\{0.5,0.96\}$ & 0.973 & 0.999 & 0.999 & 0.976 & 0.999 & 0.999 \\
\hline$\{0.6,0\}$ & 0.888 & 0.759 & 0.795 & 0.889 & 0.761 & 0.805 \\
\hline$\{0.6,0.85\}$ & 0.997 & 0.999 & 0.911 & 0.997 & 1.000 & 0.913 \\
\hline$\{0.85,0.2\}$ & 1.000 & 1.000 & 0.387 & 1.000 & 1.000 & 0.381 \\
\hline$\{0.85,0.6\}$ & 1.000 & 1.000 & 0.089 & 1.000 & 1.000 & 0.076 \\
\hline$\{0.96,0.5\}$ & 1.000 & 1.000 & 0.047 & 1.000 & 1.000 & 0.055 \\
\hline$\{0.99,0.99\}$ & 1.000 & 1.000 & 1.000 & 1.000 & 1.000 & 1.000 \\
\hline
\end{tabular}


Table 1.4: Empirical Rejections: True pair $\left\{\theta_{0}, \psi_{0}\right\}=\{0.5,0.96\}$

\section{POWER}

\begin{tabular}{|c|c|c|c|c|c|c|}
\hline T $=50$ & \multicolumn{3}{|c|}{ Gaussian ARMA } & \multicolumn{3}{c|}{ Student-t (df=5) ARMA } \\
\hline Tested pair & OLS-FBLC & OLS-long AR & AutoCorr & OLS-FBLC & OLS-long AR & AutoCorr \\
\hline$\{0,0.6\}$ & 0.394 & 0.922 & 0.859 & 0.389 & 0.916 & 0.861 \\
\hline$\{0.3,0.2\}$ & 0.227 & 0.940 & 0.985 & 0.233 & 0.938 & 0.988 \\
\hline$\{0.3,0.85\}$ & 0.087 & 0.241 & 0.207 & 0.087 & 0.225 & 0.220 \\
\hline$\{0.5,0.5\}$ & 0.040 & 0.223 & 0.845 & 0.034 & 0.223 & 0.850 \\
\hline$\{0.6,0\}$ & 0.057 & 0.785 & 0.991 & 0.049 & 0.776 & 0.990 \\
\hline$\{0.6,0.85\}$ & 0.100 & 0.058 & 0.195 & 0.098 & 0.045 & 0.209 \\
\hline$\{0.85,0.2\}$ & 0.499 & 0.319 & 0.958 & 0.489 & 0.308 & 0.958 \\
\hline$\{0.85,0.6\}$ & 0.617 & 0.341 & 0.729 & 0.605 & 0.334 & 0.734 \\
\hline$\{0.96,0.5\}$ & 0.717 & 0.442 & 0.823 & 0.719 & 0.423 & 0.832 \\
\hline$\{0.99,0.99\}$ & 0.735 & 0.584 & 0.084 & 0.745 & 0.578 & 0.077 \\
\hline
\end{tabular}

\begin{tabular}{|c|c|c|c|c|c|c|}
\hline T=100 & \multicolumn{3}{|c|}{ Gaussian ARMA } & \multicolumn{3}{c|}{ Student-t (df=5) ARMA } \\
\hline Tested pair & OLS-FBLC & OLS-long AR & AutoCorr & OLS-FBLC & OLS-long AR & AutoCorr \\
\hline$\{0,0.6\}$ & 0.766 & 1.000 & 0.986 & 0.764 & 1.000 & 0.985 \\
\hline$\{0.3,0.2\}$ & 0.540 & 1.000 & 1.000 & 0.531 & 1.000 & 1.000 \\
\hline$\{0.3,0.85\}$ & 0.175 & 0.428 & 0.403 & 0.183 & 0.437 & 0.404 \\
\hline$\{0.5,0.5\}$ & 0.037 & 0.441 & 0.985 & 0.030 & 0.423 & 0.981 \\
\hline$\{0.6,0\}$ & 0.080 & 0.996 & 1.000 & 0.072 & 0.993 & 1.000 \\
\hline$\{0.6,0.85\}$ & 0.157 & 0.075 & 0.383 & 0.152 & 0.071 & 0.376 \\
\hline$\{0.85,0.2\}$ & 0.802 & 0.636 & 0.999 & 0.789 & 0.627 & 0.999 \\
\hline$\{0.85,0.6\}$ & 0.869 & 0.681 & 0.935 & 0.868 & 0.672 & 0.931 \\
\hline$\{0.96,0.5\}$ & 0.932 & 0.778 & 0.976 & 0.939 & 0.770 & 0.977 \\
\hline$\{0.99,0.99\}$ & 0.934 & 0.908 & 0.140 & 0.942 & 0.907 & 0.133 \\
\hline
\end{tabular}

\begin{tabular}{|c|c|c|c|c|c|c|}
\hline $\mathrm{T}=200$ & \multicolumn{3}{|c|}{ Gaussian ARMA } & \multicolumn{3}{c|}{ Student-t (df=5) ARMA } \\
\hline Tested pair & OLS-FBLC & OLS-long AR & AutoCorr & OLS-FBLC & OLS-long AR & AutoCorr \\
\hline$\{0,0.6\}$ & 0.976 & 1.000 & 1.000 & 0.975 & 1.000 & 0.999 \\
\hline$\{0.3,0.2\}$ & 0.877 & 1.000 & 1.000 & 0.871 & 1.000 & 1.000 \\
\hline$\{0.3,0.85\}$ & 0.371 & 0.775 & 0.713 & 0.366 & 0.773 & 0.714 \\
\hline$\{0.5,0.5\}$ & 0.054 & 0.786 & 1.000 & 0.052 & 0.802 & 0.999 \\
\hline$\{0.6,0\}$ & 0.194 & 1.000 & 1.000 & 0.203 & 1.000 & 1.000 \\
\hline$\{0.6,0.85\}$ & 0.222 & 0.136 & 0.689 & 0.228 & 0.132 & 0.696 \\
\hline$\{0.85,0.2\}$ & 0.970 & 0.918 & 1.000 & 0.966 & 0.922 & 1.000 \\
\hline$\{0.85,0.6\}$ & 0.982 & 0.911 & 0.998 & 0.986 & 0.919 & 0.999 \\
\hline$\{0.96,0.5\}$ & 0.997 & 0.958 & 1.000 & 0.997 & 0.959 & 0.999 \\
\hline$\{0.99,0.99\}$ & 0.997 & 0.995 & 0.271 & 0.997 & 0.997 & 0.258 \\
\hline
\end{tabular}


Table 1.5: Empirical Rejections: True pair $\left\{\theta_{0}, \psi_{0}\right\}=\{0.96,0.5\}$

\section{POWER}

\begin{tabular}{|c|c|c|c|c|c|c|}
\hline T $=50$ & \multicolumn{3}{|c|}{ Gaussian ARMA } & \multicolumn{3}{c|}{ Student-t (df=5) ARMA } \\
\hline Tested pair & OLS-FBLC & OLS-long AR & AutoCorr & OLS-FBLC & OLS-long AR & AutoCorr \\
\hline$\{0,0.6\}$ & 0.924 & 0.996 & 0.089 & 0.922 & 0.997 & 0.074 \\
\hline$\{0.3,0.2\}$ & 0.829 & 0.995 & 0.363 & 0.838 & 0.996 & 0.365 \\
\hline$\{0.3,0.85\}$ & 0.632 & 0.841 & 0.296 & 0.631 & 0.852 & 0.298 \\
\hline$\{0.5,0.5\}$ & 0.349 & 0.769 & 0.056 & 0.338 & 0.782 & 0.044 \\
\hline$\{0.5,0.96\}$ & 0.339 & 0.449 & 0.758 & 0.338 & 0.450 & 0.746 \\
\hline$\{0.6,0\}$ & 0.454 & 0.961 & 0.431 & 0.467 & 0.962 & 0.438 \\
\hline$\{0.6,0.85\}$ & 0.183 & 0.291 & 0.308 & 0.186 & 0.296 & 0.312 \\
\hline$\{0.85,0.2\}$ & 0.028 & 0.281 & 0.184 & 0.030 & 0.281 & 0.175 \\
\hline$\{0.85,0.6\}$ & 0.033 & 0.051 & 0.049 & 0.038 & 0.043 & 0.040 \\
\hline$\{0.99,0.99\}$ & 0.064 & 0.084 & 0.872 & 0.067 & 0.084 & 0.882 \\
\hline
\end{tabular}

\begin{tabular}{|c|c|c|c|c|c|c|}
\hline T=100 & \multicolumn{3}{|c|}{ Gaussian ARMA } & \multicolumn{3}{c|}{ Student-t (df=5) ARMA } \\
\hline Tested pair & OLS-FBLC & OLS-long AR & AutoCorr & OLS-FBLC & OLS-long AR & AutoCorr \\
\hline$\{0,0.6\}$ & 0.997 & 1.000 & 0.099 & 0.997 & 1.000 & 0.083 \\
\hline$\{0.3,0.2\}$ & 0.980 & 1.000 & 0.556 & 0.984 & 1.000 & 0.551 \\
\hline$\{0.3,0.85\}$ & 0.923 & 0.994 & 0.618 & 0.919 & 0.997 & 0.601 \\
\hline$\{0.5,0.5\}$ & 0.719 & 0.982 & 0.069 & 0.728 & 0.982 & 0.060 \\
\hline$\{0.5,0.96\}$ & 0.699 & 0.826 & 0.979 & 0.718 & 0.839 & 0.986 \\
\hline$\{0.6,0\}$ & 0.829 & 1.000 & 0.658 & 0.827 & 1.000 & 0.661 \\
\hline$\{0.6,0.85\}$ & 0.489 & 0.599 & 0.635 & 0.499 & 0.593 & 0.624 \\
\hline$\{0.85,0.2\}$ & 0.057 & 0.492 & 0.267 & 0.057 & 0.514 & 0.249 \\
\hline$\{0.85,0.6\}$ & 0.063 & 0.063 & 0.068 & 0.070 & 0.073 & 0.057 \\
\hline$\{0.99,0.99\}$ & 0.090 & 0.240 & 0.996 & 0.092 & 0.244 & 0.997 \\
\hline
\end{tabular}

\begin{tabular}{|c|c|c|c|c|c|c|}
\hline $\mathrm{T}=200$ & \multicolumn{3}{|c|}{ Gaussian ARMA } & \multicolumn{3}{c|}{ Student-t (df=5) ARMA } \\
\hline Tested pair & OLS-FBLC & OLS-long AR & AutoCorr & OLS-FBLC & OLS-long AR & AutoCorr \\
\hline$\{0,0.6\}$ & 1.000 & 1.000 & 0.099 & 1.000 & 1.000 & 0.106 \\
\hline$\{0.3,0.2\}$ & 1.000 & 1.000 & 0.843 & 1.000 & 1.000 & 0.845 \\
\hline$\{0.3,0.85\}$ & 0.992 & 1.000 & 0.898 & 0.992 & 1.000 & 0.894 \\
\hline$\{0.5,0.5\}$ & 0.952 & 1.000 & 0.047 & 0.957 & 1.000 & 0.060 \\
\hline$\{0.5,0.96\}$ & 0.945 & 0.989 & 0.999 & 0.947 & 0.985 & 0.999 \\
\hline$\{0.6,0\}$ & 0.986 & 1.000 & 0.925 & 0.986 & 1.000 & 0.928 \\
\hline$\{0.6,0.85\}$ & 0.837 & 0.915 & 0.909 & 0.828 & 0.919 & 0.905 \\
\hline$\{0.85,0.2\}$ & 0.124 & 0.820 & 0.461 & 0.103 & 0.814 & 0.443 \\
\hline$\{0.85,0.6\}$ & 0.113 & 0.080 & 0.085 & 0.099 & 0.084 & 0.083 \\
\hline$\{0.99,0.99\}$ & 0.152 & 0.534 & 1.000 & 0.143 & 0.534 & 1.000 \\
\hline
\end{tabular}


Table 1.6: Empirical Rejections: True pair $\left\{\theta_{0}, \psi_{0}\right\}=\{0.65,-0.65\}$

SIZE

\begin{tabular}{|c|c|c|c|c|c|c|}
\cline { 2 - 7 } \multicolumn{1}{c|}{} & \multicolumn{3}{c|}{ Gaussian ARMA } & \multicolumn{3}{c|}{ Student-t (df=5) ARMA } \\
\hline T & OLS-FBLC & OLS-long AR & AutoCorr & OLS-FBLC & OLS-long AR & AutoCorr \\
\hline 50 & 0.056 & 0.053 & 0.038 & 0.041 & 0.049 & 0.033 \\
\hline 100 & 0.053 & 0.054 & 0.058 & 0.041 & 0.048 & 0.051 \\
\hline 200 & 0.049 & 0.045 & 0.054 & 0.049 & 0.043 & 0.061 \\
\hline
\end{tabular}

\begin{tabular}{|c|c|c|c|c|c|c|}
\hline MLE & \multicolumn{3}{|c|}{ Gaussian ARMA } & \multicolumn{3}{c|}{ Student-t (df=5) ARMA } \\
\hline $\mathrm{T}$ & OLS-FBLC & OLS-long AR & AutoCorr & OLS-FBLC & OLS-long AR & AutoCorr \\
\hline 50 & 0.470 & 0.454 & 0.523 & 0.446 & 0.401 & 0.500 \\
\hline 100 & 0.432 & 0.417 & 0.482 & 0.404 & 0.392 & 0.457 \\
\hline 200 & 0.414 & 0.414 & 0.470 & 0.370 & 0.371 & 0.445 \\
\hline
\end{tabular}

POWER

\begin{tabular}{|c|c|c|c|c|c|c|}
\hline T=50 & \multicolumn{3}{|c|}{ Gaussian ARMA } & \multicolumn{3}{c|}{ Student-t (df=5) ARMA } \\
\hline Tested pair & OLS-FBLC & OLS-long AR & AutoCorr & OLS-FBLC & OLS-long AR & AutoCorr \\
\hline$\{0,0.6\}$ & 0.430 & 0.225 & 0.181 & 0.418 & 0.229 & 0.174 \\
\hline$\{0.3,0.2\}$ & 0.806 & 0.728 & 0.858 & 0.796 & 0.740 & 0.866 \\
\hline$\{0.3,0.85\}$ & 0.923 & 0.699 & 0.453 & 0.932 & 0.702 & 0.428 \\
\hline$\{0.5,0.5\}$ & 0.956 & 0.866 & 0.981 & 0.958 & 0.858 & 0.986 \\
\hline$\{0.6,0\}$ & 0.836 & 0.436 & 0.180 & 0.828 & 0.431 & 0.170 \\
\hline$\{0.6,0.85\}$ & 0.985 & 0.878 & 0.880 & 0.992 & 0.884 & 0.880 \\
\hline$\{0.85,0.2\}$ & 0.999 & 0.916 & 0.307 & 0.999 & 0.904 & 0.271 \\
\hline$\{0.85,0.6\}$ & 1.000 & 0.955 & 0.568 & 1.000 & 0.961 & 0.557 \\
\hline$\{0.96,0.5\}$ & 1.000 & 0.961 & 0.481 & 1.000 & 0.963 & 0.471 \\
\hline$\{0.99,0.99\}$ & 1.000 & 0.980 & 0.997 & 1.000 & 0.979 & 0.998 \\
\hline
\end{tabular}

\begin{tabular}{|c|c|c|c|c|c|c|}
\hline T=100 & \multicolumn{3}{|c|}{ Gaussian ARMA } & \multicolumn{3}{c|}{ Student-t (df=5) ARMA } \\
\hline Tested pair & OLS-FBLC & OLS-long AR & AutoCorr & OLS-FBLC & OLS-long AR & AutoCorr \\
\hline$\{0,0.6\}$ & 0.797 & 0.695 & 0.500 & 0.793 & 0.700 & 0.493 \\
\hline$\{0.3,0.2\}$ & 0.980 & 0.998 & 0.991 & 0.981 & 0.999 & 0.990 \\
\hline$\{0.3,0.85\}$ & 0.996 & 0.995 & 0.875 & 0.996 & 0.994 & 0.870 \\
\hline$\{0.5,0.5\}$ & 0.999 & 1.000 & 0.999 & 1.000 & 1.000 & 0.999 \\
\hline$\{0.6,0\}$ & 0.983 & 0.901 & 0.509 & 0.989 & 0.899 & 0.502 \\
\hline$\{0.6,0.85\}$ & 1.000 & 1.000 & 0.992 & 1.000 & 1.000 & 0.991 \\
\hline$\{0.85,0.2\}$ & 1.000 & 1.000 & 0.727 & 1.000 & 0.999 & 0.727 \\
\hline$\{0.85,0.6\}$ & 1.000 & 1.000 & 0.933 & 1.000 & 1.000 & 0.932 \\
\hline$\{0.96,0.5\}$ & 1.000 & 1.000 & 0.901 & 1.000 & 1.000 & 0.894 \\
\hline$\{0.99,0.99\}$ & 1.000 & 1.000 & 1.000 & 1.000 & 1.000 & 1.000 \\
\hline
\end{tabular}


Table 1.6 contd.: Empirical Rejections: True pair $\left\{\theta_{0}, \psi_{0}\right\}=\{0.65,-0.65\}$

POWER

\begin{tabular}{|c|c|c|c|c|c|c|}
\hline $\mathrm{T}=200$ & \multicolumn{3}{|c|}{ Gaussian ARMA } & \multicolumn{3}{c|}{ Student-t (df=5) ARMA } \\
\hline Tested pair & OLS-FBLC & OLS-long AR & AutoCorr & OLS-FBLC & OLS-long AR & AutoCorr \\
\hline$\{0,0.6\}$ & 0.984 & 0.979 & 0.887 & 0.987 & 0.983 & 0.894 \\
\hline$\{0.3,0.2\}$ & 0.999 & 1.000 & 1.000 & 0.999 & 1.000 & 1.000 \\
\hline$\{0.3,0.85\}$ & 1.000 & 1.000 & 0.998 & 1.000 & 1.000 & 0.996 \\
\hline$\{0.5,0.5\}$ & 1.000 & 1.000 & 1.000 & 1.000 & 1.000 & 1.000 \\
\hline$\{0.6,0\}$ & 0.999 & 0.999 & 0.911 & 1.000 & 0.999 & 0.918 \\
\hline$\{0.6,0.85\}$ & 1.000 & 1.000 & 1.000 & 1.000 & 1.000 & 1.000 \\
\hline$\{0.85,0.2\}$ & 1.000 & 1.000 & 0.994 & 1.000 & 1.000 & 0.992 \\
\hline$\{0.85,0.6\}$ & 1.000 & 1.000 & 1.000 & 1.000 & 1.000 & 0.999 \\
\hline$\{0.96,0.5\}$ & 1.000 & 1.000 & 0.998 & 1.000 & 1.000 & 0.998 \\
\hline$\{0.99,0.99\}$ & 1.000 & 1.000 & 1.000 & 1.000 & 1.000 & 1.000 \\
\hline
\end{tabular}


Table 1.7: Empirical Rejections: True pair $\left\{\theta_{0}, \psi_{0}\right\}=\{0.99,0.99\}$

\section{POWER}

\begin{tabular}{|c|c|c|c|c|c|c|}
\hline $\mathrm{T}=50$ & \multicolumn{3}{|c|}{ Gaussian ARMA } & \multicolumn{3}{c|}{ Student-t (df=5) ARMA } \\
\hline Tested pair & OLS-FBLC & OLS-long AR & AutoCorr & OLS-FBLC & OLS-long AR & AutoCorr \\
\hline$\{0,0.6\}$ & 0.922 & 1.000 & 0.915 & 0.921 & 1.000 & 0.914 \\
\hline$\{0.3,0.2\}$ & 0.831 & 1.000 & 0.992 & 0.830 & 1.000 & 0.991 \\
\hline$\{0.3,0.85\}$ & 0.609 & 0.979 & 0.331 & 0.615 & 0.985 & 0.332 \\
\hline$\{0.5,0.5\}$ & 0.295 & 0.968 & 0.904 & 0.305 & 0.980 & 0.906 \\
\hline$\{0.5,0.96\}$ & 0.285 & 0.778 & 0.046 & 0.290 & 0.794 & 0.046 \\
\hline$\{0.6,0\}$ & 0.427 & 0.998 & 0.998 & 0.442 & 0.999 & 0.992 \\
\hline$\{0.6,0.85\}$ & 0.131 & 0.653 & 0.317 & 0.131 & 0.663 & 0.308 \\
\hline$\{0.85,0.2\}$ & 0.017 & 0.763 & 0.980 & 0.020 & 0.772 & 0.986 \\
\hline$\{0.85,0.6\}$ & 0.023 & 0.304 & 0.815 & 0.024 & 0.293 & 0.825 \\
\hline$\{0.96,0.5\}$ & 0.040 & 0.257 & 0.891 & 0.038 & 0.241 & 0.893 \\
\hline
\end{tabular}

\begin{tabular}{|c|c|c|c|c|c|c|}
\hline $\mathrm{T}=100$ & \multicolumn{3}{|c|}{ Gaussian ARMA } & \multicolumn{3}{c|}{ Student-t (df=5) ARMA } \\
\hline Tested pair & OLS-FBLC & OLS-long AR & AutoCorr & OLS-FBLC & OLS-long AR & AutoCorr \\
\hline$\{0,0.6\}$ & 0.997 & 1.000 & 0.994 & 0.997 & 1.000 & 0.995 \\
\hline$\{0.3,0.2\}$ & 0.980 & 1.000 & 1.000 & 0.984 & 1.000 & 1.000 \\
\hline$\{0.3,0.85\}$ & 0.908 & 1.000 & 0.635 & 0.914 & 1.000 & 0.648 \\
\hline$\{0.5,0.5\}$ & 0.690 & 1.000 & 0.993 & 0.701 & 1.000 & 0.994 \\
\hline$\{0.5,0.96\}$ & 0.662 & 0.984 & 0.074 & 0.671 & 0.988 & 0.070 \\
\hline$\{0.6,0\}$ & 0.826 & 1.000 & 1.000 & 0.824 & 1.000 & 1.000 \\
\hline$\{0.6,0.85\}$ & 0.418 & 0.939 & 0.610 & 0.435 & 0.947 & 0.626 \\
\hline$\{0.85,0.2\}$ & 0.036 & 0.983 & 1.000 & 0.042 & 0.984 & 1.000 \\
\hline$\{0.85,0.6\}$ & 0.035 & 0.508 & 0.976 & 0.033 & 0.531 & 0.973 \\
\hline$\{0.96,0.5\}$ & 0.039 & 0.453 & 0.991 & 0.048 & 0.444 & 0.994 \\
\hline
\end{tabular}

\begin{tabular}{|c|c|c|c|c|c|c|}
\hline $\mathrm{T}=200$ & \multicolumn{3}{|c|}{ Gaussian ARMA } & \multicolumn{3}{c|}{ Student-t (df=5) ARMA } \\
\hline Tested pair & OLS-FBLC & OLS-long AR & AutoCorr & OLS-FBLC & OLS-long AR & AutoCorr \\
\hline$\{0,0.6\}$ & 1.000 & 1.000 & 1.000 & 1.000 & 1.000 & 1.000 \\
\hline$\{0.3,0.2\}$ & 1.000 & 1.000 & 1.000 & 1.000 & 1.000 & 1.000 \\
\hline$\{0.3,0.85\}$ & 0.991 & 1.000 & 0.922 & 0.992 & 1.000 & 0.921 \\
\hline$\{0.5,0.5\}$ & 0.943 & 1.000 & 1.000 & 0.943 & 1.000 & 1.000 \\
\hline$\{0.5,0.96\}$ & 0.932 & 1.000 & 0.157 & 0.935 & 1.000 & 0.163 \\
\hline$\{0.6,0\}$ & 0.984 & 1.000 & 1.000 & 0.984 & 1.000 & 1.000 \\
\hline$\{0.6,0.85\}$ & 0.778 & 0.998 & 0.911 & 0.780 & 0.999 & 0.910 \\
\hline$\{0.85,0.2\}$ & 0.121 & 1.000 & 1.000 & 0.100 & 1.000 & 1.000 \\
\hline$\{0.85,0.6\}$ & 0.052 & 0.840 & 1.000 & 0.034 & 0.833 & 0.999 \\
\hline$\{0.96,0.5\}$ & 0.067 & 0.767 & 1.000 & 0.067 & 0.763 & 1.000 \\
\hline
\end{tabular}


Table 1.8: Empirical Rejections: True values $\left\{\theta_{1,0}, \theta_{2,0}, \psi_{0}\right\}=\{0.3,0.85,0.60\}$

\section{POWER}

\begin{tabular}{|c|c|c|c|}
\hline $\mathrm{T}=100$ & \multicolumn{3}{|c|}{ Gaussian ARMA } \\
\hline Tested pair & OLS-FBLC & OLS-long AR & AutoCorr \\
\hline$\{0,0.6\}$ & 0.903 & 0.998 & 0.326 \\
\hline$\{0.3,0.2\}$ & 0.978 & 0.999 & 0.974 \\
\hline$\{0.3,0.85\}$ & 0.994 & 0.999 & 0.310 \\
\hline$\{0.5,0.5\}$ & 0.998 & 1.000 & 0.275 \\
\hline$\{0.5,0.96\}$ & 1.000 & 1.000 & 0.918 \\
\hline$\{0.6,0\}$ & 0.998 & 0.999 & 0.993 \\
\hline$\{0.6,0.85\}$ & 1.000 & 1.000 & 0.327 \\
\hline$\{0.85,0.2\}$ & 1.000 & 1.000 & 0.862 \\
\hline$\{0.85,0.6\}$ & 1.000 & 1.000 & 0.105 \\
\hline$\{0.96,0.5\}$ & 1.000 & 1.000 & 0.231 \\
\hline$\{0.99,0.99\}$ & 1.000 & 1.000 & 0.987 \\
\hline
\end{tabular}

Table 1.9: Empirical Rejections: True values $\left\{\theta_{0}, \psi_{1,0}, \psi_{2,0}\right\}=\{0.3,0.6,0.85\}$

\section{POWER}

\begin{tabular}{|c|c|c|c|}
\hline $\mathrm{T}=100$ & \multicolumn{3}{|c|}{ Gaussian ARMA } \\
\hline Tested pair & OLS-FBLC & OLS-long AR & AutoCorr \\
\hline$\{0,0.6\}$ & 0.635 & 0.931 & 0.962 \\
\hline$\{0.3,0.2\}$ & 0.403 & 0.957 & 1.000 \\
\hline$\{0.3,0.85\}$ & 0.171 & 0.198 & 0.233 \\
\hline$\{0.5,0.5\}$ & 0.066 & 0.130 & 0.959 \\
\hline$\{0.5,0.96\}$ & 0.105 & 0.117 & 0.103 \\
\hline$\{0.6,0\}$ & 0.056 & 0.733 & 1.000 \\
\hline$\{0.6,0.85\}$ & 0.289 & 0.227 & 0.208 \\
\hline$\{0.85,0.2\}$ & 0.858 & 0.562 & 0.997 \\
\hline$\{0.85,0.6\}$ & 0.930 & 0.814 & 0.898 \\
\hline$\{0.96,0.5\}$ & 0.974 & 0.860 & 0.956 \\
\hline$\{0.99,0.99\}$ & 0.981 & 0.969 & 0.282 \\
\hline
\end{tabular}


Figure 1.1: ARMA $(1,1)$ Simulated Path $\left\{\theta_{0}, \psi_{0}\right\}=\{0.99,0.99\}$

Figure (i)

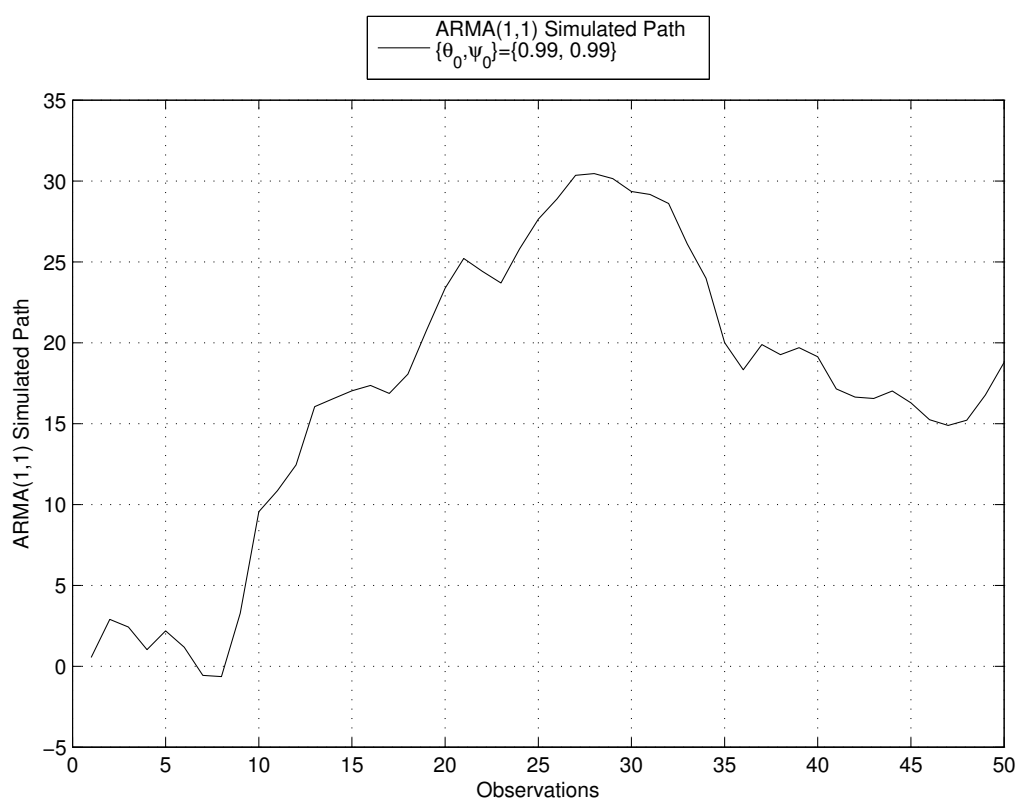

Figure (ii)

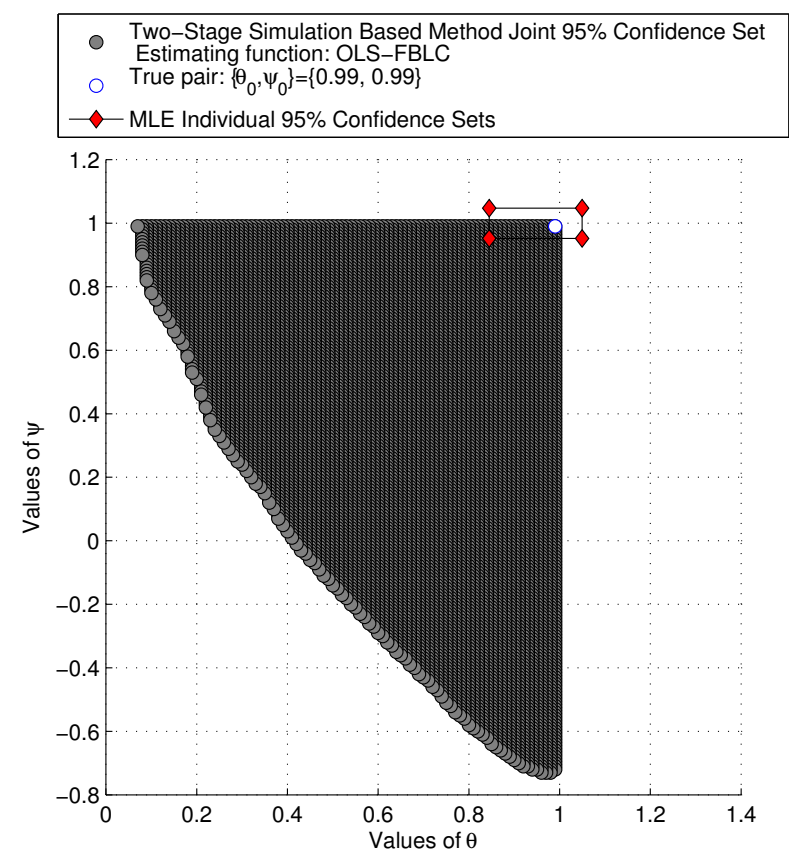


Figure (iii)

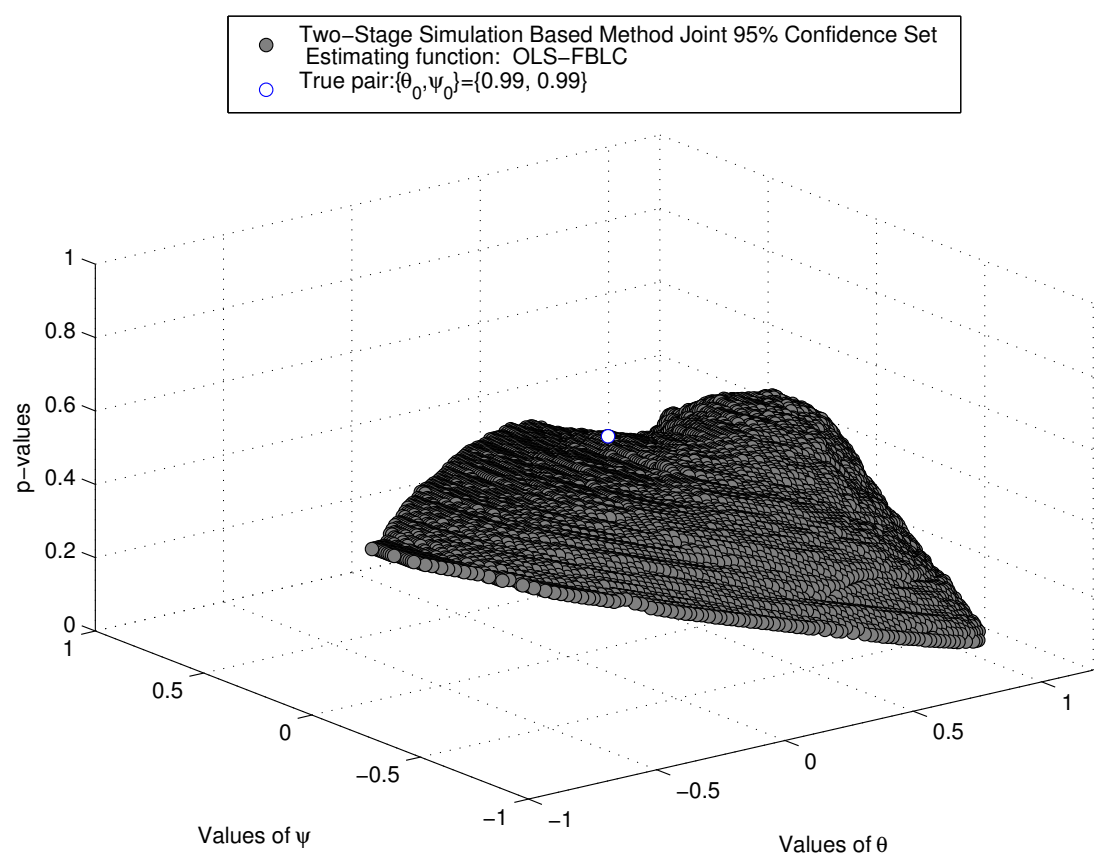

Figure (iv)

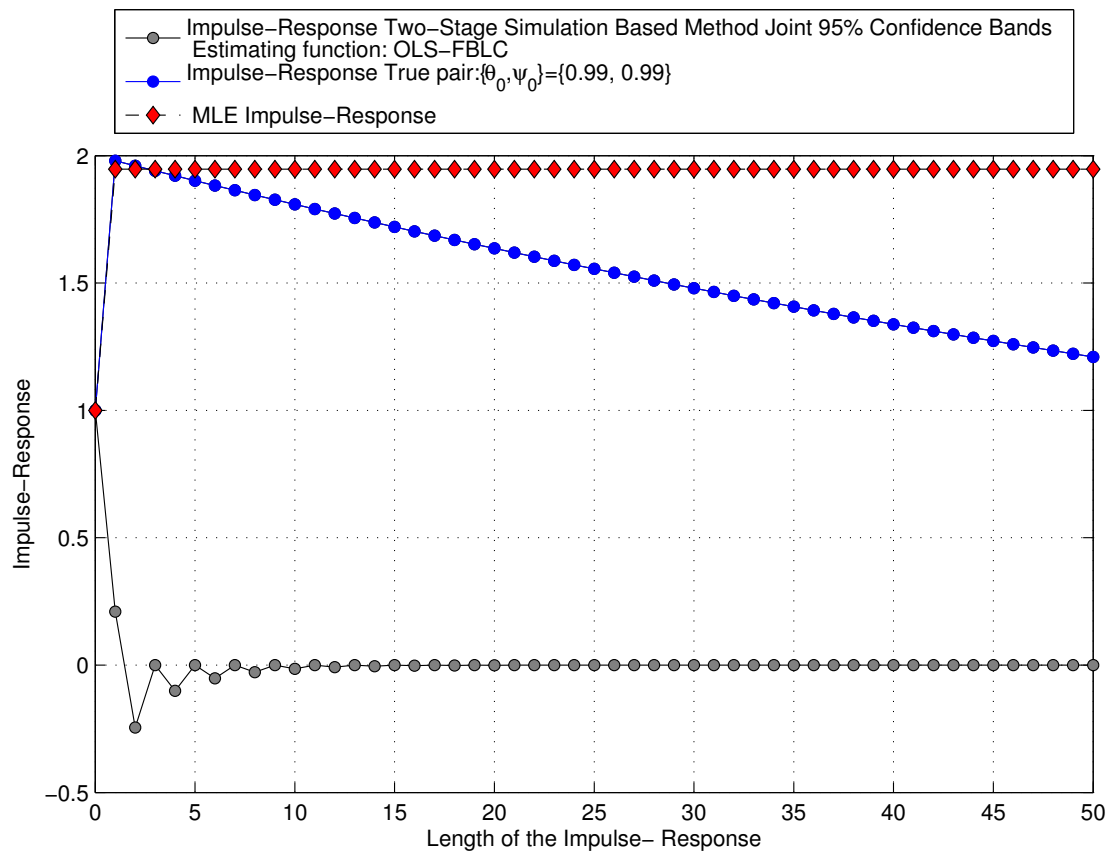


Figure 1.2: $\operatorname{ARMA}(1,1)$ Simulated Path $\left\{\theta_{0}, \psi_{0}\right\}=\{0.96,0.5\}$

Figure (i)

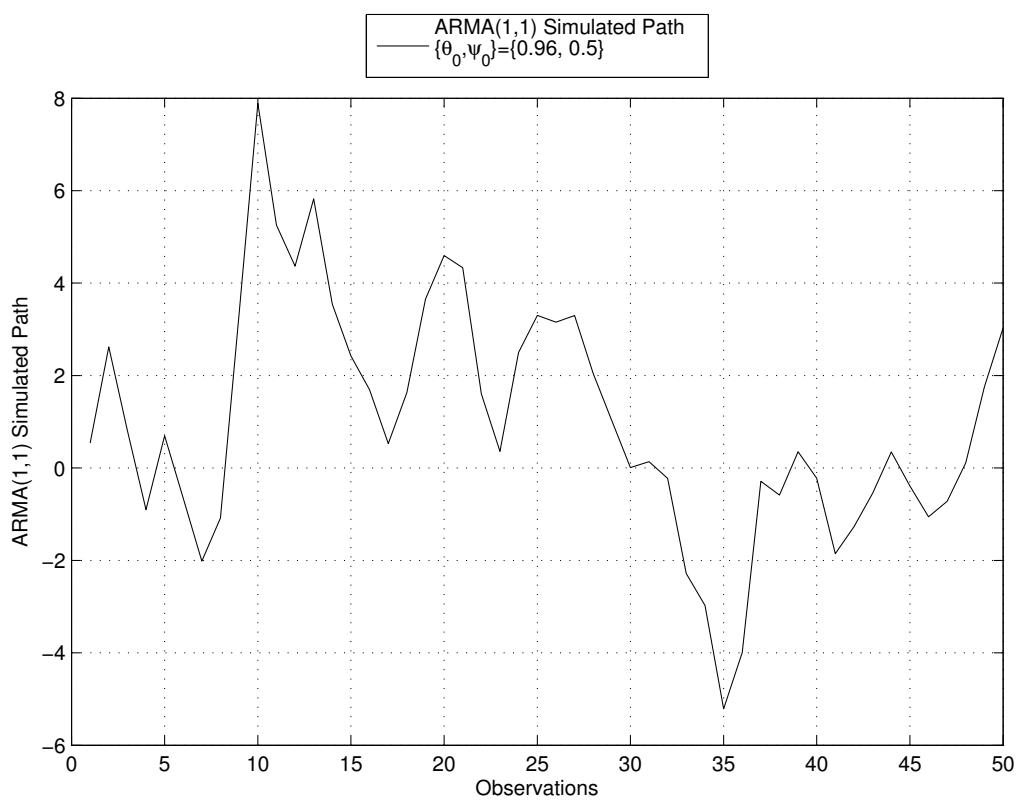

Figure (ii)

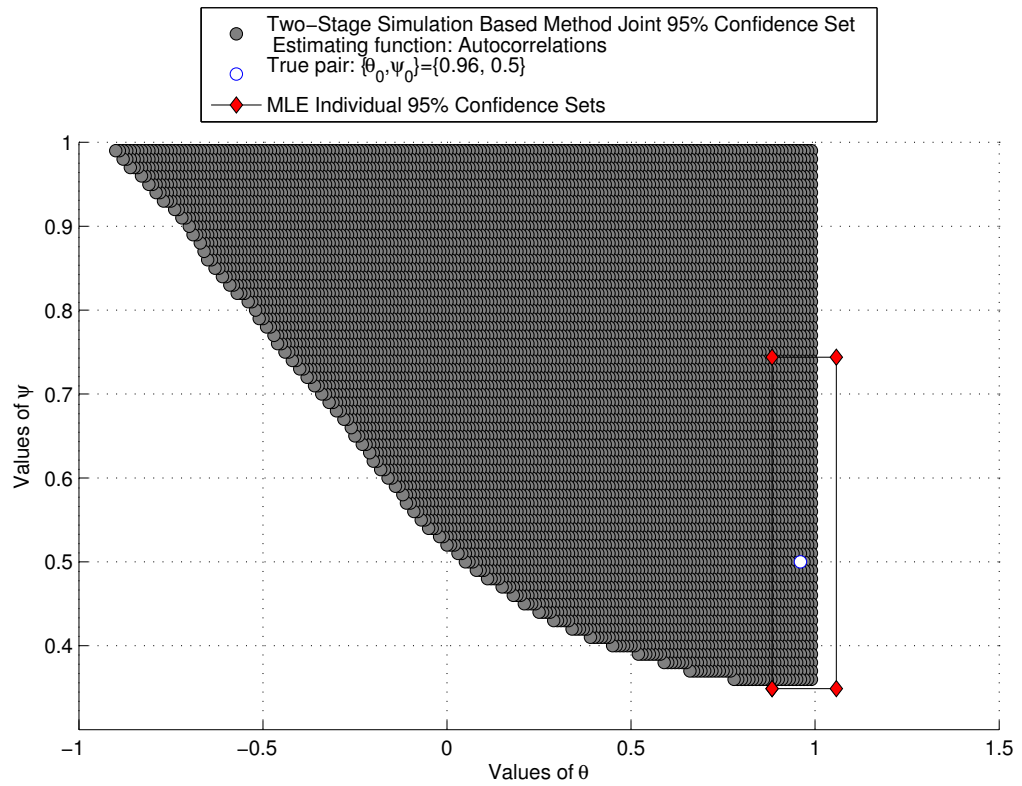


Figure (iii)

- Two-Stage Simulation Based Method Joint 95\% Confidence Set Estimating function: Autocorrelations

- True pair: $\left\{\theta_{0}, \psi_{0}\right\}=\{0.96,0.5\}$

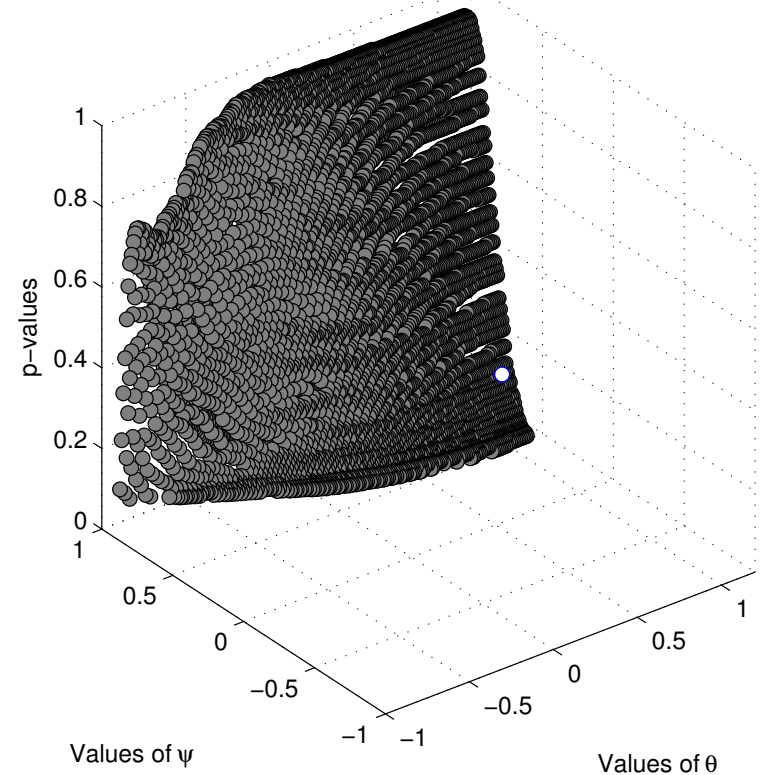

Figure (iv)

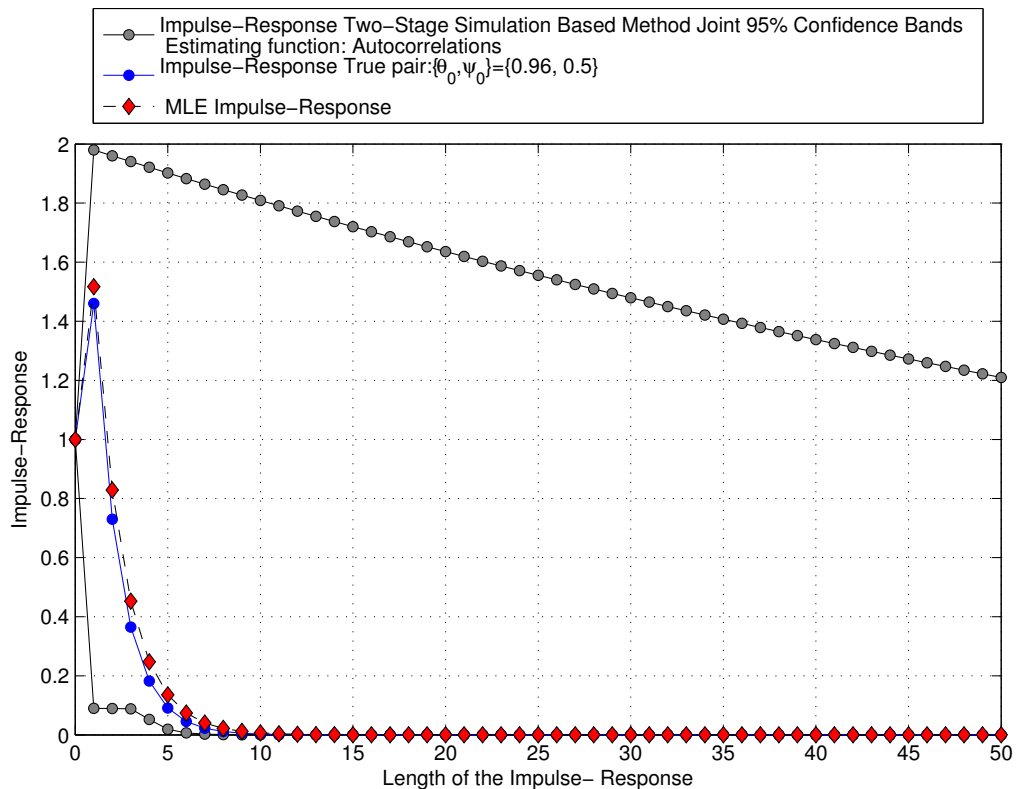


Figure 1.3: $\operatorname{ARMA}(1,1)$ Simulated Path $\left\{\theta_{0}, \psi_{0}\right\}=\{0.65,-0.65\}$

Figure (i)

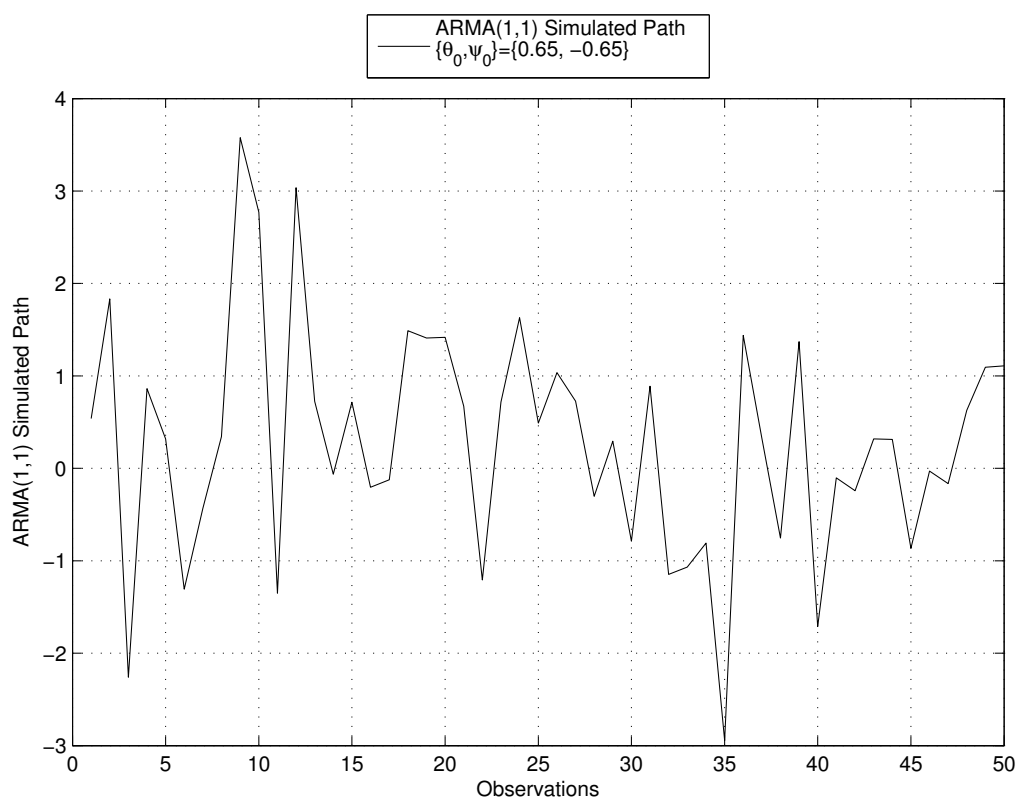

Figure (ii)

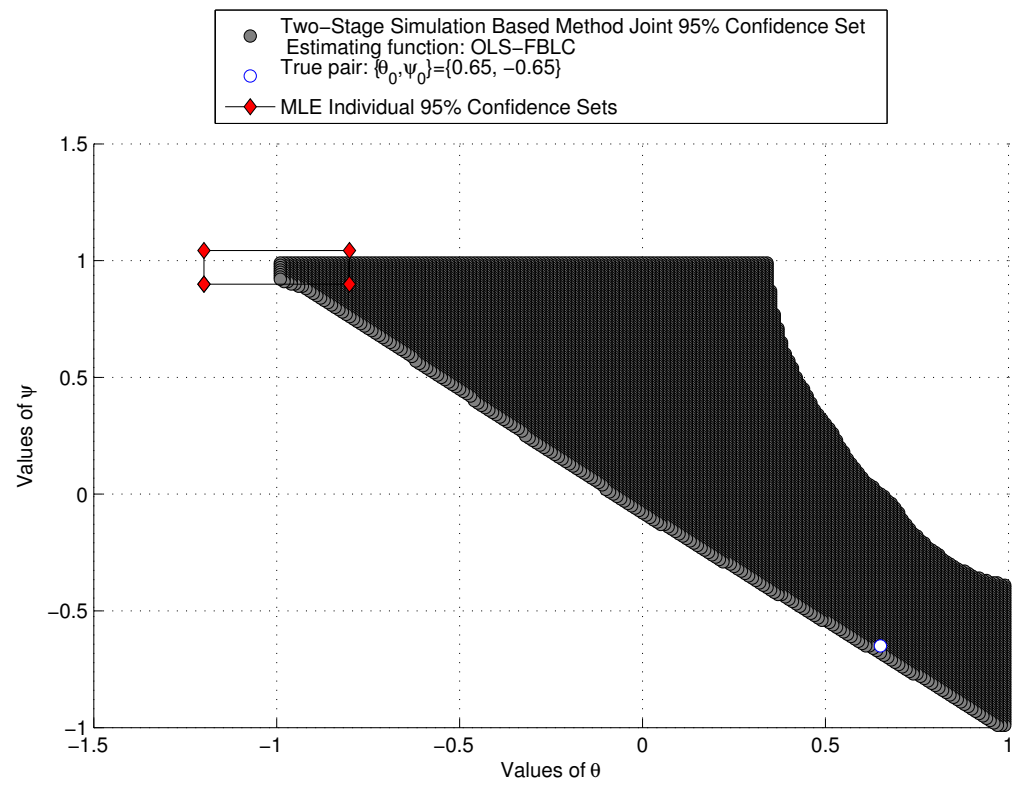


Figure (iii)

Two-Stage Simulation Based Method Joint 95\% Confidence Se Estimating function: OLS-FBLC True pair: $\left\{\theta_{0}, \Psi_{0}\right\}=\{0.65,-0.65\}$

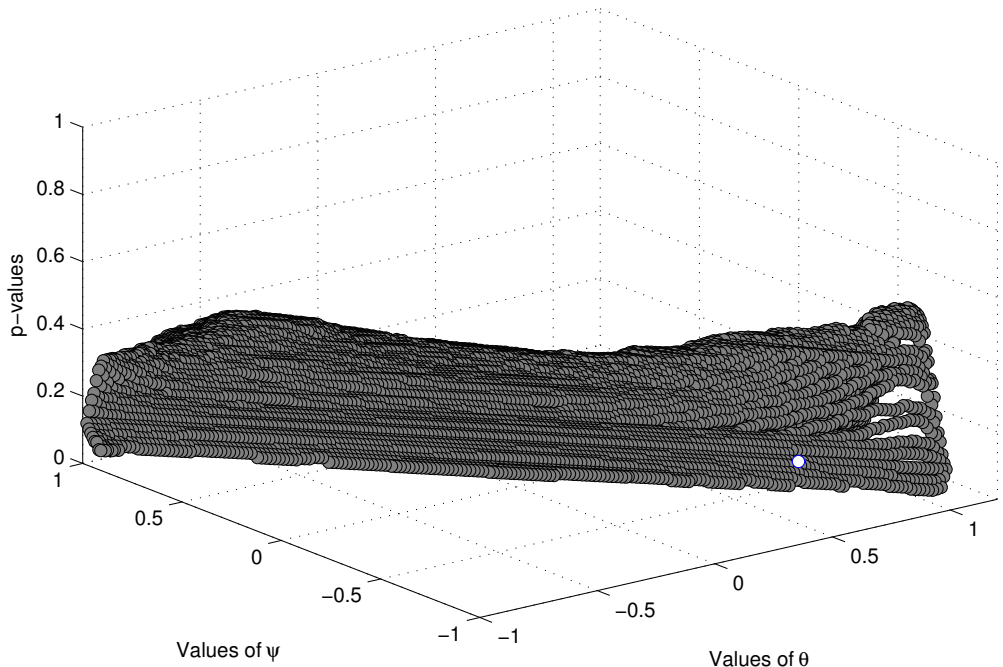

Figure (iv)

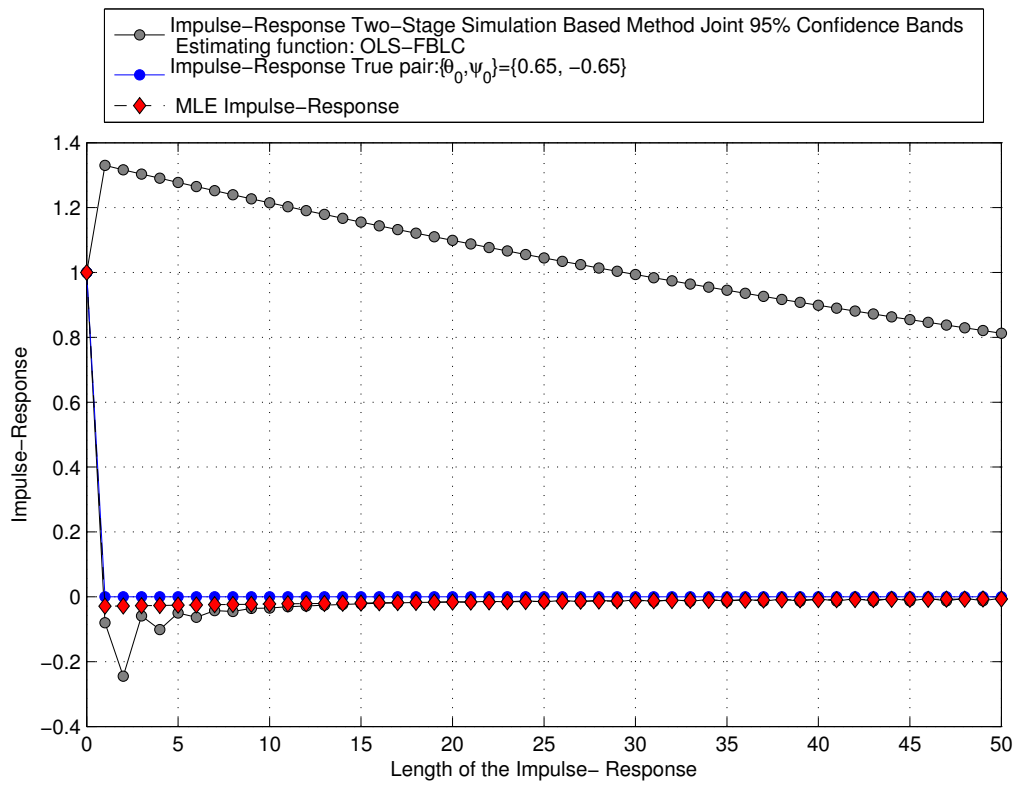




\section{Chapter 2}

\section{Projection- Based}

\section{Impulse-Response Confidence}

\section{Bands for Noth American Crude}

\section{Oil Benchmarks}

\section{$2.1 \quad$ Introduction}

Petroleum, including both crude oil and products made of refined oil, is the most important energy powering source and manufacturing input in the modern economy, despite the increasing use of alternative energy sources. Due to the economic and financial integration among countries, the price of crude oil is an important variable considered in economic forecasting, for instance, by governments and central banks in their macroeconomic projections, by product firms in strategic planning of production-investment decisions and by financial market investors as a hedging commodity for risk management purposes. United States (U.S.) and Canada participate in the oil market as both producers and consumers and crude oil is a major driver of both economies. Refineries in Western Canada run do- 
mestically produced crude oil, while refineries in Quebec and the Atlantic Provinces run primarily imported crude oil. Canada is the largest exporter of crude oil to the U.S. via a system of pipelines, truck tankers and rails.

Predicting the future behaviour of crude oil prices and understanding the impact of oil shocks to the economy have long been relevant research questions. Apart from supply and demand conditions, estimation and forecasting of oil prices are complicated by the effect of non-market related characteristics, such as, regulations, technology and geopolitical considerations. For instance, in early papers, Hamilton (1983 and 1996) investigates the correlation between oil prices and U.S. recessions. Later, Hamilton (2003) and Kilian (2008a, 2008 b and 2008c) focus on methodologies to quantify exogenous oil production shocks and estimate the dynamic effects of these fluctuations on macroeconomic aggregates, such as GDP growth. The main concerns of researches in the literature related to oil prices are whether the impact of oil shocks is temporary or permanent and whether a particular model can offer better performance than a random walk. Hamilton (2009) recognizes the challenge by mentioning that changes in the real price of oil have historically tended to be permanent, difficult to predict and governed by different regimes at different points in time. Despite structural models are very popular in the literature, the effort put on the selection of explanatory variables in this type of models is not always fulfilled by suitable explanatory power and forecasting.

In this chapter, we investigate the persistence of shocks via impulse-response confidence bands for two oil benchmarks priced in U.S. dollars per barrel: the West Texas Intermediate (WTI) for high quality light sweet crude oil delivered at Cushing, Oklahoma, and the Western Canadian Select (WCS) for heavy blended high-TAN (acidic) crude, quoted at the Husky terminal in Hardisty, Alberta. We propose a univariate framework of a two-stage simulation based- method, which considers the series own past history. Our methodology combines Indirect Inference, Monte Carlo (MC) test methods and confidence set projections with focus on Autoregressive Moving Average, ARMA (1,1) processes. 
Our framework offers two main advantages. First, we introduce Stable distributions to be able to capture the heavy tails and asymmetry encountered, since oil shocks are not frequently normally distributed. Stable distributions are a class of distributions first characterized by Paul Lévy (1942), which includes the Normal or Gaussian, Cauchy and Lvy distributions. Non- Gaussian Stable distributions are commonly used to model financial data and they are known to be proxies for Generalized Autoregressive Conditional Heteroscedasticity (GARCH). In addition, Stable distributions admit simulation-based methods, which do not rely on the existence of limiting distributions; see Chambers et al. (1976), and Weron (1996). Second, our framework remains agnostic with respect to the process followed by oil returns by allowing for processes with no persistence, spurious white noise related to root cancelation, heavy persistence, as well as almost unit root. Hence, we are able to capture the range of movement of the series under various observational equivalent processes, meaning pairs of parameters that could generate the same log-likelihood function. This characteristic is related to the work by Sims (2000) and references therein on the indeterminacy of a system of equations resulting in multiple solutions. Also, Lubik and Schorfheide (2003, 2004) provide an extension to a multivariate setting with a likelihood-based estimation of a log-linearized Dynamic Stochastic General Equilibrium (DESG) model without restricting parameters to the determinacy region.

As described in the first chapter, we consider three estimating functions to define auxiliary parameters: (i) two-sided regression with forward and backward looking components (leads and lags),(ii) long-autoregression, and (iii) empirical autocorrelations. Our underlying criterion measures the distance between the parameter estimates obtained from the data and the calibrated values from the first simulation stage. In particular, for non-Gaussian Stable distributions with nuisance parameters, we propose standardizing first and then replacing the nuisance parameters representing skewness and kurtosis by consistent point estimates. Hence, in the second simulation stage, the test statistic is inverted via "Local" Monte Carlo (LMC) test method to obtain a joint confidence set for the parameters of in- 
terest. Refer to Dufour and Khalaf (2002) for details on the LMC. If the confidence region obtained is a closed interval this means that the parameters of interest are well identified. Thus, the resulting confidence set is projected to obtain series of ARMA impulse-responses, from which impulse-response confidence bands are derived.

Most studies available in the literature support studying the real price of oil based on its explanatory power for macroeconomic forecasting of output, for instance, in Baumeister and Kilian (2012) and Alquist, Kilian and Vigfusson (2013). However, Hamilton (2011) argues that nonlinear transformations of the nominal price of oil can reflect threshold responses based on consumer sentiment. In addition, he notes that deflating nominal prices by the Consumer Price Index (CPI) introduces measurement errors that can affect the forecasting results. To have a broad perspective, we examine the returns of nominal series for various samples of weekly and monthly prices, and also study real series in the case of monthly prices. The WIT is analyzed after the U.S. deregulation and post OPEC-administering pricing system from 1986 to 2014. The WCS was launched in 2004 by a group of firms in the Western Canadian Sedimentary Basin oil sands and it is quoted at a discount to the WTI. Due to its availability in Bloomberg, it is studied from 2008 to 2014.

For the purpose of positioning our work in the literature, we have identified four lines of research where oil series has been characterized as a mean reverting process, in particular ARMA: (i) study of long-run persistence in oil prices, (ii) study of oil volatility persistence, (iii) Dynamic Stochastic General Equilibrium (DESG) calibration and (iv) application of structural Vector Autoregression (VAR) family models. The papers identified in the first line of research study oil price dynamics with long series and make modeling decisions considering mean reverting and random walk processes. For instance, Pindyck (1999) studies the long term behaviour of energy prices and concludes that non-structural forecasting frameworks, considering mean reversion and a stochastic fluctuating trend line, perform well for oil prices. Pindyck (1999) does not consider ARMA models per se, but his research supports taking information from long samples. Lee at al. (2006) reject the 
unit root hypothesis for oil prices using Lagrange Multiplier tests, which allow for endogenously determined structural breaks with and without a quadratic trend. Bernard et al. (2010) tests statistical significance and fit of Pindycks (1999) models considering long-run real energy prices and find that a random walk provides the best fit for long-run and real time forecasting of the oil price based on out- of- sample simulation exercises. Recently, Gil-Alana and Gupta (2014) propose a model for crude oil prices incorporating persistence and cyclicity and conclude that cycles repeat every six years approximately.

Oil volatility persistence is studied either by fitting ARMA family models to volatility proxies based on transformations of oil returns, or by applying GARCH family models directly to oil returns. The research by Choi and Hammoudeh (2009), for example, finds evidence of long memory in daily absolute and squared spot and futures returns of crude oil. Also, Charles and Darné (2014) assess the impact of structural changes and outliers on oil volatility persistence applying semi-parametric tests and GARCH family models considering normal, Student-t and skewed Student-t distributions. In the third line of research, DESG models frequently apply calibrations by fitting ARMA family models to oil prices, for instance, in the work of Atkeson and Kehoe (1999) and Leduc and Sill (2004).

The literature applying VAR family models is very extensive and it is mostly dedicated to forecasting in short to middle horizons. For instance, Pyndick (2004) examines the short-run dynamics of commodities by estimating their price volatility and study the effect of shocks to volatility applying VAR models. Killian (2009) proposes endogenizing the price of oil and uses a VAR to decompose fluctuations in the real price of oil. Subsequently, Alquist and Kilian (2010) introduce a general equilibrium model to study the relationship between spot and futures markets of crude oil, and find that the price of crude oil futures is not the most accurate predictor of the spot price in practice. Baumeister and Kilian (2014a) propose the construction of forecast scenarios. Baumeister and Kilian (2012), Alquist, Kilian and Vigfusson (2013), Baumeister and Kilian (2014b) show that including economic determinants of the price of oil, such as changes in oil inventories and production, can 
provide more accurate out-of sample forecasts to the price of oil. Baumeister et al. (2014) and Baumeister and Kilian (2015) apply a forecast combination approach for the real-time forecast of the price of oil. Recently, Baumeister et al. (2015) study univariate Mixed Data Sampling (MIDAS) models and find that there is not typically much lost by ignoring high-frequency financial data in forecasting the monthly real price of oil. Refer to Kilian (2014) for a comprehensive walk through the literature on oil price shocks.

In VAR models, impulse-responses are obtained by either applying simulations, or Bayesian methods. Lütkepohl et al. (2015) provide an overview and evaluation in terms of coverage of the available literature dedicated to impulse-responses for VAR models and propose a new approach based on adjustments to the Bonferroni bands. Our work is related to the research of Staszewska (2007, 2011, 2013), Jordá (2009), Staszewska and Winker (2013), and Jordá and Marcellino (2013) on simultaneous confidence bands and applications to forecasting. It is also related to the working paper by Inoue and Kilian (2014) that proposes constructing asymptotically valid joint confidence sets for structural impulse-responses by inverting a Wald test statistic, regardless of whether the joint distribution of the structural impulse- responses is degenerate or not. In contrast to the VAR literature, our proposed impulse-response confidence bands are compatible with small sample sizes and robust to parameter identification.

The most important conclusion of this chapter is that allowing for asymmetry with Stable distributions helps disentangle the persistence of oil shocks. We find that shocks to oil returns are not permanent, since they are able to dissipate in short to medium time horizons. The resulting impulse-response confidence bands from non-Gaussian Stable distributions for the longest weekly series of oil returns for both WTI and WCS show small immediate responses and short- lived asymmetric feedback effects, which can disappear in two to six weeks, or tend to zero in about twenty weeks. Unfiltered series behave similarly to filtered ones for these two samples. However, with symmetric distributions, such as the Gaussian, we could either overestimate, or underestimate the effect of oil shocks. The 
results obtained are similar when nominal and real monthly samples are considered for both benchmarks. We corroborate that estimations with MLE underestimate the persistence in the series in general.

Time varying parameters may explain estimation uncertainty in ARMA processes as observed. Our impulse-response confidence bands seem well identified despite the presence of parameter uncertainty in oil returns. Similar results in a different context are noted by Magnusson and Mavroeidis (2014). We find that impulse responses are a tool to understand the uncertainty surrounding commonly applied forecasting techniques. In addition, our results can be used to feed more complicated multivariate models.

This chapter is organized as follows. In section 2.2, we introduce our assumptions and methodology. In section 2.3, we analyze the oil prices series of both WTI and WCS. Our results are discussed in section 2.4 and we conclude in section 2.5 .

\section{$2.2 \quad$ Framework}

\subsubsection{Assumptions}

The data can be represented by a zero mean $\operatorname{ARMA}(1,1)$ process of size $T$ with unknown parameters $\theta$ and $\psi$,

$$
X_{t}=\psi X_{t-1}+e_{t}+\theta e_{t-1}, \quad t=1 \ldots T,
$$

with

$$
e_{t}=\sigma u_{t}
$$

where $|\theta|<1$ guarantees invertibility or an Autoregressive $\operatorname{AR}(\infty)$ representation, $|\psi|<1$ ensures stationarity, the joint distribution of $u_{1}, \ldots, u_{t}, \ldots, u_{T}$ is known and $\sigma$ is a nuisance parameter we aim to partial out. For instance, $u_{t}$ can be assumed independent and identi- 
cally distributed with a standardized Stable distribution:

$$
u_{t} \stackrel{i . i . d .}{\sim} \mathcal{S}\left(0,1, \alpha_{s}, \beta_{s}\right), \quad t=1 \ldots T .
$$

We assume that $X_{0}=e_{0}$, where $e_{0}$ is fixed. In addition, Gaussian and Student-t erors are considered.

The characteristic function of a random variable $s$ assumed to follow a Stable distribution $\mathcal{S}\left(\mu, \delta, \alpha_{s}, \beta_{s}\right)$ takes the form:

$$
E[\exp (i t s)]=\left\{\begin{array}{lc}
\exp \left\{-\delta^{\alpha_{s}}|t|^{\alpha_{s}}\left[1-i \beta_{s}\left(\tan \frac{\alpha_{s} \pi}{2}\right)(\operatorname{sign}(t))\right]+i \mu t\right\} & \alpha_{s} \neq 1 \\
\exp \left\{-\delta^{\alpha_{s}}|t|\left[1+i \beta_{s}\left(\frac{2}{\pi}\right)(\operatorname{sign}(t) \ln (|t|))\right]+i \mu t\right\} & \alpha_{s}=1
\end{array}\right.
$$

The Stable distribution above is defined with four parameters: $\mu$ is the location parameter, the density shifts to the right if $\mu$ is positive, or to the left if $\mu$ is negative; $\delta$ is a positive scale parameter, $\alpha_{s}$ is the index of stability or tail index, satisfying $0<\alpha_{s} \leq 2$, and $\beta_{s}$ indicates skewness, satisfying $-1 \leq \beta_{s} \leq 1 .{ }^{1}$ This parametrization admits a discountinuity, since when $\beta_{s}>0$ the mode goes to $+\infty$ as $\alpha_{s} \uparrow 1$, to $-\infty$ as $\alpha_{s} \downarrow 1$ and stays near 0 if $\alpha_{s}=1$

In equation $(3) \operatorname{sign}(t)$ is the sign function satisfying:

$$
\operatorname{sign}(t)= \begin{cases}1 & t>0 \\ 0 & t=0 \\ -1 & t<0 .\end{cases}
$$

Following Nolan (1998), we can show that a standardized Stable distribution can be obtained via a scale and location transformation. This means that the distribution of $u_{t}$

\footnotetext{
${ }^{1}$ This is the most common parametrization available in the literature, introduced by Samorodnitsky and Taqqu (1994).
} 
can be represented as:

$$
\begin{gathered}
u_{t}=\frac{s-\mu}{\delta} \sim^{i . i . d .} \mathcal{S}\left(0,1, \alpha_{s}, \beta_{s}\right), \quad t=1 \ldots T, \\
E[\exp (i t u)]= \begin{cases}\exp \left\{-|t|^{\alpha_{s}}\left[1+i \beta_{s}\left(\tan \frac{\alpha_{s} \pi}{2}\right)(\operatorname{sign}(t))\left(|t|^{1-\alpha}-1\right)\right]\right\} & \alpha_{s} \neq 1, \\
\exp \left\{-|t|\left[1+i \beta_{s}\left(\frac{2}{\pi}\right)(\operatorname{sign}(t) \ln (|t|))\right]\right\} & \alpha_{s}=1 .\end{cases}
\end{gathered}
$$

As Nolan (1999) mentions, critics of the use of Stable distributions point out as limitations that when $\alpha_{s}<2$ and $\beta_{s} \leq 1$, the variance and mean population moments, respectively, do not exist. However, he argues that practitioners commonly use the normal distribution, which has an infinite support. Sample moments will always exists, but they will not converge to the population moments unless $\alpha_{s}=2$ as in the Gaussian distribution.

The importance of the standardized representation, mentioned in Nolan (1998) is that the characteristic function, density and distribution are jointly continuous in the parameters $\alpha_{s}$ and $\beta_{s}$, since $\lim _{\alpha_{s} \rightarrow 1}\left(\tan \frac{\alpha_{s} \pi}{2}\right)\left(|t|^{1-\alpha_{s}}-1\right)=\frac{2}{\pi} \ln |t|$. In addition, $\alpha_{s}$ and $\beta_{s}$ have a clear meaning of the heaviness of the tails and skewness parameters, which describe the characteristics found in the data.

Unknown location and scale parameters can be eliminated by replacing data with standardized observations, considering the sample median and interquartile range:

$$
\hat{X}_{t}=\frac{X_{t}-X[50]}{X[75]-X[25]}, \quad t=1 \ldots T
$$

where $x[Q]$ refers to the $Q$ th quantile of $X_{t}$. With this transformation the distribution of $\hat{X}_{t}$ does not depend on $\mu$ and $\gamma$. The proof of location and scale invariance for the standardized observations can be found in Beaulieu, Dufour and Khalaf (2014).

Our objective is to formulate an exact test for the following joint null hypothesis,

$$
H_{0}\left(\theta_{0}, \psi_{0}\right): \theta=\theta_{0} \quad \text { and } \quad \psi=\psi_{0} .
$$


This test will be inverted to produce a confidence region for the parameters $\theta$ and $\psi$, which will be projected to obtain impulse-response confidence bands.

\subsubsection{Two-Stage Simulation-Based Method}

We apply the two-stage simulation-based method described in the first chapter of this thesis. Our method follows the Indirect Inference methodology, which assumes the existence of an approximate or auxiliary model to describe the data. The q-dimensional parameter

of interest is denoted $\vartheta=\left(\Delta^{\prime}, \sigma\right)$. The auxiliary parameter $\lambda$ of dimension $p \geq q$ can be linked to $\vartheta$ by an exact or limiting binding function, $\lambda=\mathcal{L}(\vartheta)$, which need not have an analytical form. In this particular case, $\Delta^{\prime}=(\theta, \psi)^{\prime}$ contains the $\operatorname{ARMA}(1,1)$ parameters of interest defined above and $\sigma$ is the nuisance parameter we aim to partial out.

We define the estimation of the auxiliary model over the data vector $X$ as

$$
\hat{\lambda}=\hat{\Lambda}(X)
$$

where $\hat{\Lambda}($.$) is the estimating function. The auxiliary parameter \hat{\lambda}$ can be obtained via three estimating functions: (i) OLS estimation with forward-looking and backward-looking components (leads and lags),(ii) OLS estimation of a long-autocorrelation (AR), and (iii) empirical autocorrelations. We refer to these choices as:

i.

$$
\hat{\Lambda}_{f b}(X)=\underset{\gamma}{\operatorname{argmin}} Q(X), \quad \text { with } \quad \gamma=\left(\gamma_{f}^{\prime}, \gamma_{b}^{\prime}\right)^{\prime}
$$

ii.

$$
\hat{\Lambda}_{b}(X)=\underset{\gamma_{0}}{\operatorname{argmin}} Q(X), \quad \text { with } \quad \gamma_{0}=\left(0, \gamma_{b}^{\prime}\right)^{\prime}
$$


where

$$
\begin{array}{r}
Q(X)=\sum_{t=p+1}^{T}\left[X_{t}-\gamma_{f 1} X_{t+1}-\ldots-\gamma_{f p} X_{t+p}\right. \\
\left.-\gamma_{b 1} X_{t-1}-\ldots-\gamma_{b p}^{*} X_{t-p}\right]^{2}
\end{array}
$$

with $\gamma_{f}=\left(\gamma_{f 1}, \ldots, \gamma_{f p}\right)^{\prime}, \gamma_{b}=\left(\gamma_{b 1}, \ldots, \gamma_{b p}\right)^{\prime}$; and

iii.

$$
\hat{\Lambda}_{c}(X)=\left(\hat{\rho}_{1}, \ldots, \hat{\rho}_{p}\right)
$$

where

$$
\hat{\rho}_{j}=\frac{\hat{\operatorname{Cov}}\left(X_{t} X_{t-1}\right)}{\hat{\operatorname{Var}}\left(X_{t}\right)} \quad j=1 \ldots p .
$$

As explained in the first chapter, these estimating functions satisfy the scale invariance property, which allow us to evacuate $\sigma$. We prove that the results obtained are robust to multipying the data by any positive scalar in the univariate case.

The simulation-based counterpart, given values of the parameters of interest $\Delta$, is derived by creating $\mathrm{H}$ simulated paths, each of the same size as the data, applying the same estimation function on each path:

$$
\hat{\lambda}_{h}(\Delta)=\hat{\Lambda}\left(x_{h}(\Delta)\right) \quad h=1, \ldots, H .
$$

The standardized Stable distribution is simulated based on Weron (1996), reproduced here in the following steps. Stable distributions admit simulation-based methods, which do not rely on the existence of limiting distributions; see Chambers et al. (1976), and Weron (1996). Note that the parameters $\alpha_{s}$ and $\beta_{s}$ are calibrated with the estimates of tail index (kurtosis) and skewnes obtained by fitting a Stable distribution to the data.

- Generate a random variable $\mathcal{U}$ uniformly distributed over $\left(\frac{-\pi}{2}, \frac{-\pi}{2}\right)$,

- Generate independenlty an exponential random variable $\mathcal{E}$ with mean 1 , 
- Set $\mathcal{B}_{\alpha_{s} \beta_{s}}=\frac{\arctan \left(\beta_{s} \tan \left(\frac{\pi \alpha_{s}}{2}\right)\right)}{\alpha_{s}}$ and $\mathcal{L}_{\alpha_{s} \beta_{s}}=\left[1+\beta_{s}^{2} \tan ^{2}\left(\frac{\pi \alpha_{s}}{2}\right)\right]^{\frac{1}{2 \alpha_{s}}}$

- Lastly, generate draws from a $\mathcal{S}\left(0,1, \alpha_{s}, \beta_{s}\right)$ dstribution as

$$
\begin{cases}\mathcal{L}_{\alpha_{s} \beta_{s}} \frac{\sin \left(\alpha_{s}\left(\mathcal{U}+\mathcal{B}_{\alpha_{s} \beta_{s}}\right)\right)}{(\cos (\mathcal{U}))^{\frac{1}{\alpha_{s}}}}\left(\frac{\cos \left(\mathcal{U}-\alpha_{s}\left(\mathcal{U}+\mathcal{B}_{\alpha_{s} \beta_{s}}\right)\right)}{\mathcal{E}}\right)^{\frac{1-\alpha_{s}}{\alpha_{s}}} & \alpha_{s} \neq 1, \\ \frac{2}{\pi}\left[\left(\frac{\pi}{2}+\beta_{s} \mathcal{U}\right) \tan \mathcal{U}-\beta_{s} \log \left(\frac{\mathcal{E} \cos (\mathcal{U})}{\frac{\pi}{2}+\beta_{s} \mathcal{U}}\right)\right] & \alpha_{s}=1 .\end{cases}
$$

The estimators obtained are calibrated by computing their average over all the paths:

$$
\Lambda(\Delta)=\frac{1}{H} \sum_{h=1}^{H} \hat{\lambda}_{h}(\Delta) .
$$

A minimum distance objective function is constructed considering the distance betwen the previously estimated $\hat{\lambda}$ and the simulation-calibrated $\Lambda(\Delta)$ auxiliary parameters,

$$
S(\Delta, X)=(\hat{\lambda}-\Lambda(\Delta))^{\prime} \mathcal{I}_{p}(\hat{\lambda}-\Lambda(\Delta))
$$

Note that the p-dimensional identity weighting matrix $\mathcal{I}_{p}$ is chosen to preserve the invariance to scale property. Any alternative choice of weighting matrix should aim to preserve this property.

In the second simulation stage, we apply the "Local" Monte Carlo (LMC) test method to compute the p-values and introduce the test inversion. Asymptotics follow for Stable distributions with calibrated nuisance parameters under the validity of the regularity conditions. Refer to Dufour and Khalaf (2002) for details on the LMC.This is done by adding a new layer of simulation and drawing $L$ replications for each value of $\Delta_{0}$ satisfying the null hypothesis, regardless of the value of $\sigma$. Hence, for each replication $l$ we obtain the auxiliary estimates,

$$
\hat{\lambda}_{l}\left(\Delta_{0}\right)=\hat{\Lambda}\left(x_{l}\left(\Delta_{0}\right)\right)
$$

applying the same estimating function as before, and compute a series of statistics:

$$
S_{l}\left(x_{l}\left(\Delta_{0}\right)\right)=\left(\tilde{\lambda}_{l}\left(\Delta_{0}\right)-\Lambda\left(\Delta_{0}\right)\right)^{\prime} \mathcal{I}_{p}\left(\tilde{\lambda}_{l}\left(\Delta_{0}\right)-\Lambda\left(\Delta_{0}\right)\right)
$$


These statistics are similar to the one in equation (2.2.17), but take the vector of estimated parameters $\hat{\lambda}_{l}\left(\Delta_{0}\right)$ instead of the data-based one. The exchangeability property of the MC test permits using the same vector of calibrated parameters in both statistics.

The empirical $p$-value associated with the null hypothesis is computed as:

$$
\hat{p}\left(\Delta_{0}\right)=\frac{G_{L}+1}{L+1}
$$

where $G_{L}$ denotes the number of times the statistic $S_{l}\left(x_{l}(\Delta)\right)$ is greater than or equal to the data-based statistic $S(\Delta, X)$.

Out of all the pairs tested, we collect those values not rejected by the test, for which the empirically computed $p$-value, $\hat{p}\left(\Delta_{0}\right)$, is higher than the confidence level $\alpha$. With this methodology, we are able to obtain exact p-values for finite samples, and we can control that the probability of type I error is equal to the significance level $\alpha$. As a result, we obtain a joint projection-based confidence set for the $\operatorname{ARMA}(1,1)$ parameters in question satisfying equation (2.2.8). Hence, the resulting joint confidence set, denoted $C S\left(\Delta_{0} ; \alpha\right)$ satisfies the following inequality:

$$
P[\Delta \in C S(\Delta ; \alpha)] \geq 1-\alpha .
$$

The confidence region can take various shapes: (i) a closed interval, (ii) unbounded intervals, (iii) the entire real line, and (iv) an empty set. If we obtain a closed interval, we can conclude that the parameters of interest are well identified. If we have unbounded confidence sets, either unbounded intervals or the entire real line, our method suffers from a partial or complete identification problem. Obtaining an empty confidence set indicates a rejection of the null hypothesis for any pair, and, as a result, a rejection of the empirical counterpart of the binding function selected, and the $\operatorname{ARMA}(1,1)$ specification as a whole.

The empirical impulse-response functions, denoted $g_{i}(\Delta)$ for $i=1, \ldots, m$, are obtained by projecting the joint confidence region for well identified parameters. This implies mini- 
mizing and maximizing $g_{i}(\Delta)$ over the values in $C S\left(\Delta_{0} ; \alpha\right)$ and applying the image function $g_{i}(C S(\Delta ; \alpha))$. Coverage is ensured asymptotically under the same assumptions that provide consistency of the Stable paramter estimates; see Dufour (2006), sections five and six on the asymptotic MC tests.

\subsection{Data}

Daily oil spot prices in U.S. dollars per barrel of the West Texas Intermediate (WTI) for high quality light sweet crude oil delivered at Cushing, Oklahoma are obtained from the webpage of the Energy Information Administration (EIA). The WIT is analyzed after the deregulation and the post OPEC-administering pricing system from May 1986 to Dec. 2014. Alquist, Kilian and Vigfusson (2013) explain that after mid-1980s, due to the U.S. deregulation, the co-movement between the U.S. oil price series, including WTI, refiners acquisition cost for domestically produced oil and for imported crude oil, became stronger. This means that we can take the WIT, since results obtained with other series are expected to be similar due to the high correlation between the various U.S. oil price series.

Daily spot prices in U.S. dollars per barrel of the Western Canadian Select (WCS) for heavy blended high-TAN (acidic) crude, quoted at the Husky terminal in Hardisty, Alberta, are obtained from Bloomberg. The WCS was launched in 2004 by a group of firms in the Western Canadian Sedimentary Basin oil sands and it is quoted at a discount to the WTI. It is available in Bloomberg starting in 2008, and analyzed here from Jan. 2008 to Dec. 2014.

Wednesday prices are taken for the weekly series, and the second week of the month is selected for the monthly series. This is to avoid any calendar effects, such as, the Monday or weekend effect, Friday-effect, and turn-on the- month-effect. Early papers in the financial literature describe these types of effects, for instance, the weekend effect is documented by French (1980), and the monthly-effect, by Ariel (1987). The nominal price series for both 
benchmarks are shown in Figures 1 and 2.

Figure 2.1 shows that WTI prices escalated up to the highest peak during the month of July in 2008, when it reached US\$145 per barrel. Then, the effect of the financial crisis and Great Recession caused a price drop to US\$30.28 in December of the same year. The effect on the WCS observed in Figure 2.2 is similar, considering that at that time it was priced at a lower discount to the WTI than afterwards. Note that the WCS is plotted along with the WTI in Figure 2.2 and the price discount fluctuates during the period of study. ${ }^{2}$

Nominal monthly prices for both benchmarks are deflated using the U.S. Consumer Price Index taking December 2014 as base date. The Consumer Price Index for All Urban Consumers All Items (CPIAUCSL) published by the U.S. Bureau of Labor Statistics is retrieved from the Federal Reserve Bank of St. Louis, Economic Data (FRED).

Our method is implemented taking the returns or log-difference of both real and nominal prices. The following Figures 2.3 and 2.4 show the weekly return of prices for both benchmarks. In Figure 2.3 we can appreciate the large negative return in January 1991 during the Gulf War in the Middle East after the Iraqui invasion to Kuwait in 1990. Also, both figures show the large positive return on July 2008 mentioned above.

Table 2.1 shows a summary of statistics for the various samples selected for both benchmarks. The largest sample size corresponds to the weekly nominal series for the WTI and the smallest corresponds to monthly WCS series. Almost all samples show similar degrees of negative skewness, or distributions with longer left tails, associated with negative returns. However, the WCS weekly nominal series from 2008 to 2014 shows positive skewness or a larger right tail, reflecting the high positive returns before the financial crisis, in particular during July 2008. A sample from 2010 to 2014 is selected for both benchmarks to be able to compare the outcomes of our model in a period post financial crisis and Great Recession.

\footnotetext{
${ }^{2}$ The U.S. subprime mortgage crisis triggered an international financial crisis in 2008. According to the U.S. National Bureau of Economics Research (NBER), the U.S. recession is identified in terms of peaks and troughs from the last quarter of 2007 to the second quarter of 2009. The recession in Canada is reported by Statistics Canada to have lengthened for a shorter period, from the fourth quarter of 2008 to the second quarter of 2009.
} 
Table 2.1: Returns, Summary of Statistics

\begin{tabular}{|c|c|c|c|c|c|c|}
\hline Time Period & WTI Samples & Obs. & Mean & Std. Dev. & Skewness & Kurtosis \\
\hline $1986-2014$ & Weekly Nominal & 1499 & 0.0004841 & 0.0513770 & -0.2191600 & 6.1154000 \\
$1986-2014$ & Monthly Nominal & 347 & 0.0025495 & 0.1013700 & -0.3957300 & 5.3141000 \\
$1986-2014$ & Monthly Real & 347 & 0.0047558 & 0.1026000 & -0.4147000 & 5.3484000 \\
$2010-2014$ & Weekly Nominal & 257 & -0.0017180 & 0.0355510 & -0.1885100 & 3.8245000 \\
\hline Time Period & WCS Samples & Obs. & Mean & Std. Dev. & Skewness & Kurtosis \\
\hline $2008-2014$ & Weekly Nominal & 344 & -0.0027665 & 0.0738300 & 0.3818000 & 6.8182000 \\
$2008-2014$ & Monthly Nominal & 79 & -0.0107990 & 0.1581700 & -0.5188000 & 4.4327000 \\
$2008-2014$ & Monthly Real & 79 & -0.0096169 & 0.1604200 & -0.5600200 & 4.5850000 \\
$2010-2014$ & Weekly Nominal & 257 & -0.0027355 & 0.0625370 & -0.0306090 & 4.0922000 \\
\hline
\end{tabular}

The high level of kurtosis in all samples indicates that historical returns are more clustered around zero, or that the distributions are high peaked. Figures 2.5 and 2.6 diplay the kernel density estimates for the return of weekly nominal prices for the WTI from 1986 to 2014 and the WCS from 2008 to 2014. Both figures show unimodal densities with heavy tails, which provide support to the use of Stable distributions in our method.

\subsection{Results}

Our method is applied to the returns of oil benchmark prices considering both weekly and monthly series. The monthly series are studied in nominal as well as real terms. We consider Stable distributions, including the Gaussian, and the Student-t distribution with 5 and 8 degrees of freedom, which are commonly used in financial time series applications. When assuming a Gaussian and Student-t distributions the series is demeaned first. ${ }^{3}$ For the studies assuming a Stable distribution, the data are replaced by standardized observations as explained in section two. In addition, the parameters $\alpha_{s}$ and $\beta_{s}$ are calibrated with the estimates of tail index (kurtosis) and skewnes obtained by fitting a Stable distribution to the data. These parameters are estimated by applying the program developed by Mark Veillette available freely on the Matlab central website. In terms of the estimation method,

\footnotetext{
${ }^{3}$ Similarly, exogenous variables, if relevant of existing, can be projected out.
} 
the results obtained with Koutrovelis $(1980,1981)$ methodology of estimating an empirical characteristic function and McCulloch (1986) quantile estimation method are very similar, which, according to Nolan (1999) supports the hypothesis that the data are Stable. We compare the outcome of our method considering the three distributions for the weekly prices for both benchmarks. For the other sample sizes, with less than 350 observations, only Gaussian and the Student-t distributions are compared.

The setting of our method is summarized as follows. The total number of auxiliary parameters is set to 8 , which in terms of the three estimating functions means: (i) $\gamma_{f}=\gamma_{b}=4$ in the OLS estimation with forward and backward looking components, or FBLC for shortness, (ii) $\gamma=8$ in the OLS estimation of the long-AR and (iii) $j=8$ in the estimation of empirical autocorrelations. The number of paths in the simulation step is $H=3$, and we conduct a grid search over the four quadrants with a step of 0.01. An identity weighting matrix is used to preserve the invariance to scale of the statistic associated to every choice of the estimating function. Lastly, we select $L=199$ replications in the MMC method to compute the empirical $p$-values, once the test inversion is introduced, and consider a level of significance $\alpha=0.05$. As output of our method we obtain four diagrams: joint $95 \%$ confidence set, confidence surface, the projected impulse-response functions and the corresponding impulse-response confidence bands. Additionally, the diagrams containing our impulse-response confidence bands display the MLE impulse-response function for comparison purposes. In general, estimations with MLE underestimate the persistence in the series studied.

The resulting impulse-response confidence bands seem well identified despite the presence of parameter uncertainty in oil returns. Wide confidence sets for oil returns allow for root cancelations; however, this result does not translate into a widening of our impulseresponse confidence bands. The projected impulse-responses functions obtained with the FBLC estimating function contain sharper oscillatory behaviors than the ones computed with the other two estimating functions. The asymmetries encountered on the behavior of 
the projected impulse-response confidence bands indicate that we are capturing the asymmetry in the effect of shocks. As a robustness check, we run our method on two filtered series by assuming a Stable distribution on the residuals of an $\operatorname{AR}(8)$ fitted to the longest weekly sample for both benchmarks. We find that the results obtained with filtered and unfiltered series are similar. The results for the unfiltered series are presented below and the results for the filtered series are available upon request.

\subsubsection{West Texas Intermediate (WTI)}

Sample: Returns of Weekly Nominal Prices Jan. 1986- Dec. 2014 When the Gaussian distribution is considered, we obtain an empty confidence set with the FBLC estimating function. However, we find closed confidence sets with the long-AR and autocorrelations estimating functions. These confidence sets extend over the second, third and fourth quadrants, including small positive and negative values of $\theta_{0}$, the MA parameter, and small positive and negative values of $\psi_{0}$, the AR parameter. In addition, they do not contain the white noise pair. Figure 2.7 shows the output considering the long-AR estimating function: the $95 \%$ confidence set appears on the first figure, the corresponding confidence surface is on the second one, the impulse-response functions appear on the third one and the confidence bands are presented on the fourth one, jointly with the MLE impulse response function for comparison purposes. We observe that impulse-response confidence bands reach zero on the third week, which means the feedback effect disappears completely afterwards. The study with the t-Student distribution produces empty sets with the three estimating functions for this particular sample.

In contrast, with the non-Gaussian Stable distribution we obtain closed confidence sets with similar shapes for the three estimating functions, which do not contain the white noise pair. These confidence sets appear only on the second quadrant, covering large negative values of $\theta_{0}$ and large positive values of $\psi_{0}$. The confidence set obtained with the long-AR estimating function is the smallest. Figure 2.8 shows the output of our method considering 
again this particular estimating function. For the unfiltered series, we find that the projected impulse-response confidence bands computed with the three estimating functions show small immediate responses and short-lived asymmetric feedback effects, which can last from two to six weeks on the upper band and about fourteen weeks on the lower band. The results obtained with the filtered series are similar, with feedback effects that last up to eight weeks on the upper band and about twenty weeks on the lower band. This means that with the Gaussian distribution we could underestimate the effect of shocks in this particular sample. The MLE impulse-response function also underestimates the persistence on this sample, since it reaches zero on the second week.

\section{Sample: Returns of Monthly Nominal and Real Prices Jan. 1986- Dec.}

2014 The results obtained for the monthly nominal and real series are very close. Only the outcome of our method with the nominal sample is mentioned here and the rest is available upon request. We also obtain one empty confidence set in this case, when the Gaussian distribution is combined with the autocorrelations estimating function. The confidence sets obtained with the long-AR and FBLC estimating functions are narrow and cover the four quadrants; as $\theta_{0}$ increases and gets closer to the unit boundary, $\psi_{0}$ decreases and approaches the unit boundary on the negative side and vice versa. In addition, they do contain the white noise pair. Impulse-response confidence bands obtained with both long-AR and FBLC estimating functions contain oscillatory behaviors and show small, but persistent feedbacks effects, which get close to zero in twenty periods.

When the Student-t distribution is assumed, we obtain closed confidence sets, for the three estimating functions when both 8 and 5 degrees of freedom are selected. The shape of the confidence sets obtained with the long-AR and FBLC estimating functions is similar to the one described above for the Gaussian distribution. However, the confidence set obtained with the autocorrelations estimating function is formed by few independent regions. Figure 2.9 shows the output of our method with autocorrelations as estimating function assuming a Student-t distribution with 8 degrees of freedom, where the confidence set is formed by 
four regions and do not contain the white noise pair. Under both distributional assumptions, impulse-response confidence bands contain oscillatory behaviors and show small, but persistent feedbacks effect which get close to zero in twenty months.

Sample: Returns of Weekly Nominal Prices Jan. 2010- Dec. 2014 Lastly, for this sample we obtain closed confidence sets assuming both Gaussian and Student-t distribution with 5 and 8 degrees of freedom, using the three estimating functions. Among the three confidence sets obtained with the Gaussian distribution, the long-AR estimating function gives the narrower. The confidence sets obtained with the Student-t distribution with both degrees of freedom are similar to those obtained with the Gaussian distribution. They contain the white noise pair and touch the four quadrants. The exception is the confidence set obtained with the Student-t distribution with 8 degrees of freedom selecting the long-AR estimating function because it is the only one formed by two independent closed regions. The output of our method in this particular case is displayed in Figure 2.10. Also, impulse-response confidence bands show small and persistent feedback effects which get close to zero in about twenty weeks. Despite all impulse-response confidence bands contain oscillatory behaviors in some extent, the ones computed with the FBLC estimating functions display the sharpest oscillatory behavior.

\subsubsection{Western Canadian Select (WCS)}

We obtain closed intervals in all the samples studied for this benchmarks, which cover the four quadrants. The results obtained with the Student-t distribution are in line with the ones obtained with the Gaussian distribution. These results are available upon request, but not discussed here for shortness.

Sample: Returns of Weekly Nominal Prices May 2008- Dec.2014 In the studies conducted considering the assumption of normality, the confidence sets obtained with the three estimating functions have similar shapes; as $\theta_{0}$ increases and gets closer to 
the unit boundary, $\psi_{0}$ decreases and approaches the unit boundary on the negative side and vice versa. In addition, the three confidence sets allow for the white noise process. Impulseresponse confidence bands show that both the immediate response and the feedback effects are small, but do not disappear before the twentieth month. Figure 2.11 presents the output of our method considering the autocorrelations estimating function.

The confidence sets obtained considering the Stable distribution are narrower than the ones obtained with the Gaussian distribution, especially in the fourth quadrant. In particular, the confidence sets computed with the FBLC and the long-AR estimating functions also allow for the white noise process. In contrast, the confidence set obtained with the autocorrelations is smaller than the one described before and covers only the second quadrant. This means that we have negative values of the MA parameter $\theta_{0}$, positive values of the AR parameter $\psi_{0}$, and the white noise process is not contained. The output for this particular estimating function is displayed in Figure 2.12. Impulse-response confidence bands obtained with the autocorrelations are smoother than the other ones. In general, the impulse-response confidence bands are narrower and closer to zero than those obtained with the Gaussian distribution and the feedback effect on the unfiltered series last from two to four weeks on the upper band and approximately twenty weeks on the lower band. The results obtained with the filtered series are similar, with feedback effects that last from three weeks on the upper band to about twenty weeks on the lower band. As a result, with the Gaussian distribution we could overestimate the effect of shocks in this particular sample. The MLE impulse-response function underestimates the persistence in this sample, since it reaches zero on the second week.

\section{Sample: Returns of Monthly Nominal and Real Prices May 2008- Dec.}

2014 The results obtained with the monthly nominal and real samples assuming a Gaussian distribution are very similar. Only the outcome of our method with the nominal sample is mentioned here and the rest is available upon request. We obtain much wider confidence sets than those described above, especially with the autocorrelations estimating function 
on the first and fourth quadrants, which is observed in Figure 2.13. These results translate into wider impulse-response confidence bands with a larger and sharper oscillatory effects on both bands that have a parallel behaviour for the long-AR and Autocorrelation estimating functions, but asymmetric behaviour for the FBLC estimating function. We find that the persistence of this series tends to zero in about twenty months.

Sample: Returns of Weekly Nominal Prices Jan. 2010- Dec. 2014 Lastly, for this weekly sample assuming a Gaussian distribution, the confidence sets obtained with the three estimating function show a similar shape to the ones described for the weekly sample starting in 2008. Impulse-response confidence bands show a large immediate response and some oscillatory feedback effects with a close parallel behavior in both bands, which tend zero in nearly twenty periods. This can be observed in Figure 2.14, which considers the FBLC estimating function.

\subsection{Conclusion}

The objective of this chapter is to investigate the persistence of shocks via impulseresponse confidence bands for two oil benchmarks priced in U.S. dollars per barrel. We examine the returns of nominal series for various samples of weekly and monthly prices, considering data from 1986 to 2014 for the WTI and from 2008 to 2014 for the WCS. In addition, real series are examined for both benchmarks by deflating monthly prices. Our proposed univariate method delivers simultaneous impulse-response confidence bands for ARMA $(1,1)$ process by combining Indirect Inference, MC test methods and confidence sets projections. The two main advantages of our two-step simulation-based method are: allowing for heavy tails and asymmetry with Stable distributions, and remaining agnostic with respect to the process followed by oil returns. We conclude that allowing for asymmetry helps disentangle the persistence of oil shocks. We find that shocks to oil returns are not permanent, but dissipate in short to medium time frames. The resulting impulse- 
response confidence bands from non-Gaussian Stable distributions for the longest weekly series of oil returns for both WTI and WCS show small immediate responses and short-lived asymmetric feedback effects, which can disappear in two to six weeks, or tend to zero in approximately twenty weeks. We find that unfiltered series behave similarly to filtered ones for these two samples. In contrast, with symmetric distributions we could underestimate, or overestimate the effect of shocks for the WTI and the WCS, respectively. Our results are similar when nominal and real monthly samples are considered for both benchmarks. We find that shorter monthly and weekly samples show higher persistence, which can last about twenty periods for both benchmarks. Student-t distributions produce non-empty confidence sets and impulse responses with oscillatory and persistence behaviours with monthly series as well as the shorter weekly series for both benchmarks. It is noteworthy that the resulting impulse-response confidence bands seem well identified despite the presence of parameter uncertainty in oil returns. In addition, wide confidence sets for oil returns allow for root cancelations; however, this result does not translate into a widening of our impulse-response confidence bands. Our results indicate that impulse-response functions provide the advantage of being better identified to generate forecast. We corroborate that MLE underestimates the persistence in the series studied. Lastly, our findings support the usefulness of impulse-responses obtained from asymmetric and heavy tailed distributions, which leaves the door open for further research. 


\section{References}

[1] Energy Information Administration (EIA). WTI prices in U.S. dollars per barrel Jan.1986-Dec. 2014. Retrieved from eia.gov.

[2] Alquist, R. and L. Kilian (2010). What do We Learn From the Price of Crude Oil Futures? Journal of Applied Econometrics 25, 539-573.

[3] Alquist, R., L. Kilian and R.J. Vigfusson (2013). Forecasting the Price of Oil, in Handbook of Economic Forecasting, Vol. 2 Part A, 427-507, edited by Graham Elliott and Allan Timmermann, Elsevier B.V.

[4] Ariel, Robert A. (1987). A Monthly Effect in Stock Returns. Journal of Financial Economics 18, 161-147.

[5] Beaulieu M-C., Dufour J.-M. and L. Khalaf (2014). Exact Confidence Set Estimation and Goodness of Fit Test Methods for Asymmetric Heavy Tailed Stable Distributions. Journal of Econometrics 181, 3-14.

[6] Baumeister, C. and L. Kilian (2012). Real-Time Forecasts of the Real Price of Oil. Journal of Business \& Economic Statistics 30, 326-336

[7] Baumeister, C. and L. Kilian (2014a). Real-Time Analysis of Oil Price Risks Using Forecast Scenarios. IMF Economic Review, 62,120-145, International Monetary Fund.

[8] Baumeister, C. and L. Kilian (2014b). What Central Bankers Need to Know About Forecasting Oil Prices. International Economic Review 55, 869889. 
[9] Baumeister, C., L. Kilian and T.K. Lee (2014). Are There Gains from Pooling RealTime Oil Price Forecasts? Energy Economics 46, 533-543.

[10] Baumeister, C. and L. Kilian (2015). Forecasting the Real Price of Oil in a Changing World: A Forecast Combination Approach. Journal of Business and Economics Statistics, 33, 338-351.

[11] Baumeister, C. P. Gurin and L. Kilian (2015). Do High-Frequency Financial Data Help Forecast Oil Prices? The MIDAS Touch at Work. International Journal of Forecasting $31,238-252$.

[12] Bernard, J.-T., J.-M. Dufour, L. Khalaf and M. Kichian (2012). An IdentificationRobust Test for Time-Varying Parameters in the Dynamics of Energy Prices. Journal of Applied Econometrics 27, 603-624.

[13] Bloomberg L.P. (2015). Western Canadian Select (WCS) prices in U.S. dollars per barrel May.1986-Dec. 2014. Bloomberg Electronic Database. Department of Economics, Carleton University, 1125 Colonel By Drive, Ottawa ON K1S 5B6, Canada, Feb./2/2015.

[14] Chambers, J. M., C. L.Mallows and B. W. Stuck (1976). A Method for Simulating Stable Random Variables. Journal of the American Statistical Association 71, 340344.

[15] Charles, Amelié and Olivier Darné (2014). Volatility Persistence in Crude Oil Markets. Energy Policy 65, 729-742.

[16] Choi, K. and S. Hammoudeh (2009). Long Memory in Oil and Refined Product Markets. The Energy Journal 30,97-116.

[17] Dufour J.-M. and Lynda Khalaf (2002). Simulation Based Finite and Large Sample Tests in Multivariate Regressions. Journal of Econometrics 111, 303-322. 
[18] Dufour J.-M. (2006). Monte Carlo Tests with Nuisance Parameters: A General Approach to Finite-Sample Inference and Nonstandard Asymptotics in Econometrics. Journal of Econometrics 133, 443-478.

[19] Farmer, Roger E.A. and Jang-Ting Guo (1994). Real Business Cycles and the Animal Spirits Hypothesis. Journal of Economic Theory 63, 42-72.

[20] French, Kenneth R. (1980). Stocks Returns and the Weekend Effect. Journal of Financial Economics 8,55-69.

[21] Gil-Alana, Luis A. and Rangan Gupta (2014). Persistence and Cycles in Historical Oil Price Data. Energy Economics 45, 511-516.

[22] Hamilton, J.D. (1983). Oil and the Macroeconomy since World War II. Journal of Political Economy 91, 228248.

[23] Hamilton, J.D. ( 1996). This is What Happened to the Oil Price-Macroeconomy Relationship. Journal of Monetary Economics 38, 215220.

[24] Hamilton, J.D.(2003). What Is an Oil Shock? Journal of Econometrics 113, 363-398.

[25] Hamilton, J.D. (2009). Understanding Crude Oil Prices. The Energy Journal 30, 179205. International Association of Energy Economics (IAEE).

[26] Hamilton J.D. (2011). Nonlinearities and the Macroeconomic Effects of Oil Prices. Macroeconomic Dynamics 15, 364-378.

[27] Inoue, A. and Kilian, L. (2013). Inference on Impulse Response Functions in Structural VAR Models. Journal of Econometrics, 177, 113.

[28] Inoue, A. and Kilian, L. (2014). Joint Confidence Sets for Structural Impulse Responses. Working paper. 
[29] International Energy Agency (IEA) (2014). Key World Energy Statistics. Retrieved from http://www.iea.org/publications/freepublications/publication/Key

World2014.pdf

[30] Kilian, Lutz (2008a). A Comparison of the Effects of Exogenous Oil Supply Shocks on Output and Inflation in the G7 Countries. Journal of the European Economic Association, 6, 78-121.

[31] Kilian, Lutz ( 2008b). The Economic Effects of Energy Price Shocks. Journal of Economic Literature, 46, 871-909.

[32] Kilian, Lutz (2008c). Exogenous Oil Supply Shocks: How Big Are They and How Much Do They Matter for the U.S. Economy? Review of Economics and Statistics, 90, $216-40$

[33] Kilian, Lutz (2009). Not All Oil Price Shocks Are Alike: Disentangling Demand and Supply Shocks in the Crude Oil Market. American Economic Review 99, 1053-1069.

[34] Kilian, Lutz (2014). Oil Price Shocks: Causes and Consequences. Annual Review of Resource Economics 6, 133-154.

[35] Leduc S. and K. Sill (2004). A Quantitative Analysis of Oil Price Shocks, Systematic Monetary Policy, and Economic Downturns. Journal of Monetary Economics 51, 781808

[36] Lee, J., J.A.List and M.C. Strazicich (2006). Non-Renewable Resource Prices: Deterministic or Stochastic Trends? Journal of Environmental Economic Management 3, 354370.

[37] Lévy, P. (1924) .Théorie de L'Addition des Variables Aléatoires. Bulleting de la Société de France 52, 49-85. 
[38] Lubik, T.A and F. Schorfheide (2003).Computing Sunspot Equilibria in Linear Rational Expectations Models. Journal of Economic Dynamics 83 Control 28, 273-285.

[39] Lubik, T.A and F. Schorfheide (2004). Testing for Indeterminancy: An Application to U.S. Monetary Policy. The American Economic Review 94, 190-217.

[40] Lü tkepohl, H., Anna Staszewska-Bystrova and Peter Winkerc (2015). Comparison of Methods for Constructing Joint Confidence Bands for Impulse Response Functions. International Journal of Forecasting 31, 782-798.

[41] Magnusson, L. M. and S. Mavroeidis (2014). Identification Using Stability Restrictions. Econometrica 82, 1799-1851.

[42] Nolan, J.P. (1998). Parametrizations and Modes of Stable Distributions. Statistics and Probability Letters 38, 187-195.

[43] Nolan, J.P. (1999). Fitting Data and Assessing Goodness-of-fit with Stable Distributions, retrieved from http://academic2.american.edu/ jpnolan/stable/stable.html.

[44] Nolan, J. P. (2003). Stable Distributions: Models for Heavy Tailed Data. Birkhuser, Boston, MA.

[45] Nolan, J. P. (2005). Modeling Financial Fata with Stable Distributions, retrieved from http://academic2.american.edu/ jpnolan/stable/stable.html

[46] Pindyck, Robert S. (1999). The Long Run Eolution of Energy Prices. The Energy Journal 20, 1-27.

[47] Statistics Canada (2012). Canada Year Book, Energy Chapter, retrieved from http://www.statcan.gc.ca/pub/11-402-x/2012000/pdf/energy-energie-eng.pdf.

[48] Staszewska, A. (2007). Representing Uncertainty About Impulse Response Paths: The Use of Heuristic Optimization Methods. Computational Statistics and Data Analysis, 52,121132 . 
[49] Staszewska-Bystrova, A. (2011). Bootstrap Prediction Bands for Forecast Paths from Vector Autoregressive Models. Journal of Forecasting, 30, 721735.

[50] Staszewska-Bystrova, A. (2013). Modified Scheffs Prediction Bands. Journal of Economics and Statistics, 233, 680-690.

[51] Staszewska-Bystrova, A. and P. Winker (2013). Constructing Narrowest Pathwise Bootstrap Prediction Bands Using Threshold Accepting. International Journal of Forecasting, 29, 221-233.

[52] Sims, Christopher A. (2001). Solving Linear Rational Expectations Models. Computational Economics 20, 1-20.

[53] US. Bureau of Labor Statistics, Consumer Price Index for All Urban Consumers: All Items [CPIAUCSL], Index 1982-84=100. Retrieved from FRED, Federal Reserve Bank of St. Louis.

https://research.stlouisfed.org/fred2/series/CPIAUCSL/, April/13/ 2015.

[54] Veillette, Mark. Alpha stable distributions for Matlab, package downloaded from Matlab Central website http://www.mathworks.com/matlabcentral/fileexchange/ 37514-stbl-alpha-stable-distributions-for-matlab.

[55] Weron, R. (1996). On the Chambers-Mallows-Stuck Method for Simulating Skewed Stable Random Variables. Statistics and Probability Letters 28, 165-171. 
Figure 2.1: West Texas Intermediate

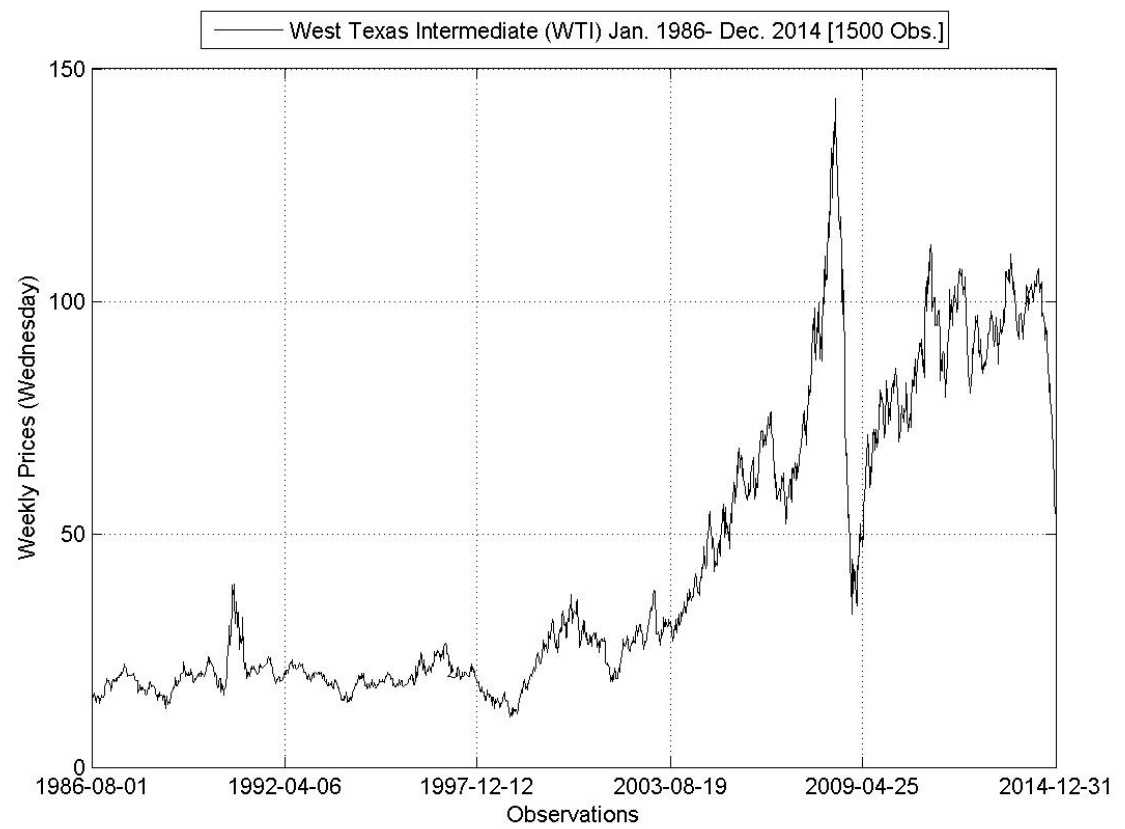

Figure 2.2: Western Canadian Select

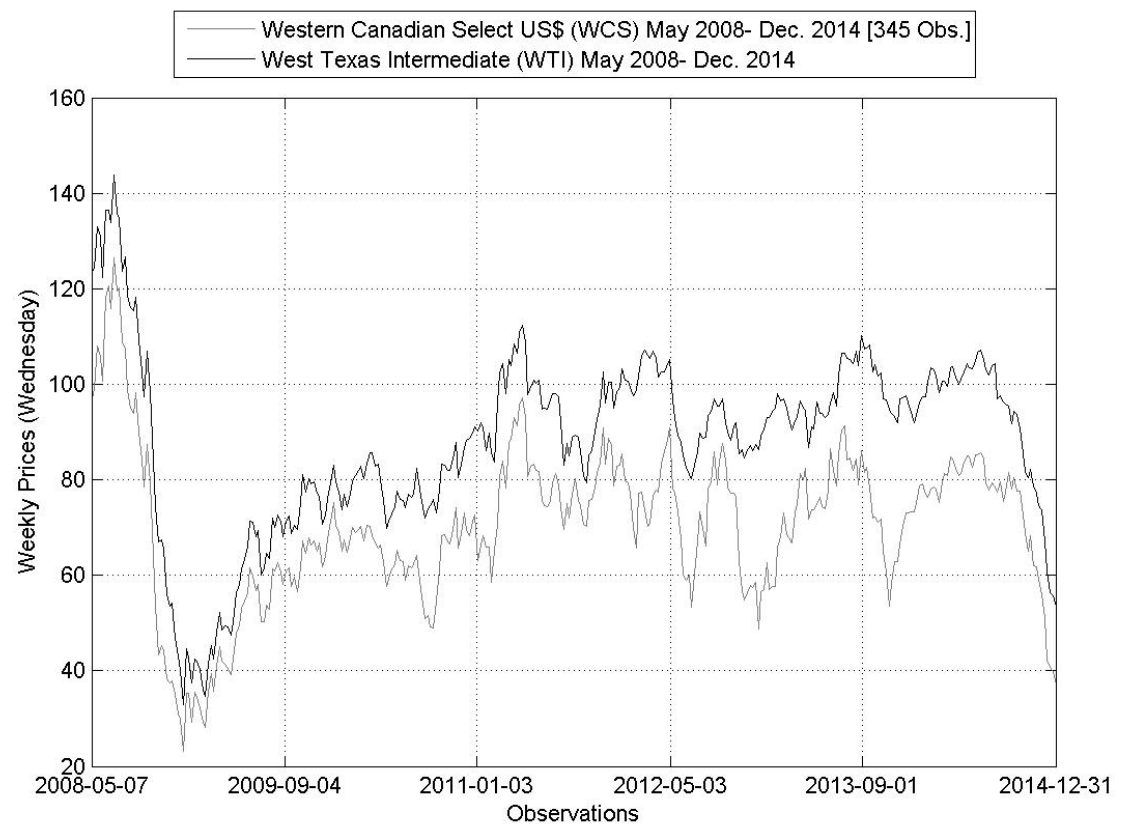


Figure 2.3: West Texas Intermediate

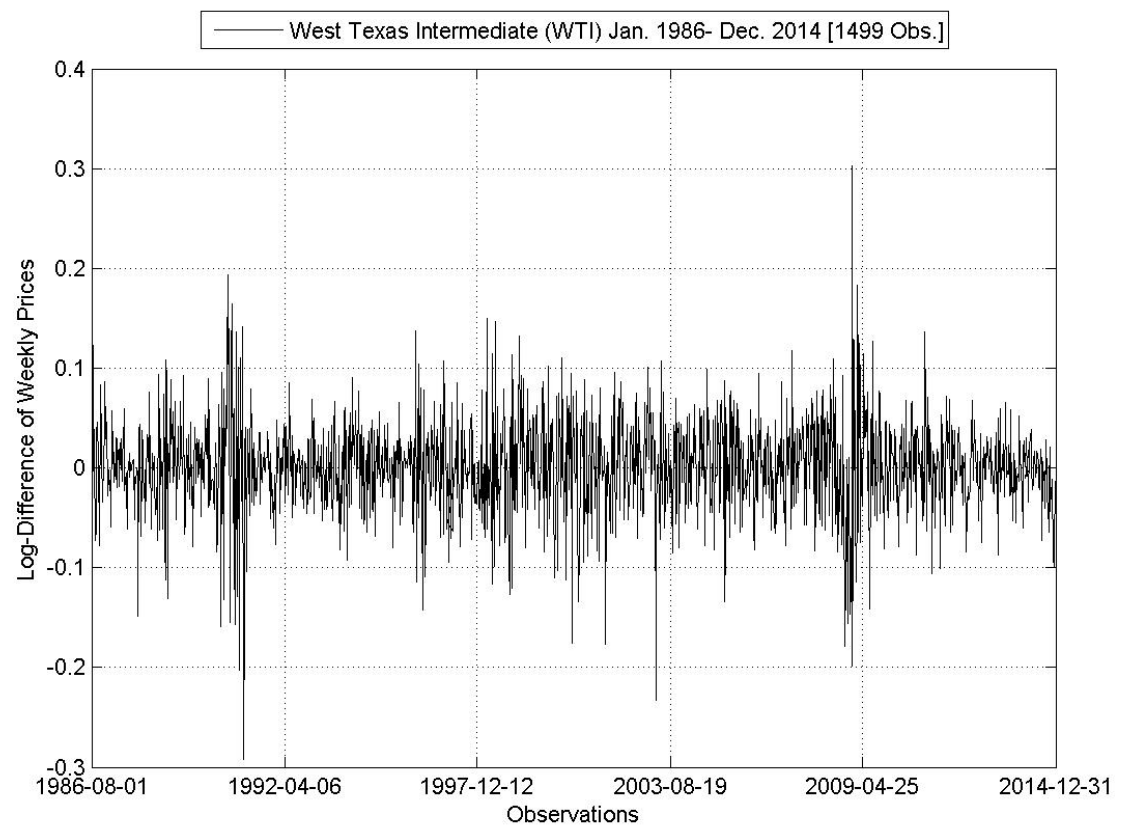

Figure 2.4: Western Canadian Select

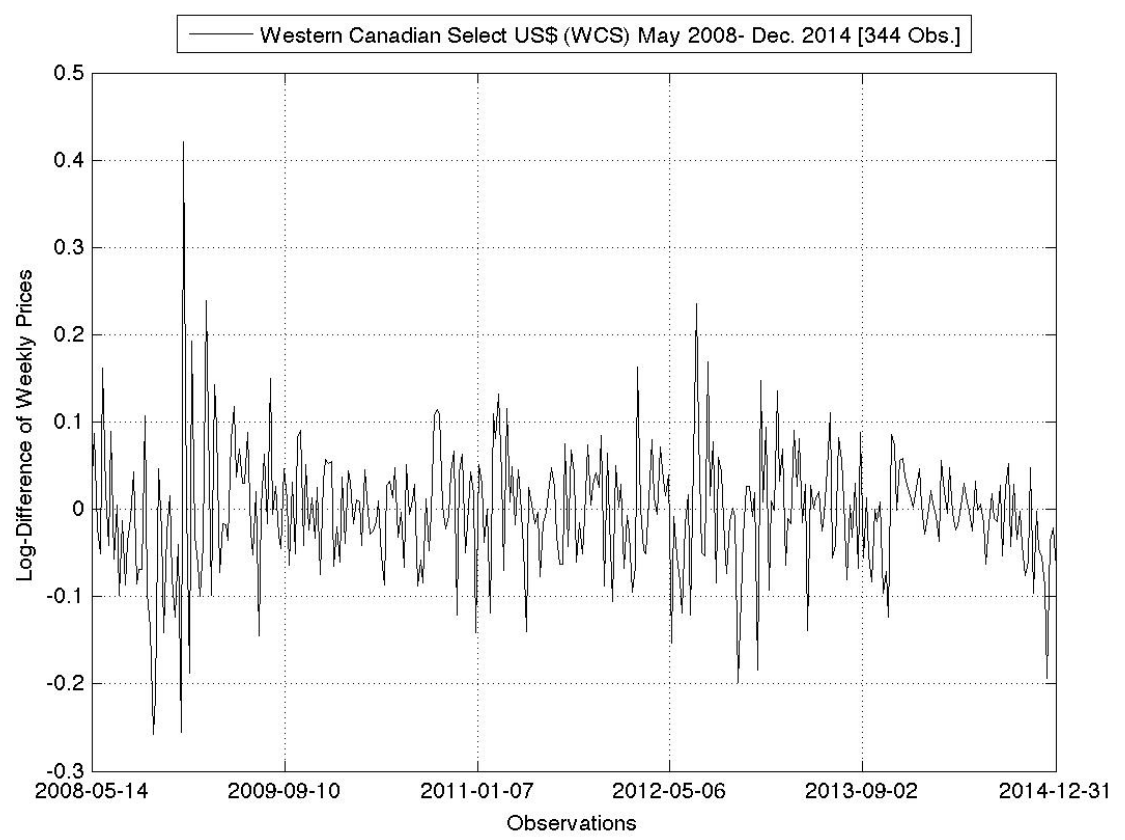


Figure 2.5: West Texas Intermediate: Kernel Density Estimate

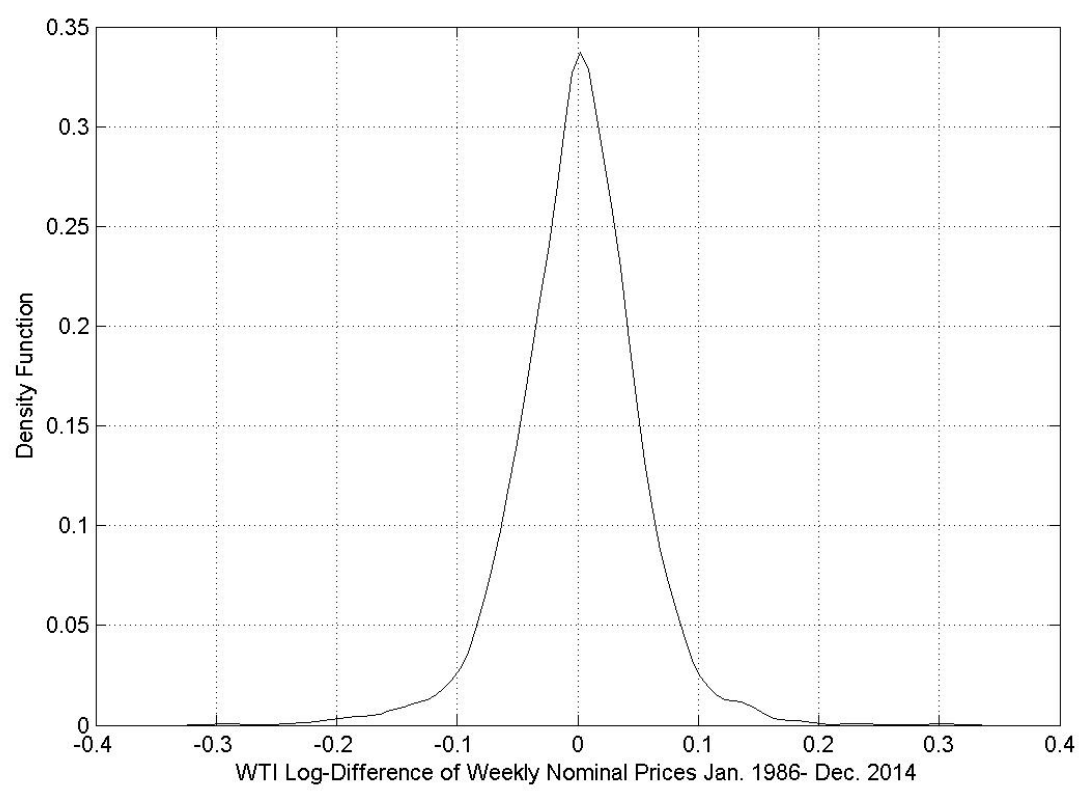

Figure 2.6: Western Canadian Select: Kernel Density Estimate

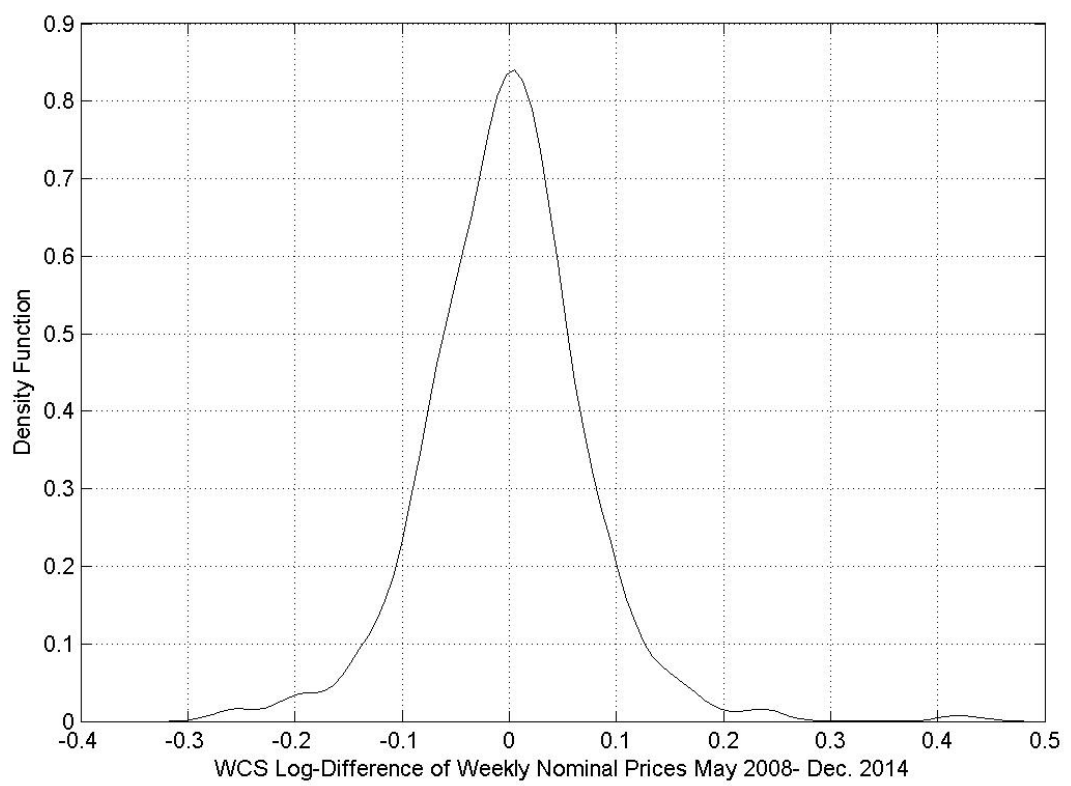


Figure 2.7: West Texas Intermediate (WTI) Jan. 1986- Dec. 2014 (Weekly). Gaussian Distribution

Figure (i)

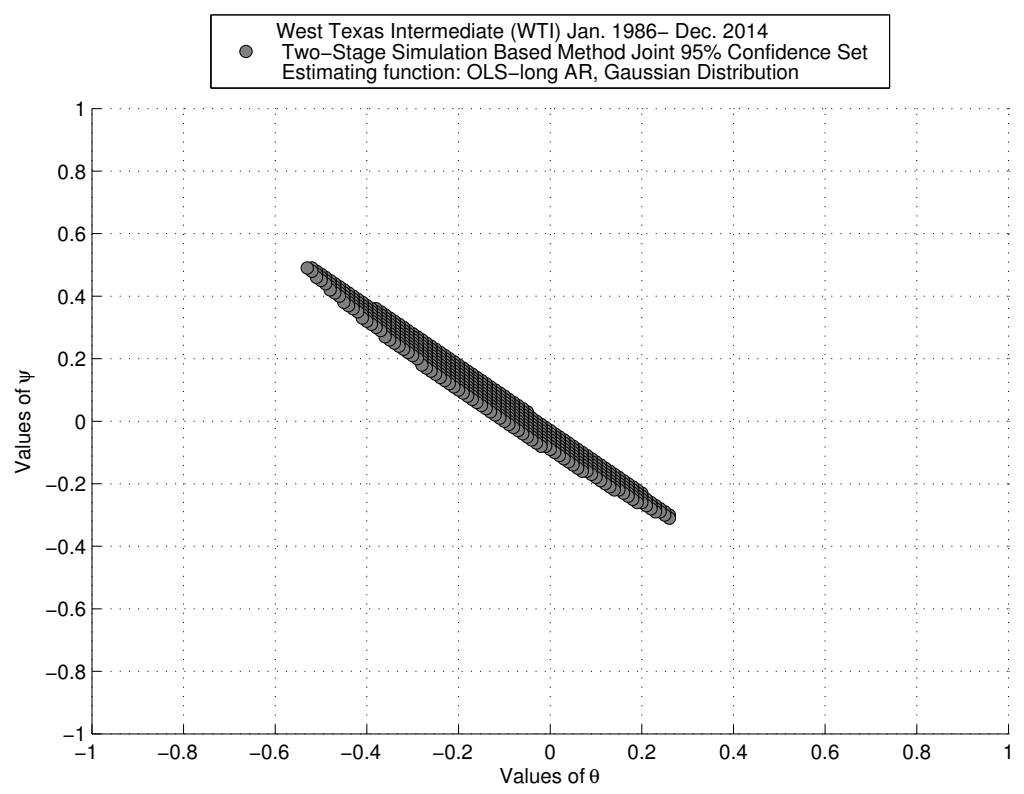

Figure (ii)

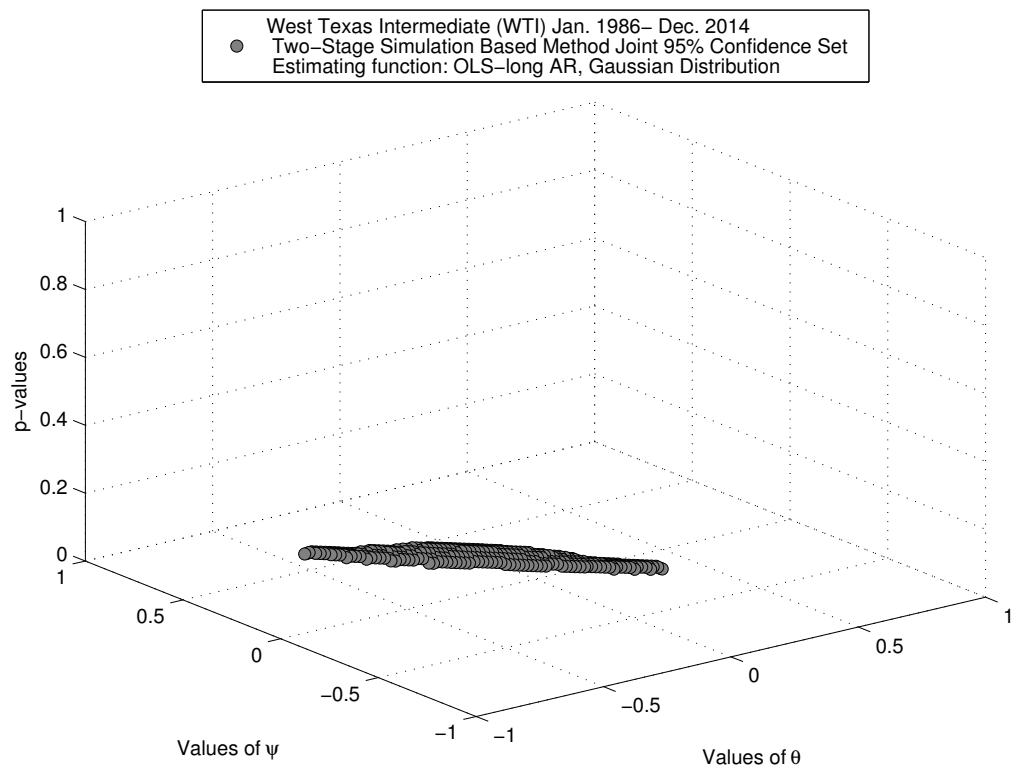


Figure (iii)

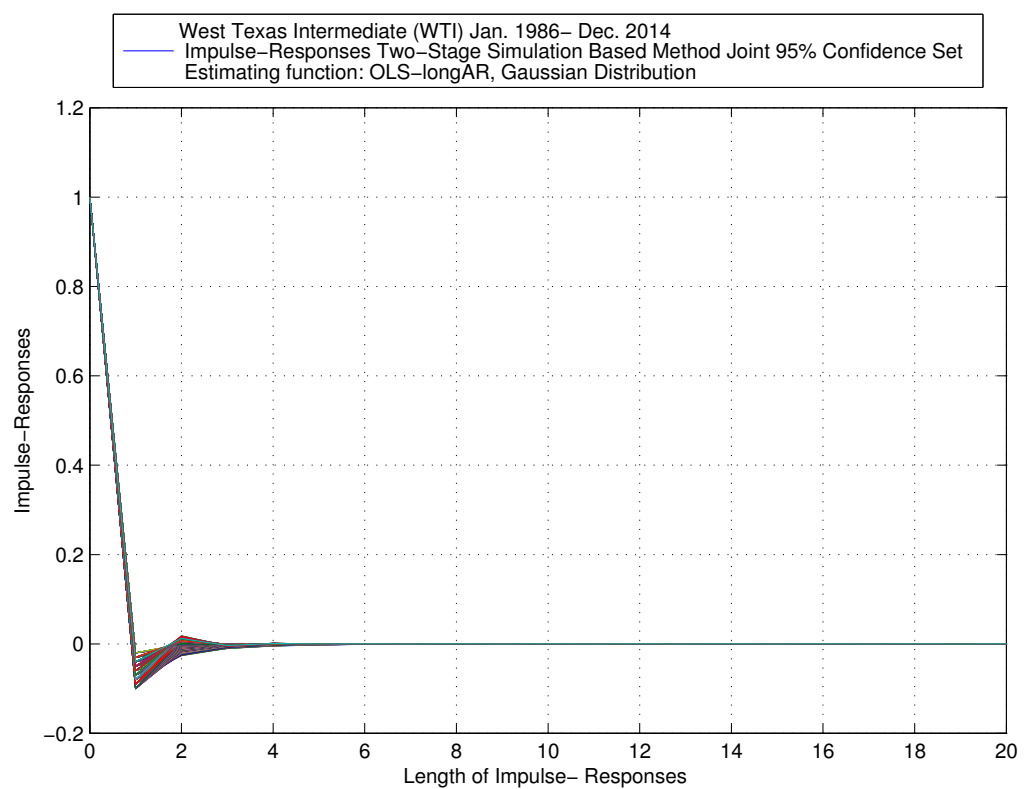

Figure (iv)

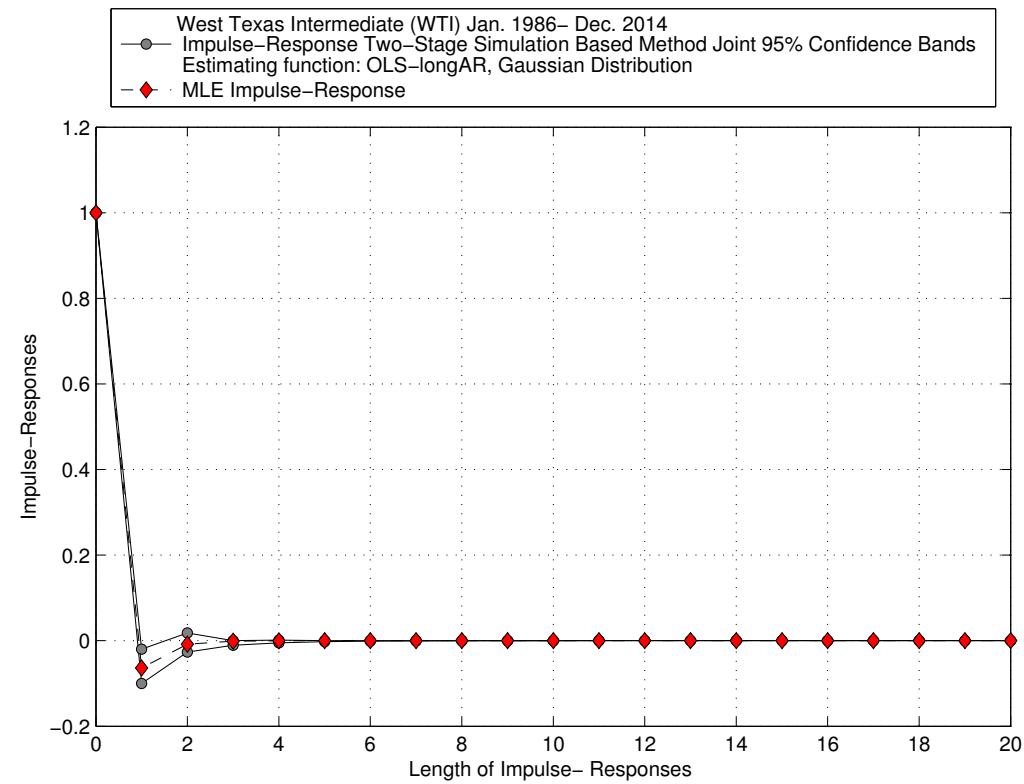


Figure 2.8: West Texas Intermediate (WTI) Jan. 1986- Dec. 2014 (Weekly). Stable Non-Gaussian distribution

Figure (i)

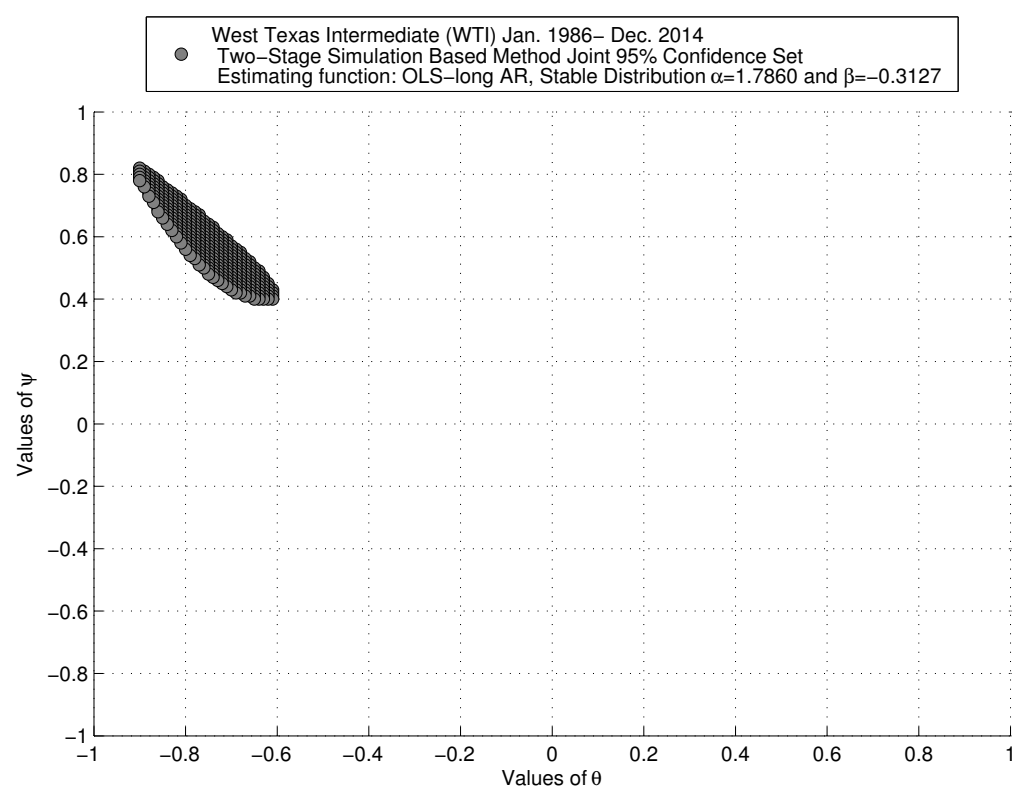

Figure (ii)

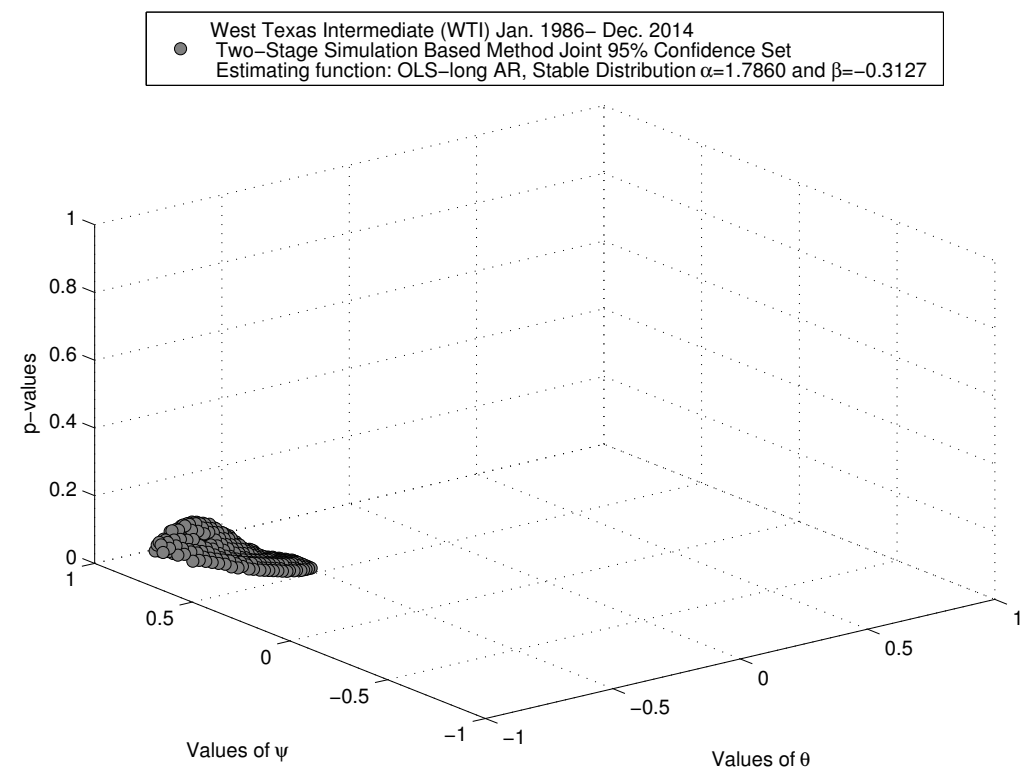


Figure (iii)

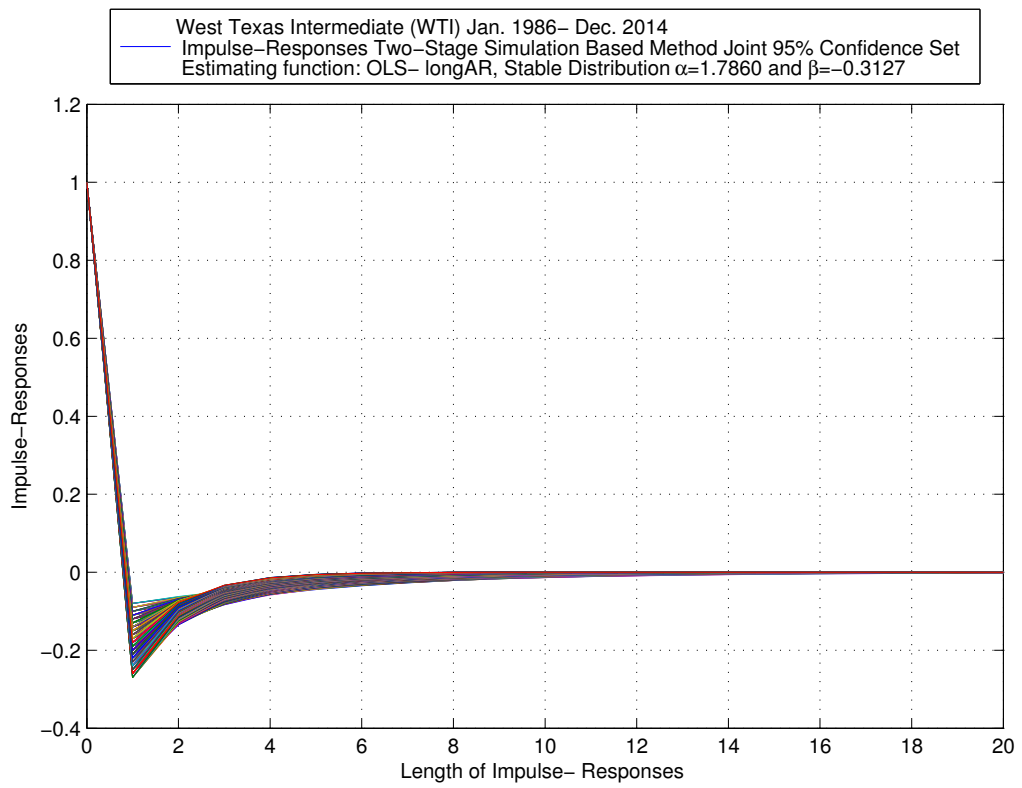

Figure (iv)

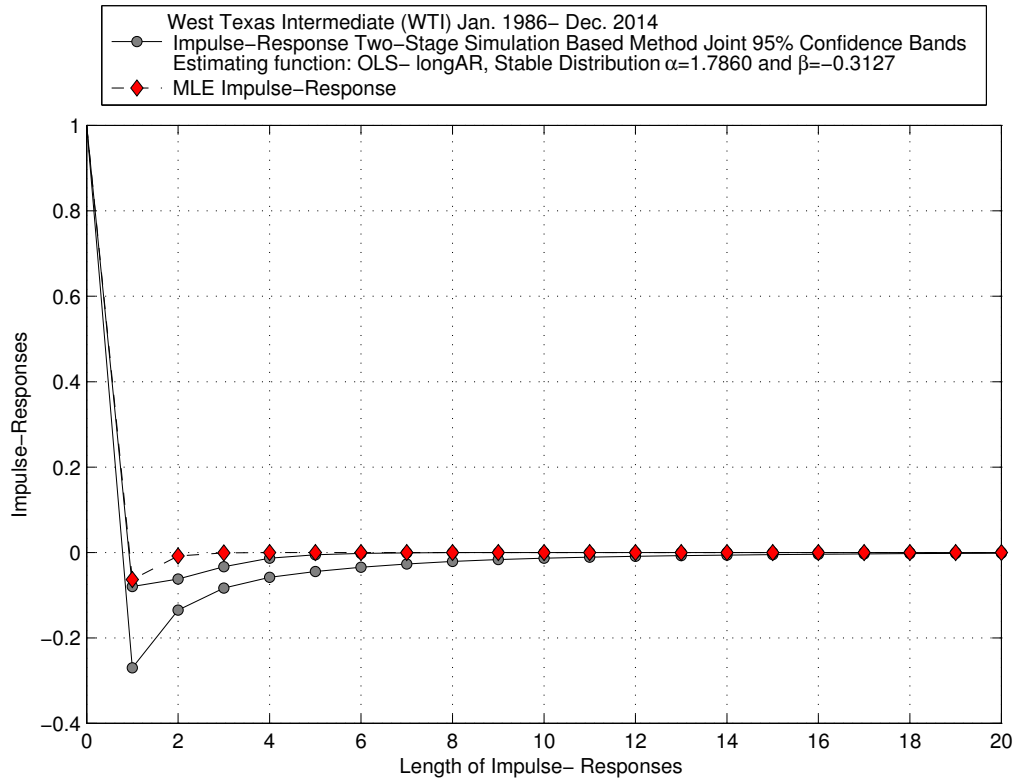


Figure 2.9: West Texas Intermediate (WTI) Jan. 1986- Dec. 2014 (Monthly). Student-t Distribution with 8 Degrees of Freedom

Figure (i)

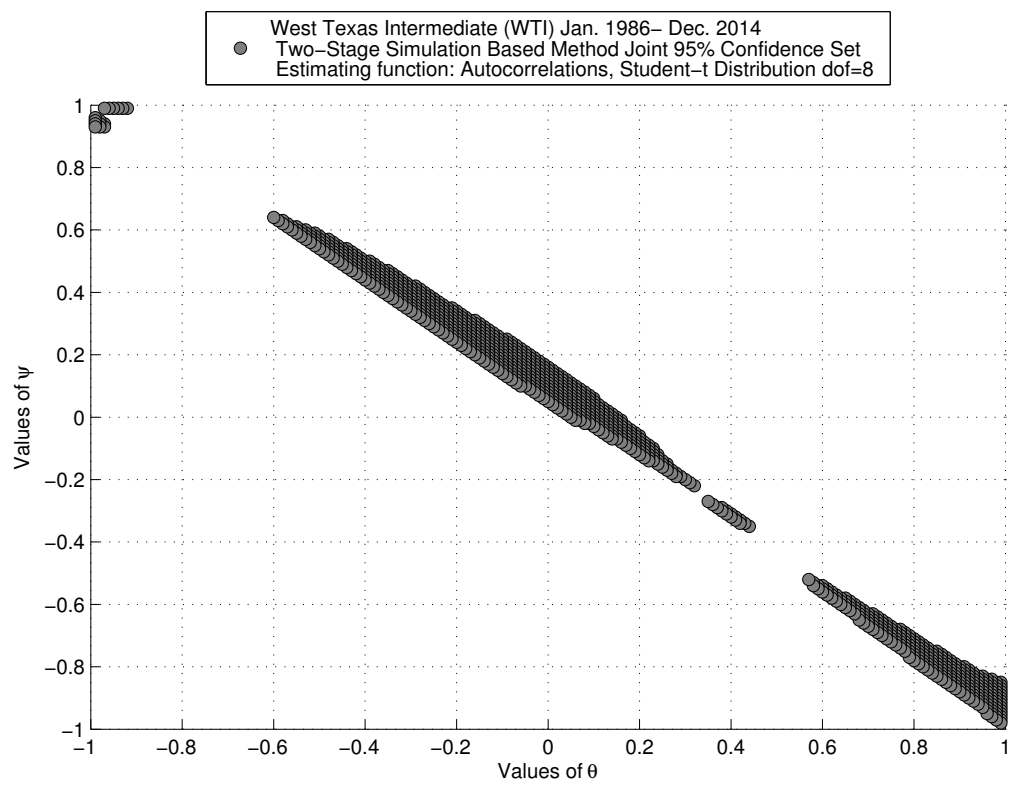

Figure (ii)

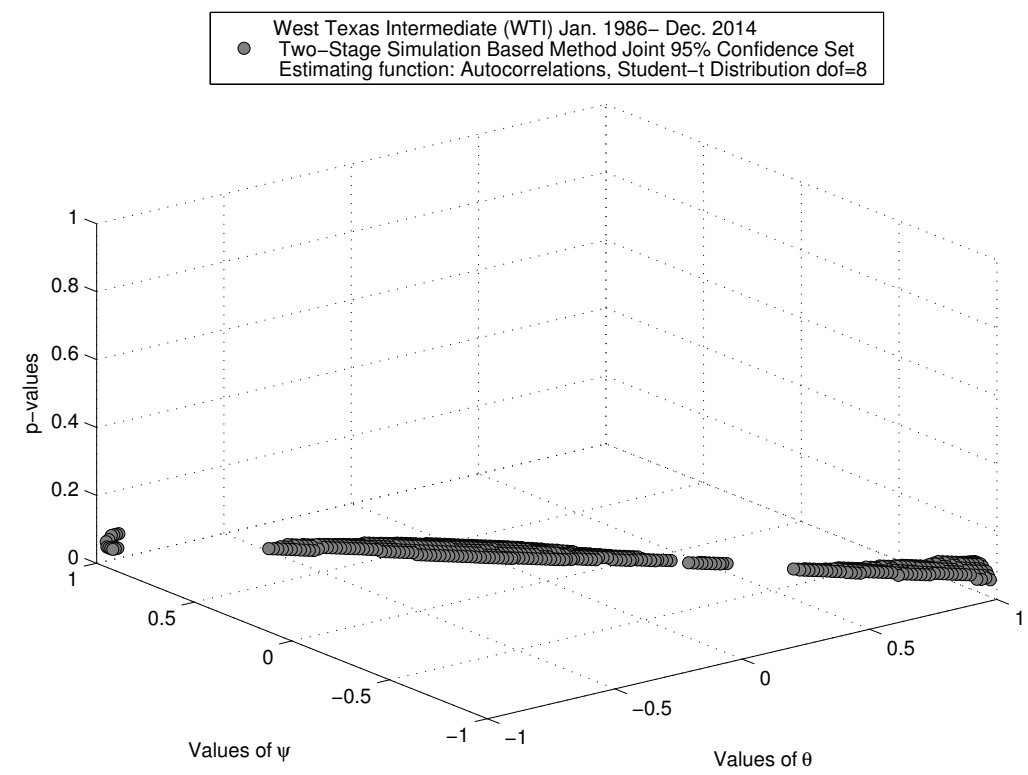


Figure (iii)

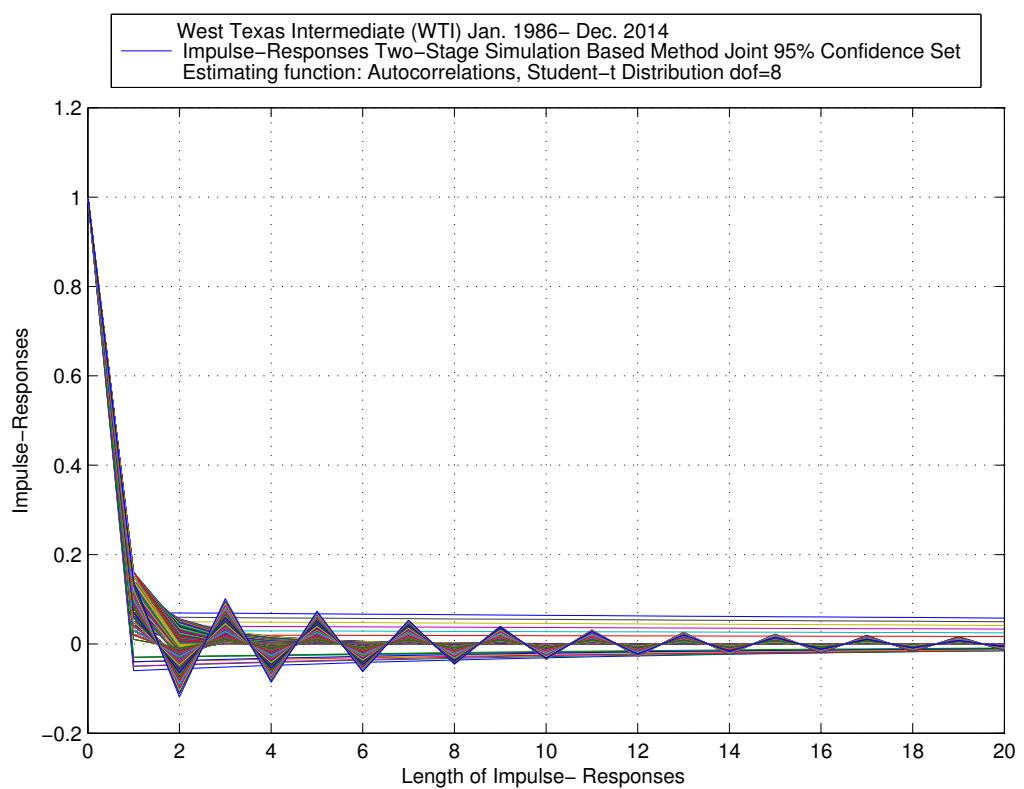

Figure (iv)

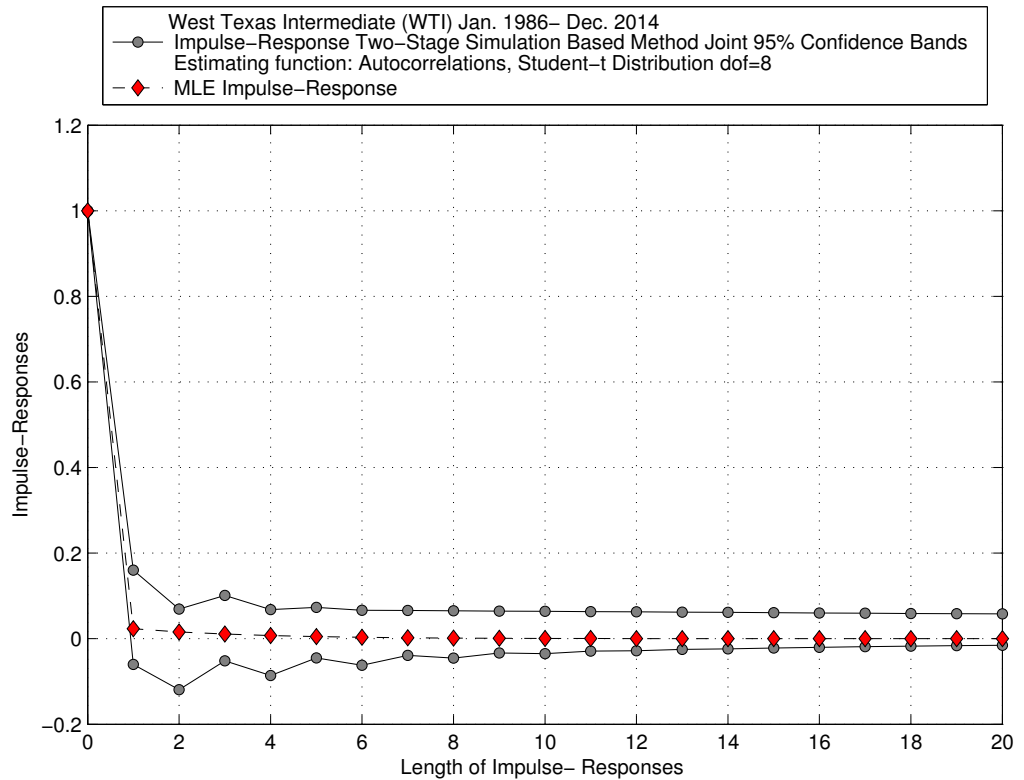


Figure 2.10: West Texas Intermediate (WTI) Jan. 2010- Dec. 2014 (Weekly). Student-t Distribution with 8 Degrees of Freedom

Figure (i)

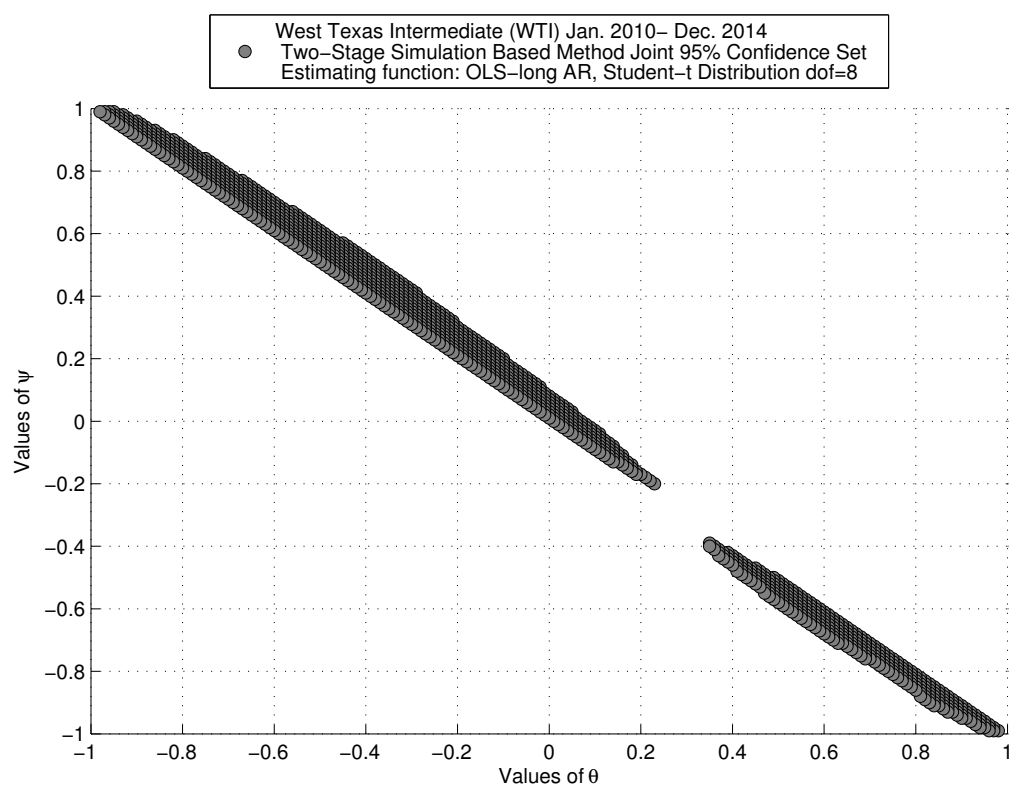

Figure (ii)

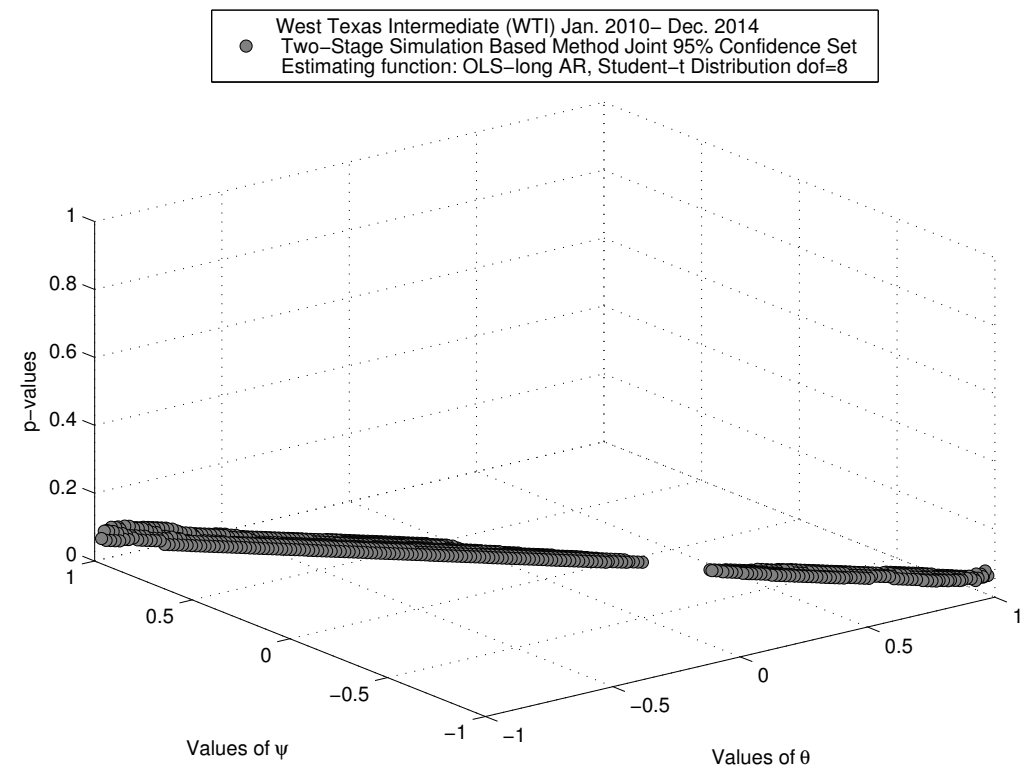


Figure (iii)

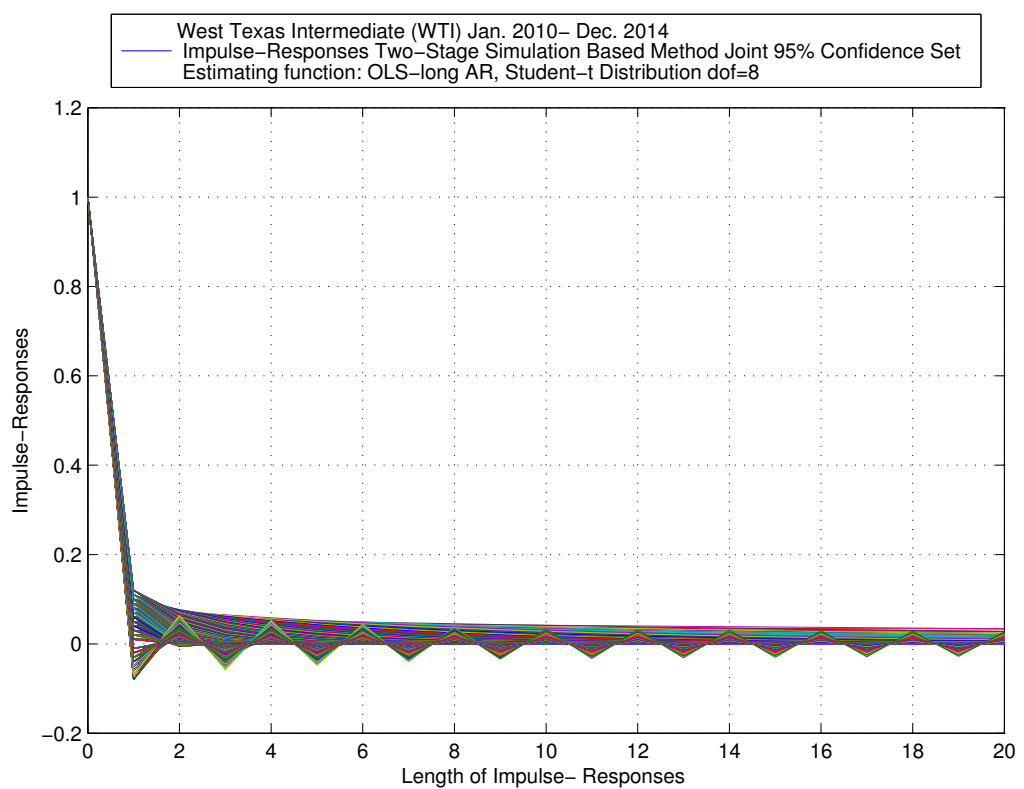

Figure (iv)

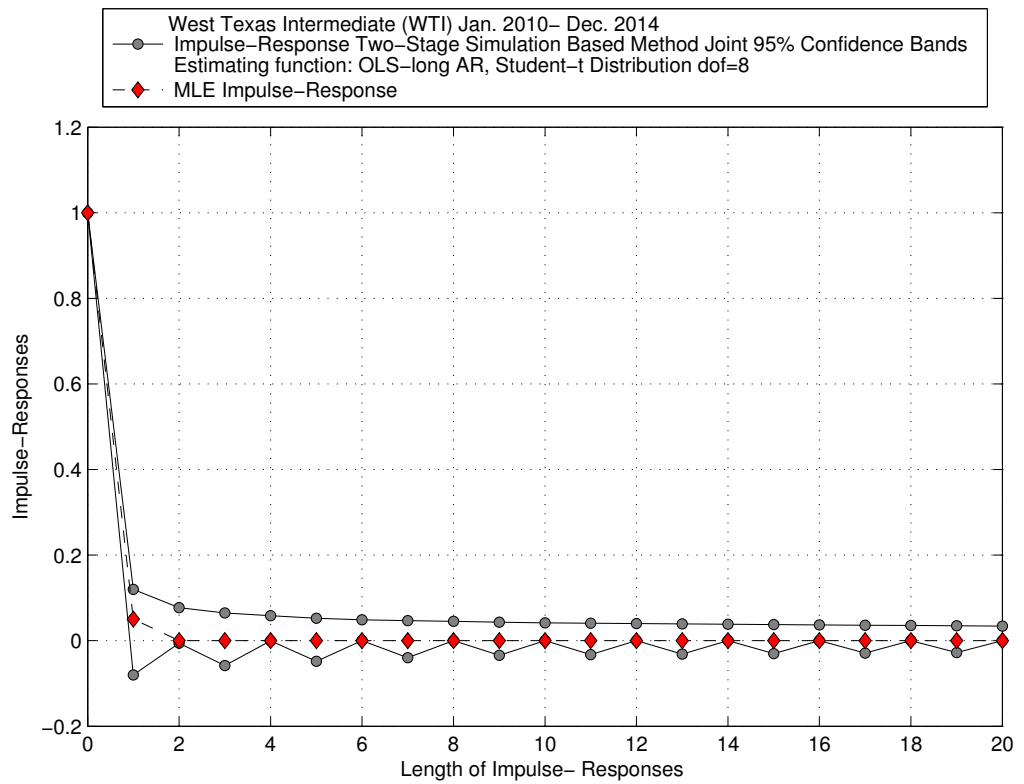


Figure 2.11: Western Canadian Select (WCS) May 2008 Dec. 2014 (Weekly). Gaussian Distribution

Figure (i)

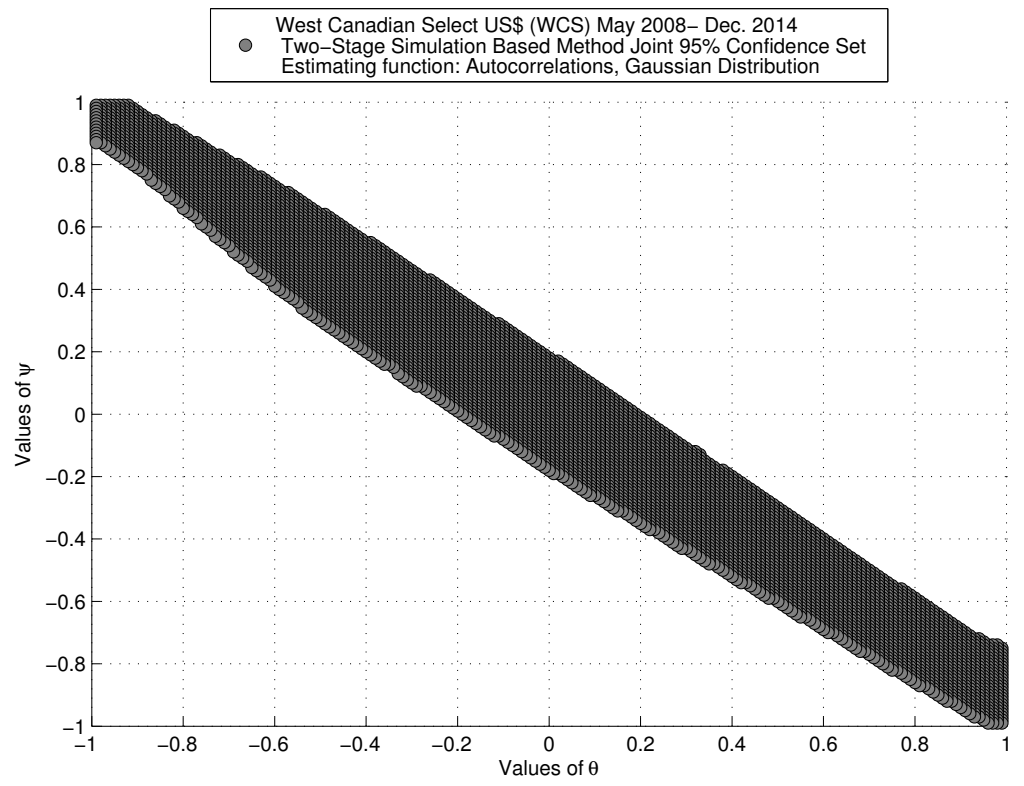

Figure (ii)

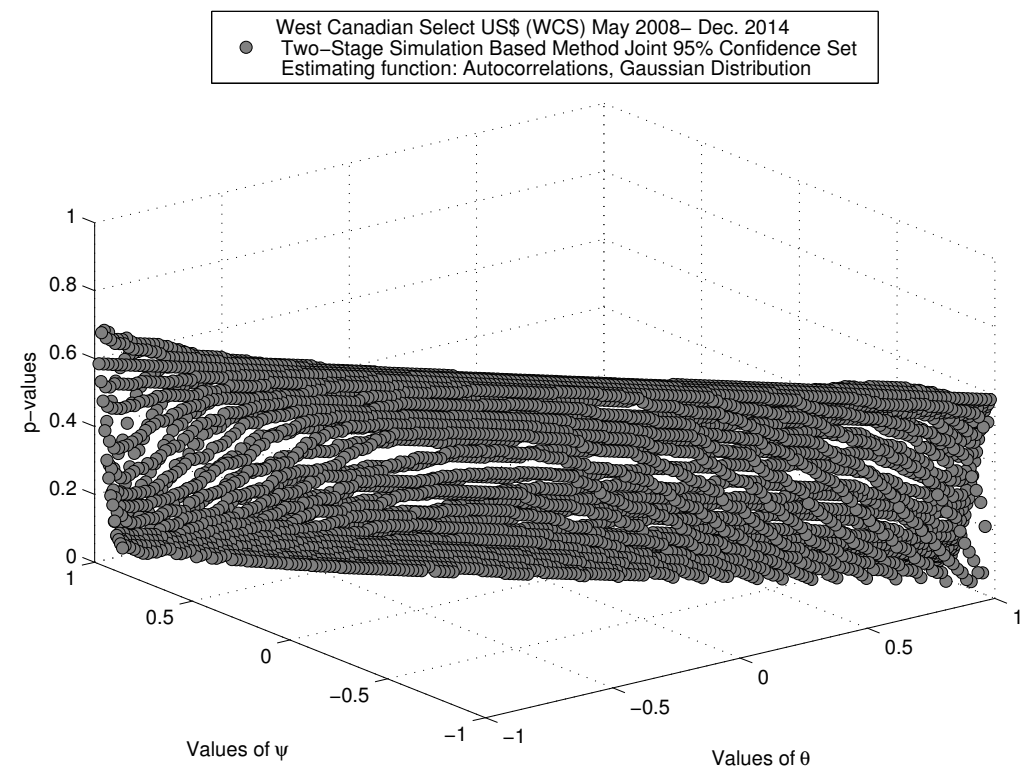


Figure (iii)

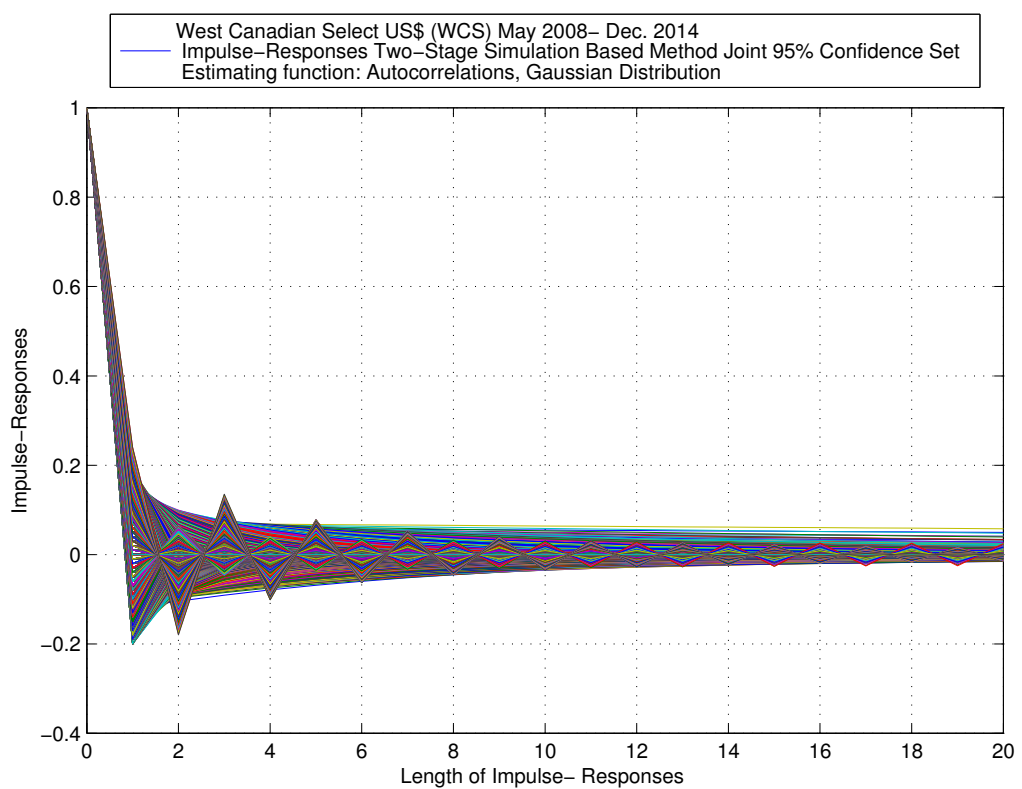

Figure (iv)

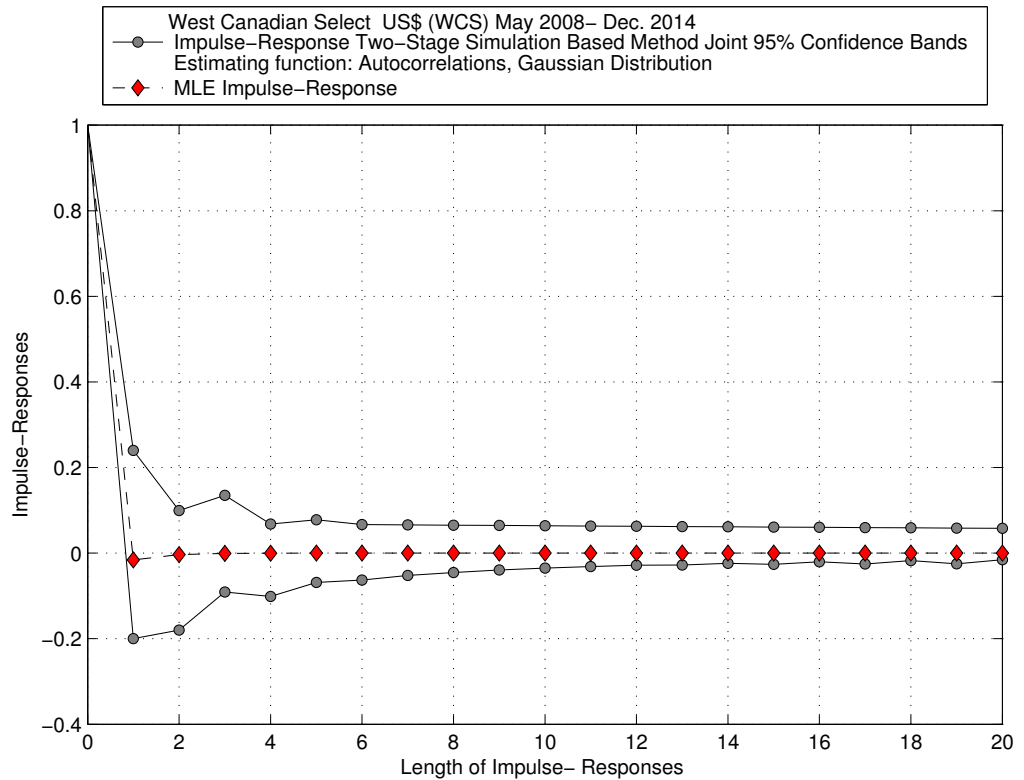


Figure 2.12: Western Canadian Select (WCS) May 2008 Dec. 2014 (Weekly). Stable Non-Gaussian Distribution

Figure (i)

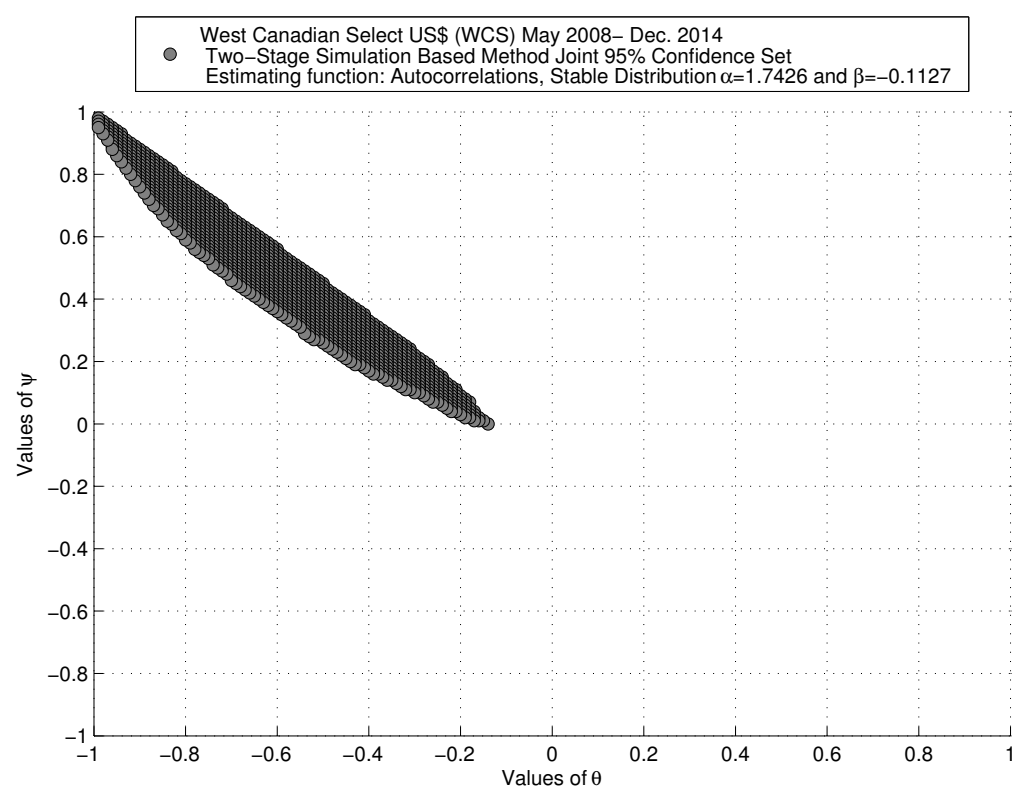

Figure (ii)

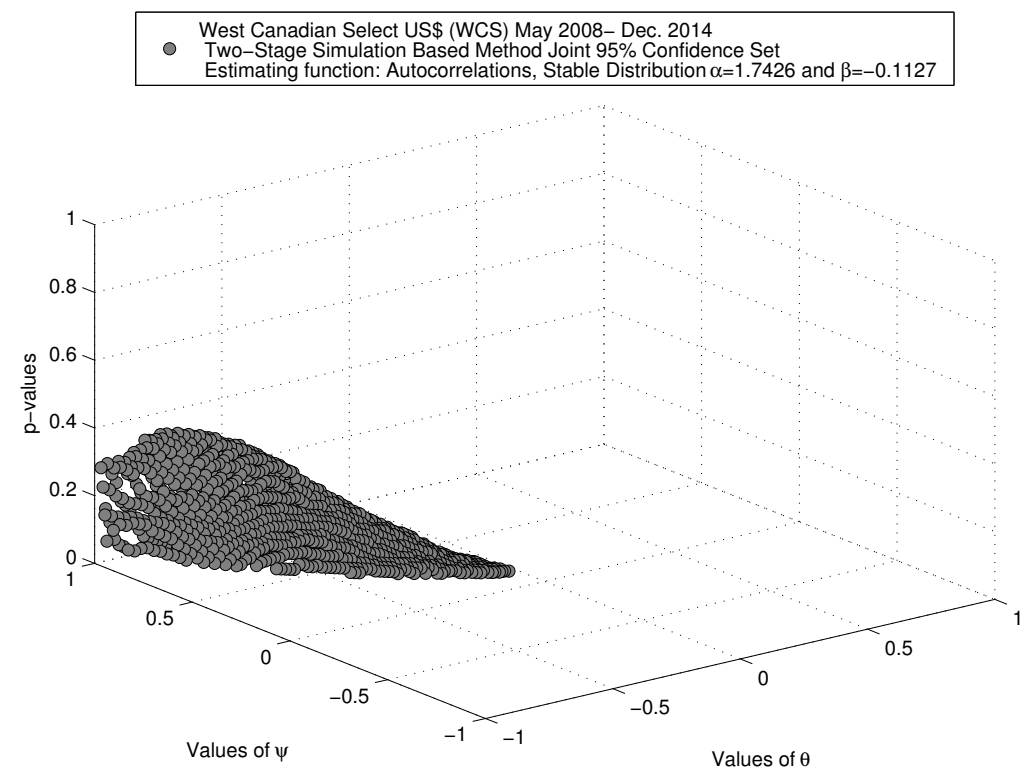


Figure (iii)

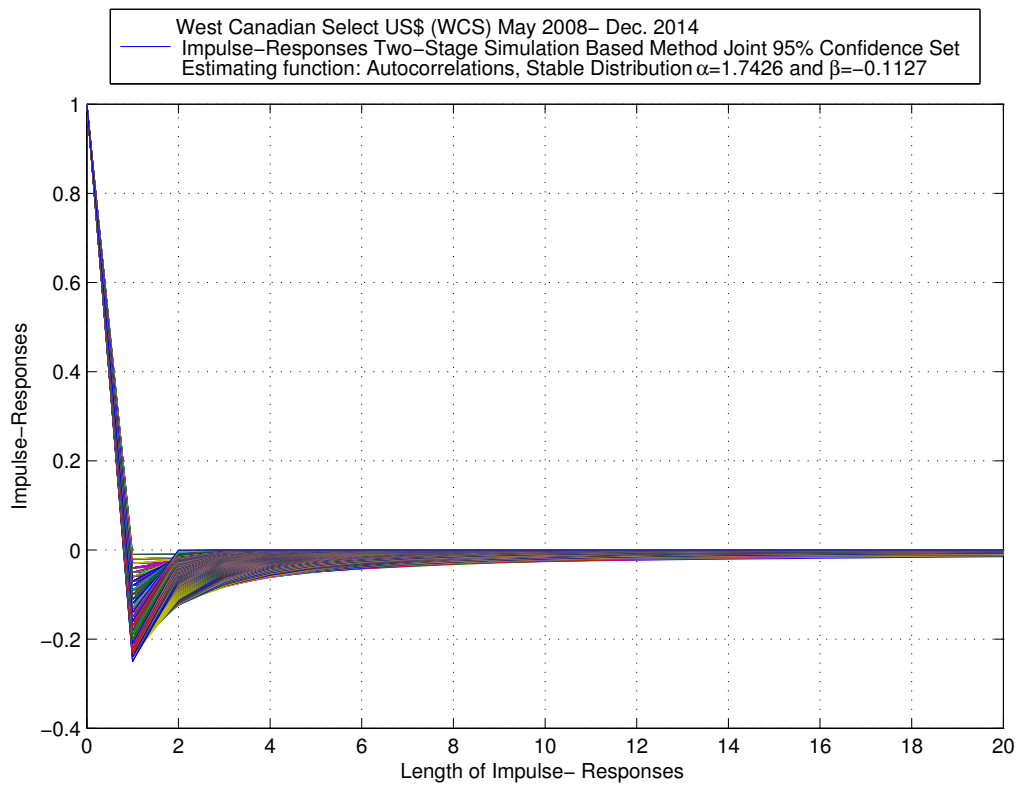

Figure (iv)

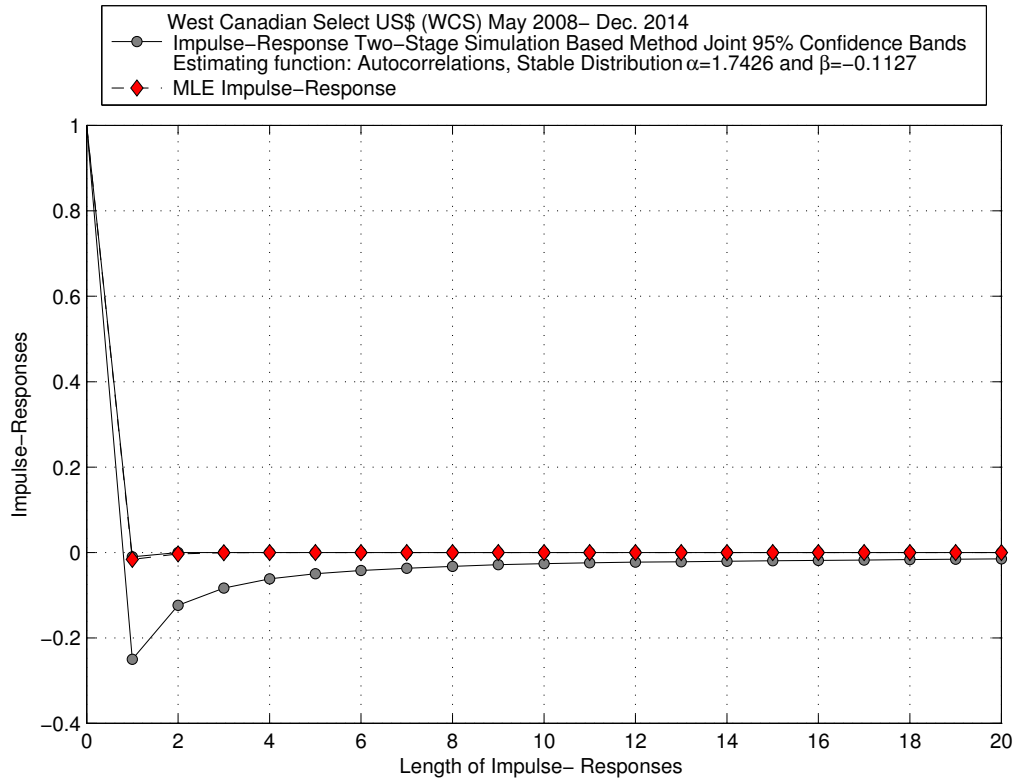


Figure 2.13: Western Canadian Select (WCS) May 2008 Dec. 2014 (Monthly). Gaussian Distribution

Figure (i)

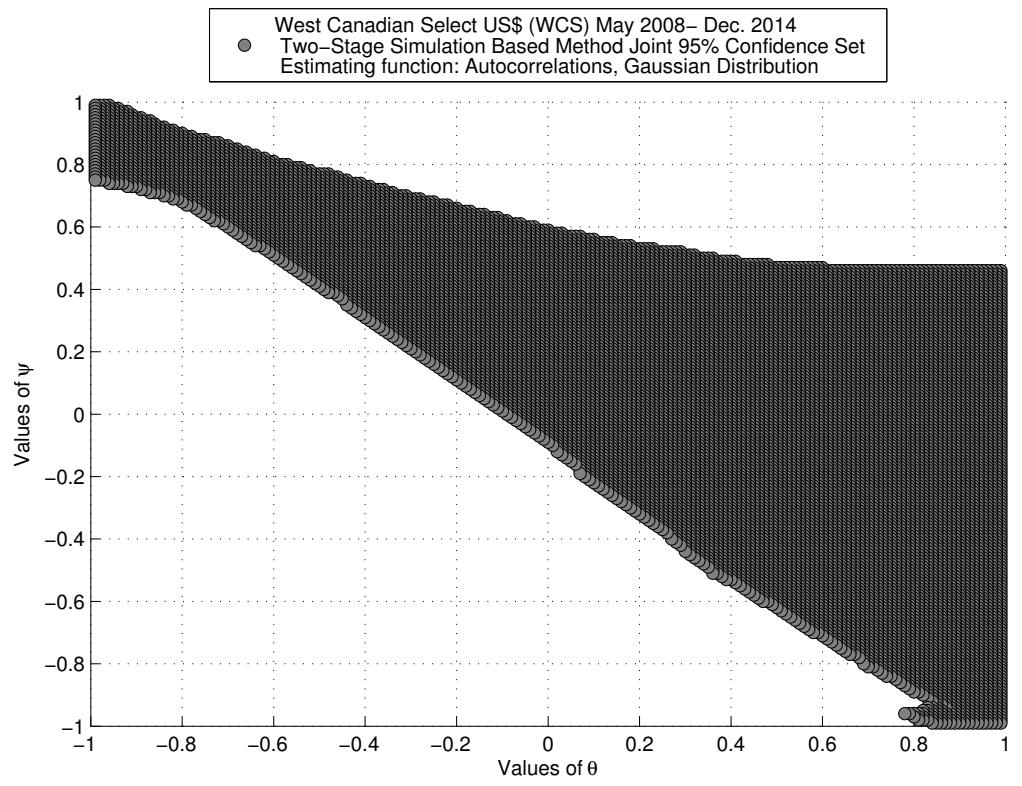

Figure (ii)

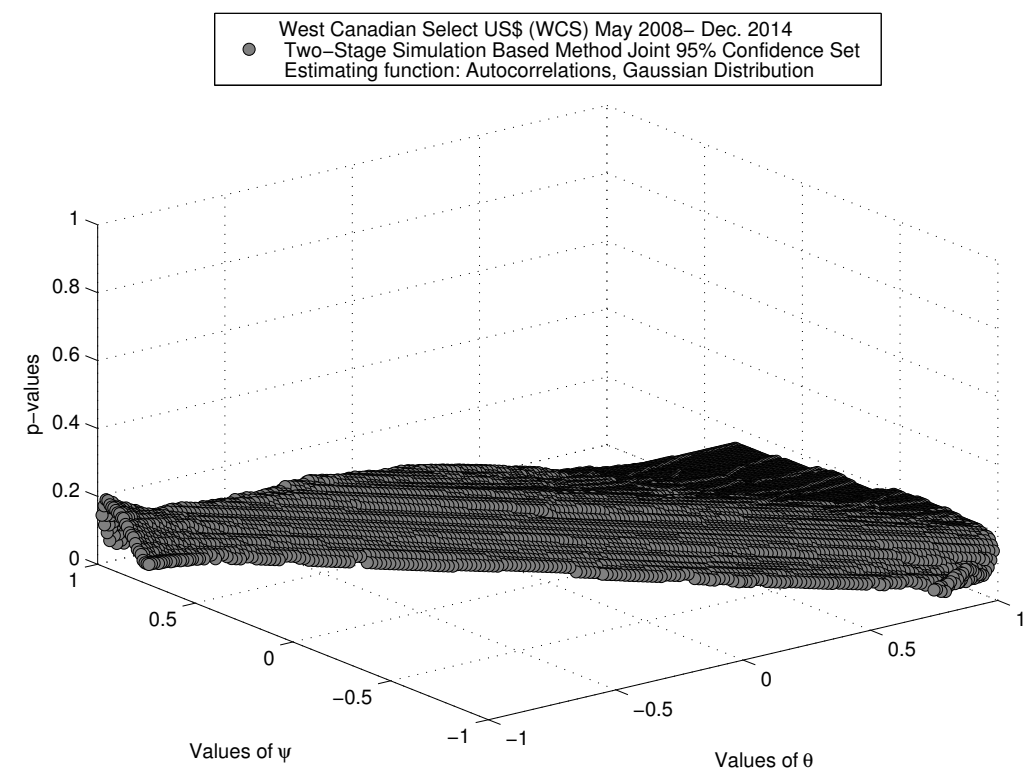


Figure (iii)

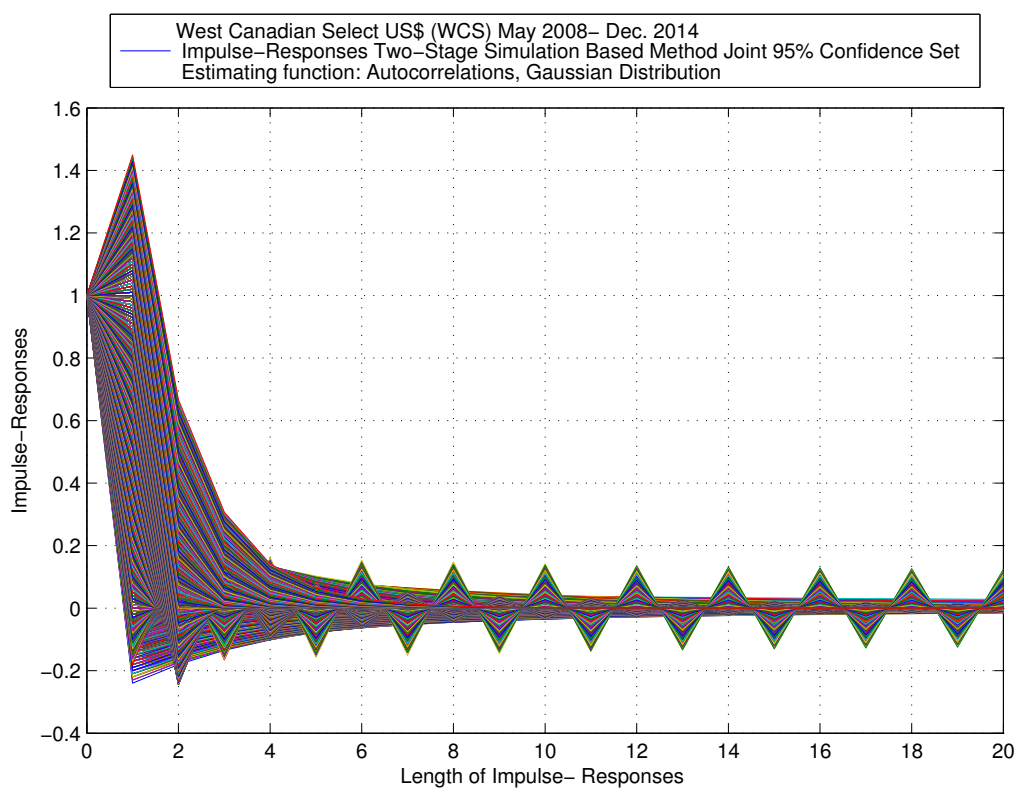

Figure (iv)

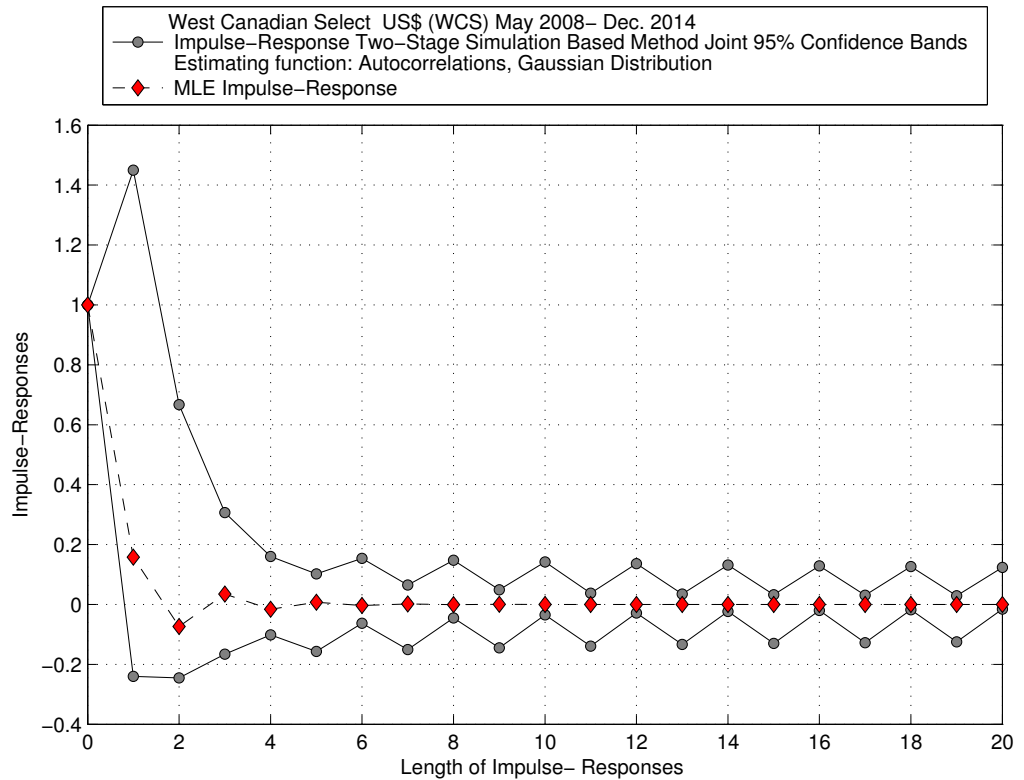


Figure 2.14: Western Canadian Select (WCS) Jan. 2010- Dec. 2014 (Weekly). Gaussian Distribution

Figure (i)

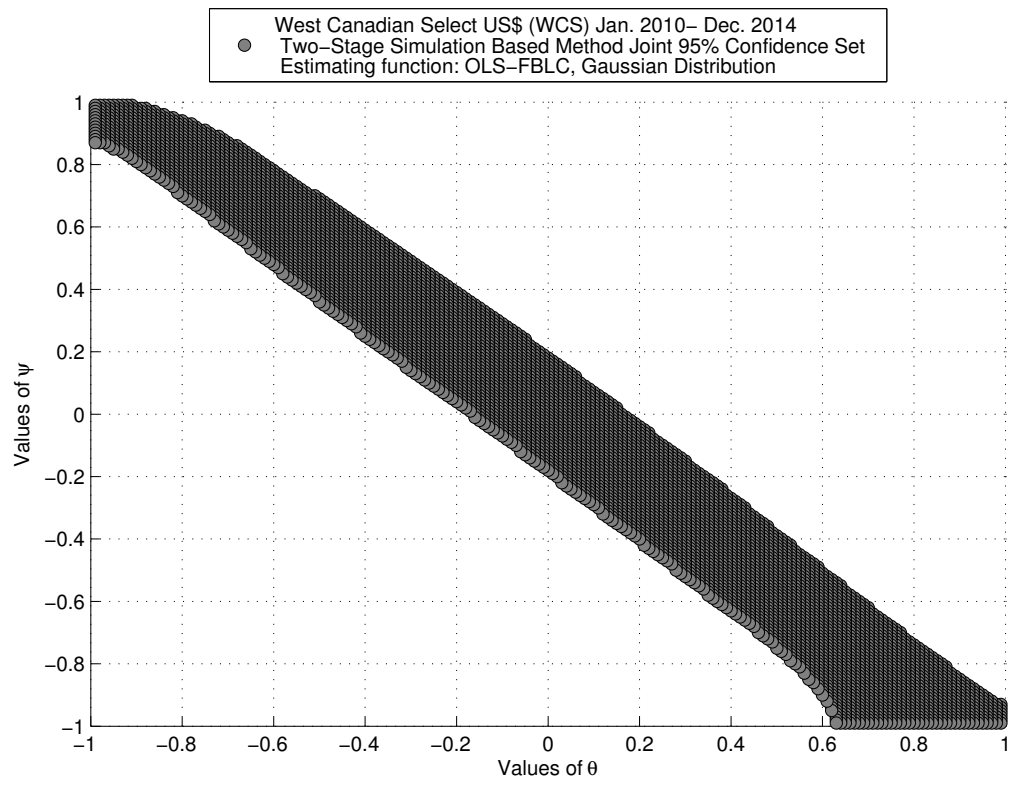

Figure (ii)

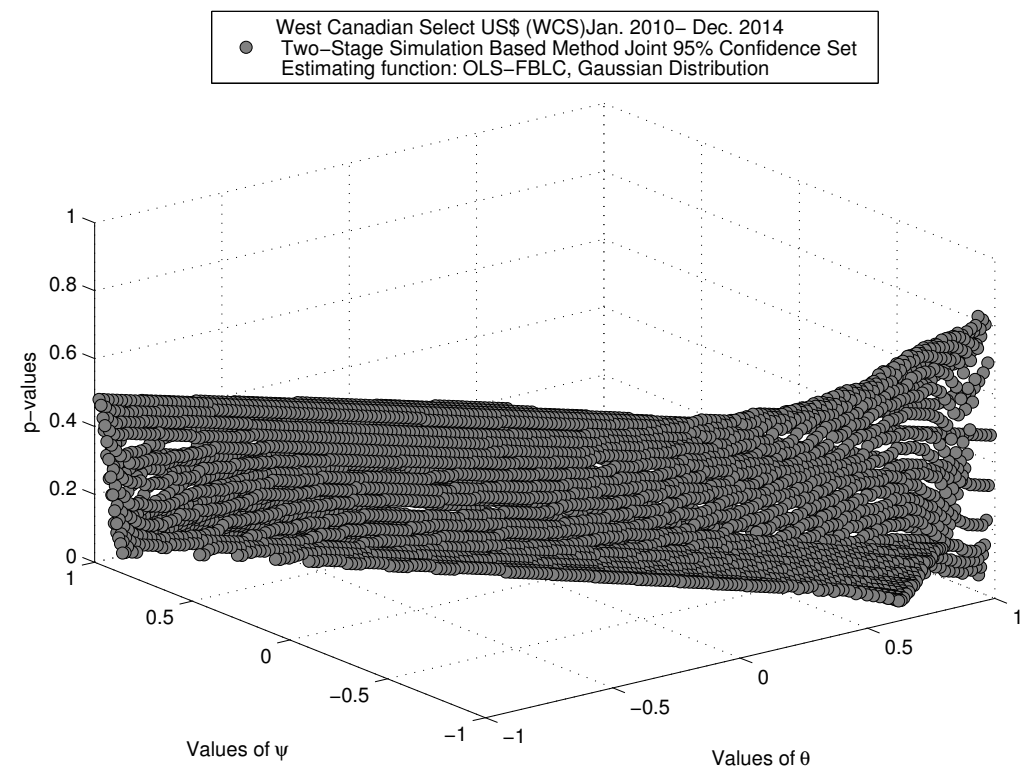


Figure (iii)

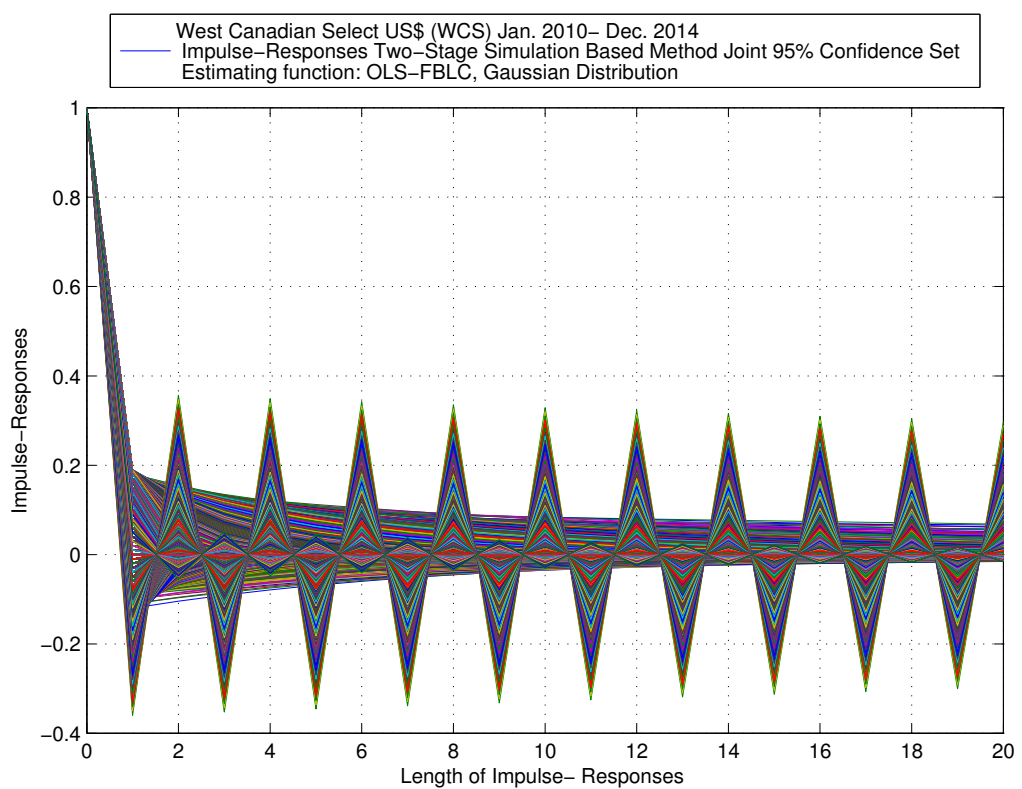

Figure (iv)

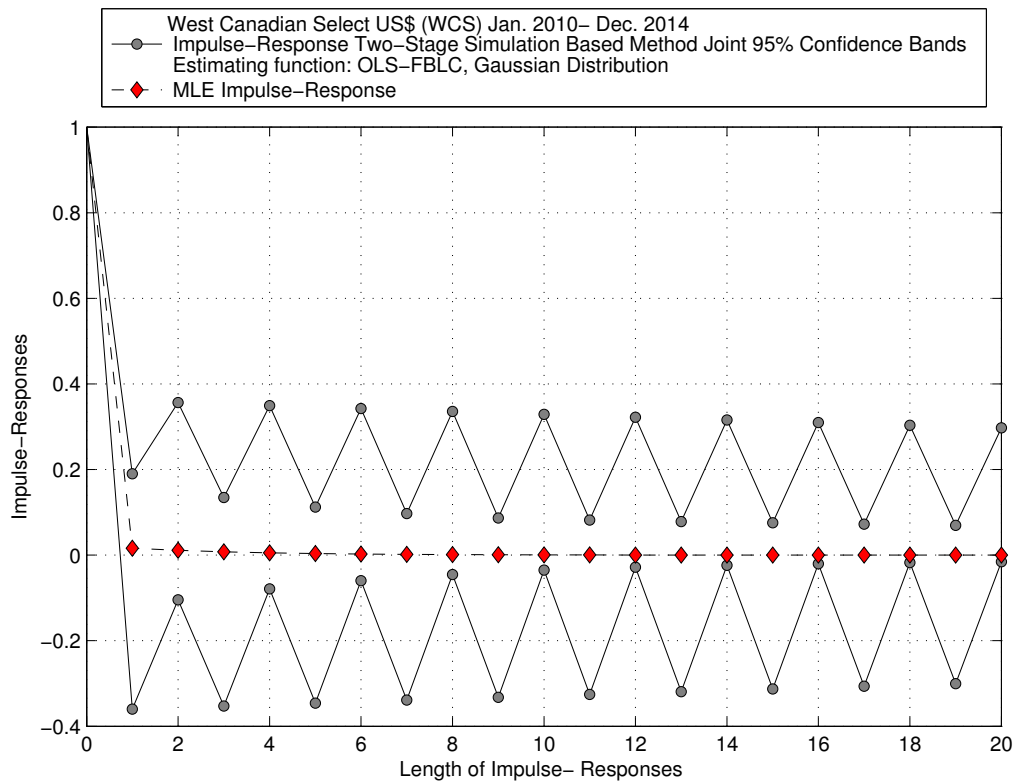




\section{Chapter 3}

\section{The Persistence of Idiosyncratic Shocks in Non-Diversifiable Portfolios}

\subsection{Introduction}

The value of a stock can be defined as the discounted value of future expected cash flows and the volatility is an indicator of the variability or risk (i.e. uncertainty) observed in the stock returns as the flow of information is processed by capital market participants. According to the modern portfolio theory (MPT) pioneered by Markowitz (1952 and 1959) risk averse investors seek to maximize expected returns considering a given level of market risk, which translates into an evaluation of stocks in terms of risk-return profiles. However, firms are exposed to both systematic and idiosyncratic risks. Systematic risks can be explained as the exposure to market and economic factors that are not diversifiable in a portfolio. Idiosyncratic risks correspond to firm-specific circumstances, such as, the presence of a new competitor, a regulatory change, management change, and product launch or recall, which are diversifiable or mitigated by adding different sectors to the portfolio. Not 
all investors hold diversified portfolios in the way the financial theory suggests. Investors may face financial constraints, or may have a concentrated position in one or few particular stocks. Hence, latent exposures to idiosyncratic risks are important determinants of stock returns to be considered for forecasting and portfolio construction purposes.

Contemporary research in finance has focused on both time series and cross sectional studies of idiosyncratic risk, looking for trends or breaks in specific periods, and correlations across markets, industries and countries. Campbell et al. (2001) introduce a Capital Asset Pricing Model (CAPM) - based estimation of aggregate idiosyncratic volatility setting the first steps in this line of research. They document a positive deterministic trend in average idiosyncratic firm- level volatility, not observed in industry or market volatility during the sample period 1962-1997 for the United States (US) market. Following the work by Campbell et al (2001), the papers by Xu and Marlkiel (2003), Bali et al. (2008), Brandt et al. (2010), Bekaert et al. (2012) and references therein, focus on the behaviour and determinants of the idiosyncratic volatility. Xu and Marlkiel (2003) analyze the US market during the years 1952 -1998 and explain that to satisfy investors appetite for growth during 1990s firms undertook investment decisions that increased on average their vulnerability to idiosyncratic risks. Bali et al. (2008) find a significant upward trend in the new measure for the period 1962-2005, but it is not as strong as the one observed with the measure of Campbell et al. (2001). However, Brandt et al. (2010) study the years 1925-2008 and find that the observed trend in the US market is reversed and by 2003 the volatility has fallen back to pre-1990 levels. In contrast to Xu and Marlkiel (2003), they suggest that the level of idiosyncratic volatility in the 1990s is an episodic phenomenon induced by retail trading (buy-and sell-small trades) and not institutional ownership. Lastly, Bekaert et al. (2012) extend the data of Campbell et al. (2001) to 2008 and find that idiosyncratic volatility can be explained by a stationary autoregressive process with regime switches of short duration, instead of a trend. They conclude that cash flow variables related to growth, market volatility, and cyclical risk drive most of the variation in idiosyncratic volatility, both in the 
United States and at the international level when they analyze data from G7 countries.

Since idiosyncratic risk is embedded in the volatility of stock market returns and not directly observable, the abovementioned studies consider different estimation methodologies. The common approach in the financial literature is to decompose stock returns to separate idiosyncratic and systematic risks. Theoretically, the idiosyncratic volatility can be estimated using the residuals from a factor model, either the well-known Capital Asset Pricing Model (CAPM), or the three-factor model from Fama and Frech (1993). Campbell et al. (2001) propose to take the residuals from the CAPM and construct weighted measures for both market and industry idiosyncratic risks. However, this estimation requires a large panel of industries and firms to be able to eliminate the covariances and factor exposures by taking weighted averages. Xu and Marlkiel (2003) use Generalized Autoregressive Conditional Heteroskedasticity (GARCH) models to estimate the conditional volatility jointly with Monte Carlo simulations for calibrations. In contrast, Bali et al. (2008) introduce a model-independent measure based on the concept of gain from portfolio diversification. This particular measure only requires the computation of the variance of a portfolio comprising a group of stocks belonging to a particular sector, the non-diversified portfolio, and the variance of the relevant market-wide index, the diversified portfolio. Lastly, Garcia et al. (2014) introduce a new model-free measure based on the cross sectional dispersion of stock returns and show that their measure has a correlation close to $90 \%$ with the one used by Goyal and Santa-Clara (2003) and above $97 \%$ for the rest the previous measures, including Bali et al. (2008). The main advantage of this latest measure over the previous monthly ones is that it can also be computed daily.

In this chapter, we propose to assess the persistence of shocks on a measure of idiosyncratic risk (MIR) for a non- diversifiable portfolio of stocks pertaining to a particular economic sector with simultaneous impulse-response confidence bands. The MIR is a residual series, which is computed following Bali et al. (2008) by deducting the variance of the market portfolio from the variance of the non-diversified portfolio. In line with the 
assumptions of the univariate GARCH models, which account for Autoregressive Moving Average, ARMA process on square returns, the MIR is expected to follow this process as well. Our framework has a built in test for the ARMA assumption, since we obtain non-empty confidence sets if this assumption is satisfied.

There is a large literature on fitting the GARCH family of models to the volatility of financial assets, originated by the work of Engle(1982) and Bollerslev (1987). The GARCH family have incorporated volatility asymetries, explained as more sensitivity to bad news or market downswings than to market upswings, with models, such as, the exponential generalized autoregressive conditional heteroskedastic (EGARCH) by Nelson (1991), and the Threshold GARCH or TGARCH by Zakoian (1994). Since the literature has identified the propagation of volatility between financial assets, research has been developed to capture symmetric and asymmetric volatility spillovers within markets as well as across markets, countries and regions. For instance, Chan et al. (1991) study volatility spillovers between the S\&P 500 index futures market and the market for the stocks underlying the index with a bivariate GARCH and impulse-responses. In addition, the multivariate GARCH family of models has been enhanced to be able to capture volatility spillovers among countries, see Li and Giles (2015). Moreover, research has gone further in an effort to fully capture volatility spillovers, for example, Diebold and Yilmaz (2009, 2012) develop a volatility spillover index based on forecast error variance decompositions from vector autoregressions; and recently, Baruník et al. (2015) have refined Diebold and Yilmaz's (2009 and 2012) approach to best capture spillover asymmetries.

Our methodology offers the advantage of isolating the idiosyncratic risk to be able to understand the persistence of shocks and spillovers at sector-specific levels with a univariate approach. Our research contributes to the literature in two ways: first, provides better understanding of inherent idiosyncratic risks and spillovers relevant for portfolio diversification; second, the output of our method can be used to rank non-diversified portfolios in terms of average resilience for risk management purposes. 
We apply the two-stage simulation based method, introduced in the first chapter, with a particular refinement that allows harvesting information from the three estimating functions. Our method delivers joint confidence regions for the parameters of $\operatorname{ARMA}(1,1)$ processes, which are projected to obtain simultaneous impulse-response confidence bands. This method contains simulations to calibrate auxiliary parameter estimates, and to compute the empirical p-values via Dufour's (2006) Monte Carlo test method. We consider three estimating functions to define the auxiliary parameters: (i) two-sided regression with forward and backward looking components (leads and lags), (ii) long-autoregression (AR) and (iii) empirical autocorrelations. An statistic corresponding to the choice of each estimating function is built measuring the distance between the estimates obtained from the data and their expected values, calibrated from the estimated computed in the first simulation stage. In addition, the average of the statistics of the three estimating functions is computed as a forth resulting test statistic to harvest information from the other three. Each statistic is inverted applying the $\mathrm{MC}$ test method by collecting the values of the parameters that are not rejected under the null hypothesis, for which the MC p-value is higher than a significance level $\alpha$, to obtain a joint confidence set. Lastly, simultaneous impulse-response confidence bands are computed on each case by projecting the joint confidence regions following the well-known recursions, which define impulse-response functions in ARMA models.

Applying econometric methods to generated measures or indexes is not unusual in the financial literature. In fact, many series available in the macroeconomic and financial literature are constructed based on prices. A well know series is the Volatility Index (VIX), introduced in 1993 by the Chicago Board Options Exchange (CBOE), computed as a weighted average of S\&P 500 stock index option prices designed to reflect a consensus view of short term expected stock market volatility. The VIX is used in various studies as proxy for market uncertainty or investors expectations; for instance, in the estimation of Fama and French augmented factor model by Durand et al. (2011), and the recent research by Bekaert and Hoerova (2014), which decompose the squared VIX index into conditional variance of 
stock returns and equity variance premium, and evaluate the predictive power of the VIX and its components for stock market returns, economic activity and financial instability.

The empirical illustration describes the construction of a non-diversified portfolio containing the common stocks of the three major Telecom carriers in Canada trading in the Toronto Stock Exchange. The Telecom sector in Canada is regulated, hence the stock volatility of public carriers is affected by the following sector-specific factors: product market interaction including technological developments, government regulation, and the entrance or intentions to enter in the Canadian market by local and foreign firms as a result of changes in regulation (for instance, spectrum auctions). Moreover, all these factors shape the perception of the future risk performance of the three firms considered. The period of study comprises 10 years of market data, from 2005 to 2014, and the measure of interest is computed on a monthly frequency. It is an active period in the Canadian Telecom market due to changes in government regulations including spectrum auctions, and sector-specific events, for instance, the deployment of new technology supporting high data rates jointly with wireless voice services. In addition, market-wide events, such as, the international financial crisis and the Great Recession occurred during the years 2007-2009.

The results obtained can be summarized as follows. A standard linear detrending method is applied in order to separate the short-term behaviour of the MIR and study the persistence of shocks. The two-stage simulation method is applied considering both Gaussian and Student-t distributions. Since the outcome obtained with the Student-t distributions is close to that of the Gaussian, but gives larger confidence sets and has more coverage of the white noise pair, the Gaussian distribution is preferred. As expected, our method delivers closed confidence sets for the ARMA parameters in all cases and shows robustness to root cancellation, or common roots in AR and MA polynomials, explained in detail in the first chapter. The behaviour of our impulse-reponse confidence bands is compared with that of the impulse-response functions produced by the MLE method. We find that MLE underestimates the persistence of shocks, since it shows a small impact with no 
feedback effects, which disappear on the second month. In contrast, our impulse-response confidence bands are able to capture the presence of various effects. The impulse-response confidence bands obtained with AR-based estimating functions capture shocks with large and persistent oscillatory feedback effects, which are symmetric. However, the impulse response confidence bands computed with the autocorrelations estimating function describe shocks with a short-lived major impact and relatively small oscillatory feedback effects, which show minor asymmetries. In fact, the presence of oscillatory effects is the result of pairs containing a large negative AR root. As the AR root increases, reaching values higher than the MA root in absolute value and getting closer to the unit root, the oscillatory effects become more persistent; in contrast, as the AR root decreases and becomes smaller than the MA root in absolute value, the oscillatory effects tends to disappear. The pattern found on the impulse-response functions with the averaged statistic is in line with the one obtained with the autocorrelations estimating function. I conclude that the effect of a shock on the MIR for the Canadian Telecom portfolio can be described as a large, but short-lived initial impact with small oscillatory feedback effects that remain close to zero after the eight month. This result indicates that these firms are able to contain the transitory uncertainty and spillover effects to keep their idiosyncratic risk at manageable low levels.

The remaining sections of this chapter are organized as follows. Section 3.2 presents the estimation of the MIR and the description of our methodology, including main assumptions and estimation of impulse-response confidence bands. Section 3.3 provides a description of the data. The results are discussed in Section 3.4 and Section 3.5 presents our concluding comments. 


\section{$3.2 \quad$ Framework}

\subsubsection{Idiosyncratic Risk}

The common approach in the financial literature is to decompose stock returns to isolate idiosyncratic and systematic risks. The return of an asset can be defined as a linear combination of two components, one random component corresponding to the aggregate risk and one idiosyncratic component. A pure individual idiosyncratic process is defined as a shock uncorrelated with aggregate risk and uncorrelated with shocks to other assets, for instance a Gaussian white noise process with zero mean and finite variance. In contrast, the covariance between the return of two assets is explained as a measure capturing the co-movements between shocks, largely market-wide, and shocks that affect each individual asset. Thus, if assets corresponding to a particular subset of the economy are collected, for example, an industry or sector, a large part of the risks faced by the members of this subset is highly correlated, including the presence of both systematic risks, and group- specific or idiosyncratic risks. The objective of computing the MIR is to capture a process that is uncorrelated with market-wide shocks, which reflects both firm and sector latent factors.

Following Bali et al. (2008), a model- independent measure can be computed as follows. A non-diversified portfolio is assembled with $\mathrm{N}$ assets pertaining to a particular sector. The expected return of this portolio $r_{P, t}$ and its variance $\sigma_{P, t}^{2}$ are computed below, where $r_{i, t}$ represents the excess return of a stock $i$ with respect to a risk free rate; $\sigma_{i, t}$ is the standar deviation of excess returns, and $\rho_{i, j}$ is the correlation of excess returns between stocks $i$ and $j$ :

$$
\begin{gathered}
r_{P, t}=\sum_{i=1}^{N} w_{i, t} r_{i, t}, \\
\sigma_{P, t}^{2}=\sum_{i=1}^{N} w_{i, t}^{2} \sigma_{i, t}^{2}+2 \sum_{i=1}^{N} \sum_{j>i}^{N} w_{i, t} w_{j, t} \sigma_{i, t} \sigma_{j, t} \rho_{i, j} .
\end{gathered}
$$


It is assumed that there are not short-sales, so seach weight $w_{i, t}$ is positive and the sum of weights satisfy $\sum_{i=1}^{N} w_{i, t}=1$.

As it is well known in the modern portfolio theory (MPT) pioneered by Markowitz (1952 and 1959) diversification is obtained by adding stocks with low correlation among them, to offset movements in idiosyncratic risk that affect the value of the stocks. The variance of a fully diversified portfolio reflects only the market or systematic risks because there are diversification gains of including a number of stocks that are less than perfectly positively correlated. Bali et al. (2008) assume the excess returns are perfectly correlated, $\rho_{i, j}=1$, so no diversification gains are achieved. As a result, the variance of the non-diversifed portfolio is simplified to the value-weighted average standard deviations of excess returns in equation (3.2.3), which contains both systematic and idiosyncratic risks:

$$
\sigma_{P, t}^{2}=\left(\sum_{i=1}^{N} w_{i, t} \sigma_{i, t}\right)^{2} .
$$

The stock market is identified as a fully diversified portolio, due to the diversification gains of including a large number of stocks, and $\sigma_{M, t}^{2}$, the variance of the excess returns of the market index represents pure systematic risk,

$$
\sigma_{M, t}^{2}=\operatorname{Var}\left(r_{M, t}\right)
$$

Lastly, the proposed MIR, represented by $\mathcal{M}_{t}^{2}$ is computed by deducting the variance of the market portfolio $\sigma_{M, t}^{2}$, from the variance of the non-diversified portfolio:

$$
\mathcal{M}_{t}^{2}=\sigma_{P, t}^{2}-\sigma_{M, t}^{2}
$$




\subsubsection{Assumptions}

The data abstracted from the presence of a trend follows a zero mean $\operatorname{ARMA}(1,1)$ process of size $T$, with unknown parameters $\theta_{0}$ and $\psi_{0}$,

$$
\mathcal{M}_{t}^{2}=\psi \mathcal{M}_{t-1}^{2}+e_{t}+\theta e_{t-1}, \quad t=1, \ldots, T
$$

with

$$
e_{t}=\sigma u_{t}
$$

where $|\theta|<1$ guarantees invertibility or an Autoregressive $\operatorname{AR}(\infty)$ representation, $|\psi|<1$ ensures stationarity, the joint distribution of $u_{1}, \ldots, u_{t}, \ldots, u_{T}$ is known and $\sigma$ is a nuisance parameter which is partialled out once the two-stage simulation-based method is applied. For instance, $u_{t}$ can be assumed independent and identically distributed with a Gaussian distribution:

$$
u_{t} \stackrel{i . i . d .}{\sim} \mathcal{N}(0,1), \quad t=1 \ldots T .
$$

We assume that $X_{0}=e_{0}$, where $e_{0}$ is fixed. In addition, Student-t errors are considered.

An exact test is formulated for the following joint null hypothesis,

$$
H_{0}\left(\theta_{0}, \psi_{0}\right): \theta=\theta_{0} \text { and } \quad \psi=\psi_{0},
$$

which is applied by collecting pairs satisfying (3.2.9) to construct a joint confidence set.

Along the following sections, we will analyze the behaviour of the MIR, isolate the short-run behaviour of the series and estimate the persistence of shocks via the application of our two-stage simulation-based method.

\subsubsection{Two-Stage Simulation-Based Method}

Our method follows the Indirect Inference methodology, which assumes the existence of an approximate or auxiliary model to describe the data. The complete description of the 
methodology is contained in the first chapter of this thesis.

We can define the estimation of the auxiliary model over the data vector $\mathcal{M}$ as

$$
\hat{\lambda}=\hat{\Lambda}(\mathcal{M})
$$

where $\hat{\Lambda}($.$) is the estimating function. The auxiliary parameter \hat{\lambda}$ can be obtained via three estimating functions: (i) OLS estimation with forward-looking and backward-looking components (leads and lags),(ii) OLS estimation of a long-autocorrelation (AR), and (iii) empirical autocorrelations. We refer to these choices as:

i.

$$
\hat{\Lambda}_{f b}(\mathcal{M})=\underset{\gamma}{\operatorname{argmin}} Q(\mathcal{M}), \quad \text { with } \quad \gamma=\left(\gamma_{f}^{\prime}, \gamma_{b}^{\prime}\right)^{\prime}
$$

ii.

$$
\hat{\Lambda}_{b}(\mathcal{M})=\underset{\gamma_{0}}{\operatorname{argmin}} Q(\mathcal{M}), \quad \text { with } \quad \gamma_{0}=\left(0, \gamma_{b}^{\prime}\right)^{\prime}
$$

where

$$
\begin{array}{r}
Q(\mathcal{M})=\sum_{t=p+1}^{T}\left[\mathcal{M}_{t}-\gamma_{f 1} \mathcal{M}_{t+1}-\ldots-\gamma_{f p} \mathcal{M}_{t+p}\right. \\
\left.-\gamma_{b 1} \mathcal{M}_{t-1}-\ldots-\gamma_{b p}^{*} \mathcal{M}_{t-p}\right]^{2}
\end{array}
$$

with $\gamma_{f}=\left(\gamma_{f 1}, \ldots, \gamma_{f p}\right)^{\prime}, \gamma_{b}=\left(\gamma_{b 1}, \ldots, \gamma_{b p}\right)^{\prime}$; and

iii.

$$
\hat{\Lambda}_{c}(\mathcal{M})=\left(\hat{\rho}_{1}, \ldots, \hat{\rho}_{p}\right),
$$

where

$$
\hat{\rho}_{j}=\frac{\hat{\operatorname{Cov}}\left(\mathcal{M}_{t} \mathcal{M}_{t-1}\right)}{\hat{\operatorname{Var}}\left(\mathcal{M}_{t}\right)} \quad j=1 \ldots p
$$

These estimating functions satisfy the scale invariance property, which allow us to evacuate $\sigma$, as showed in the proof presented in the first chapter. 
The simulation-based counterpart, given values of the parameters of interest $\beta$, is derived by creating $\mathrm{H}$ simulated paths, each of the same size as the data, applying the same estimation function on each path:

$$
\hat{\lambda}_{h}(\beta)=\hat{\Lambda}\left(x_{h}(\beta)\right) \quad h=1, \ldots, H .
$$

The estimators obtained are calibrated by computing their average over all the paths:

$$
\Lambda(\beta)=\frac{1}{H} \sum_{h=1}^{H} \hat{\lambda}_{h}(\beta) .
$$

A test statistic is constructed considering the distance betwen the previously estimated $\hat{\lambda}$ and the simulation-calibrated $\Lambda(\beta)$ auxiliary parameters for each choice of estimating function,

$$
S(\beta, \mathcal{M})=(\hat{\lambda}-\Lambda(\beta))^{\prime} \mathcal{I}_{p}(\hat{\lambda}-\Lambda(\beta))
$$

Note that the p-dimensional identity weighting matrix $\mathcal{I}_{p}$ is chosen to preserve the invariance to scale property. Any alternative choice of weighting matrix should be able to preserve this property.

A fourth test statistic is computed by averaging the statistics computed with each estimating function:

$$
\mathbf{S}_{A}(\beta, \mathcal{M})=\frac{\sum_{i=1}^{3} S_{i}(\beta, \mathcal{M})}{3} .
$$

Next we apply the MC method from Dufour (2006) to compute the p-values and introduce the test inversion. This is done by adding a new layer of simulation and drawing $L$ replications for each value of $\beta_{0}$ satisfying the null hypothesis, regardless of the value of $\sigma$. Hence, for each replication $l$ we obtain the auxiliary estimates,

$$
\hat{\lambda}_{l}\left(\beta_{0}\right)=\hat{\Lambda}\left(\mu_{l}\left(\beta_{0}\right)\right)
$$


applying the same estimating function as before, and compute a series of statistics for each choice of estimating function:

$$
S_{l}\left(\mu_{l}\left(\beta_{0}\right)\right)=\left(\tilde{\lambda}_{l}\left(\beta_{0}\right)-\Lambda\left(\beta_{0}\right)\right)^{\prime} \mathcal{I}_{p}\left(\tilde{\lambda}_{l}\left(\beta_{0}\right)-\Lambda\left(\beta_{0}\right)\right)
$$

These statistics are similar to the one in equation (3.2.18), but take the vector of estimated parameters $\hat{\lambda}_{l}\left(\beta_{0}\right)$ instead of the data-based one. The exchangeability property of the MC test permits using the same vector of calibrated parameters in both statistics.

Consequently, by the averaging method we obtain a series of statistics as:

$$
\mathbf{S}_{A, l}\left(\mu_{l}\left(\beta_{0}\right)\right)=\frac{\sum_{i=1}^{3} S_{i, l}\left(\mu_{l}\left(\beta_{0}\right)\right)}{3} .
$$

The empirical $p$-value associated with the null hypothesis is computed as:

$$
\hat{p}\left(\beta_{0}\right)=\frac{G_{L}+1}{L+1}
$$

where $G_{L}$ denotes the number of times the statistic $S_{l}\left(\mu_{l}(\beta)\right)$ is greater than or equal to the data-based statistic $S(\beta, \mathcal{M})$.

Out of all the pairs tested, we collect those values not rejected by the test, for which the empirically computed $p$-value, $\hat{p}\left(\beta_{0}\right)$, is higher than the confidence level $\alpha$. Hence, we are able to obtain exact $p$-values for finite samples. As a result, we obtain a joint projectionbased confidence set for the $\operatorname{ARMA}(1,1)$ parameters in question satisfying equation (3.2.9) denoted $C S\left(\beta_{0} ; \alpha\right)$, which satisfies the following inequality:

$$
P[\beta \in C S(\beta ; \alpha)] \geq 1-\alpha .
$$

This confidence region can take various shapes: (i) a closed interval, (ii) unbounded intervals, (iii) the entire real line, and (iv) an empty set. If we obtain a closed interval, we can conclude that the parameters of interest are well identified. However, if we have unbounded 
confidence sets, either unbounded intervals or the entire real line, our method suffers from a partial or complete identification problem. Obtaining an empty confidence set indicates a rejection of the null hypothesis for any pair, and, as a result, a rejection of the empirical counterpart of the binding function selected, and the $\operatorname{ARMA}(1,1)$ specification as a whole.

The empirical impulse-response functions, denoted $g_{i}(\beta)$ for $i=1, \ldots, m$, are obtained by projecting the joint confidence region for well identified parameters. This implies minimizing and maximizing $g_{i}(\beta)$ over the values in $C S\left(\beta_{0} ; \alpha\right)$ and applying the image function $g_{i}(C S(\beta ; \alpha))$ to satisfy the following inequality:

$$
P\left[g_{i}(\beta) \in g_{i}(C S(\beta ; \alpha)), i=1, \ldots, m\right] \geq 1-\alpha .
$$

\subsection{Data}

A non-diversify portfolio is constructed considering the common stock of the three major nationwide Telecom carriers in Canada trading in the Toronto Stock Exchange: Telus Corporation (T), Rogers Communications Inc. (RCI. B) and BCE Incorporated (BCE). The historical series of daily closing prices and market capitalization of the three stocks, and daily closing prices of the S\&P TSX Composite Index (considering trading dates and excluding holidays) are retrieved from Bloomberg, from January 4, 2005 to December 31, 2014. The Bank of Canada 1-month Treasury-bill yield for the same period is obtained from Statistics Canada CANSIM Table 176-0048 and adjusted using 365 days. Daily returns are

computed as $r_{t}=\ln \left(\frac{P_{t}}{P_{t-1}}\right)$, where $P_{t}$ and $P_{t-1}$ represent daily prices at times $t-1$ and $t$, respectively. The excess returns are obtained by deducting the 1-month Treasury-bill yield from the computed returns.

A summary of statistics of excess returns in presented in Table 3.1, followed by histograms and kernel density estimates in Figures 3.1 to 3.4 . 
Table 3.1: Excess Returns: Summary of Statistics

\begin{tabular}{|l|c|c|c|c|c|}
\hline \multicolumn{1}{|c|}{ Excess Returns } & Obs. & Mean & Std.Dev. & Skewness & Kurtosis \\
\hline BCE (BCE) & 2510 & 0.000197 & 0.015414 & -8.028203 & 226.153560 \\
\hline Rogers (RCI.B) & 2510 & 0.000381 & 0.015889 & -0.149529 & 9.044791 \\
\hline Telus (T) & 2510 & 0.000296 & 0.014577 & -0.262591 & 13.577904 \\
\hline S\&P TSX Composite & 2510 & 0.000141 & 0.011912 & -0.709248 & 13.606247 \\
\hline
\end{tabular}

As expected, the excess returns of the S\&P TSX Composite Index, called the Index henceforth, have the lowest mean and standard deviation. Among the stocks, Rogers' excess returns have the highest mean and standard deviation. All excess returns are negatively skewed and display higher kurtosis than the Gaussian density. The high level of kurtosis indicates that historical excess returns are more clustered around zero and the negative skewness points out that big changes in returns or outliers are found mostly on the left tail.

The skewness and kurtosis of BCE's excess returns in Figure 3.2 are the highest in absolute value. In particular, BCE's large skewness is expected due to the joint effect of the financial crisis and a failed leveraged buyout attempt by a private equity consortium, which caused large negative returns in November 2008. ${ }^{1}$ All figures display unimodal densities with heavy tails, which are common findings in the financial literature.

A study of the correlations among the excess returns of the three stocks and the Index, not presented here for brevity, permits to analyze empirically which factors lead the behaviour of these returns in particular periods of time. During the period of study, the correlations between each stock excess returns are close to $40 \%$ on average. Once various samples are considered, we find that during the period comprising the financial crisis and the Great Recession, the average correlation of the stocks excess returns with the Index excess returns is the highest, over $50 \%$. However, during the years following this period, the correlations between the three stocks excess returns are $10 \%$ higher than the correlations between the stocks and the Index's excess. In particular the correlations between the three

\footnotetext{
${ }^{1}$ In terms of squared deviations from the mean return, the return of this particular event is the largest contributor to the stock variance of that particular month.
} 
stocks excess returns reach $70 \%$ in 2014 , which can be related to the uncertainty generated by the expectations that a foreign wireless carrier could enter the Canadian market. Since this uncertainty is idiosyncratic, it is safe to assume that it is completely diversified awayby the composition of the Index. ${ }^{2}$

The monthly variance of the three stocks and the Index is computed as squared deviations of daily excess returns $r_{s, d}$ with respect to its mean $\bar{r}_{s, d}$, considering the number of trading days on each month $D_{t}$ :

$$
\sigma_{s, t}^{2}=\sum_{d=1}^{D_{t}}\left(\frac{r_{s, d}-\bar{r}_{s, d}}{D_{t}-1}\right) \quad \text { for } s=1, . ., 4
$$

The variance of the non-diversified portfolio is computed as the square of the weighted average standard deviation of the three stocks excess returns, as explained in equation (3.2.5). There are arguments in the literature supporting the use of both, market capitalization and equal weights. In this study, the variance of the non-diversified portfolio was compared considering both alternatives. ${ }^{3}$ The monthly market capitalization value for each stock is computed as the average of the daily values of each month. The monthly weights are computed as the monthly market capitalization value for each stock divided over the sum of the market capitalizations of the three stocks. The average monthly weights over the 2005- 2014 period for the three stocks are: BCE $43.36 \%$, Rogers $31.42 \%$ and Telus $25.22 \%$. The market capitalization values of $\mathrm{BCE}$ and Rogers reflect their asset mix, which includes broadcasting assets that have a larger value for BCE. Yet, we find that the measure based on market capitalization weights in this case gives results that are in line with the outcomes obtained with the measure based on equal weights, despite giving a small bias toward BCE. Hence, the following sections focus on the measure computed using equal weights.

\footnotetext{
${ }^{2}$ The proportion of the Telecom sector on the Index is $4.81 \%$, which also includes the stock of the local carrier Manitoba Telecom Services Inc. (MBT). The highest weights on the S\&P TSX Composite Index correspond to the Financial sector 34\%, followed by the Energy sector $20.83 \%$ and the Materials sector $11.54 \%$.

${ }^{3}$ Garcia et al. (2014) show that the equal-weighted cross-sectional variance is a consistent and asymptotically efficient estimator for idiosyncratic variance. Hence, this estimator dominates the value-weighted one.
} 
The variance of the Index is deducted from the variance of the non-diversified portfolio to construct the measure of idiosyncratic risk (MIR). As mentioned above, the objective is to isolate the idiosyncratic risk from the systematic risk; hence the MIR represents on average the idiosyncratic risk dynamics including spillovers effects. MIR's descriptive statistics are shown in Table 3.2. We have a small sample with 120 monthly observations, and the MIR's high kurtosis and positive skewness indicate that the observations are more clustered around zero and that extreme values are mostly found on the right tail. These characteristics are expected for measures based on squared returns as described by Andersen et al. (2001) and references therein. The measure computed using market capitalization weights shows notably higher skewness and kurtosis than the measure with equal weights, as a result of the mentioned bias towards BCE.

Table 3.2: Measure of Idiosyncratic Risk: Summary of Statistics

\begin{tabular}{|l|c|c|c|c|c|}
\hline Series & Obs. & Mean & Std. Dev. & Skewness & Kurtosis \\
\hline MIR & 120 & 0.000076 & 0.000226 & 2.743554 & 37.3795 \\
\hline
\end{tabular}

Figure 3.5 displays the MIR considering an equal-weighted variance of the non-diversified portfolio. As a result of its construction, MIR's observations are mostly on the positive side. The underlying assumption in the methodology is that the variance of the nondiversified portfolio is larger than the variance of the Index, since its main components are the sector and firms idiosyncratic risks. Thus, positive values of the MIR indicate points in time when the variance of the non-diversified portfolio is greater than the variance of the Index, or greater idiosyncratic risk, and negative values indicates that the variance of the Index or market or systematic risk is greater. In particular, the MIR shows one large extreme value on the market side, reflecting the market crash in October 2008 during the international financial crisis, and another one on the idiosyncratic side, corresponding to the failed acquisition attempt mentioned before that affected BCE's idiosyncratic risk in November 2008. 


\subsection{Results}

Traditional statistical tests are conducted to examine the presence of a trend on the MIR series considering the original series and a series with the two major outliers downweighted. First, the Dickey-Fuller Generalized Least Squares (DF-GLS) unit-root test is conducted to have an estimate of the optimal lag selection. Then, the Augmented Dickey-Fuller (ADF) unit-root is applied based on the number of lags selected before, since the DF-GLS test is well known to have greater power than the later. In addition, the Phillips -Perron test is implemented allowing the default lag selection with the Newey-West standard errors to account for the serial correlation. The null hypothesis of a unit-root is rejected for the three tests considering a 0.05 level of significance for the MIR, when one lag is included in the regression, no matter whether the trend and constant are included, and irrespective of whether the two major outliers in Figure 3.5 are downweighted. Lastly, the Kwiatkowski, Phillips, Schmidt, and Shin (KPSS) unit-root test fails to reject the null hypothesis that the series is trend stationary. Hence, a deterministic trend model is estimated by Ordinary Least Squares (OLS), on which the trend is significant at 0.05 level, is available upon request.

The persistence in the volatility of stocks returns is vastly described in the time series literature, for instance in GARCH models by Andersen et al. (2001) and references therein. The MIR, abstracted from the presence of a trend, is expected to follow an $\operatorname{ARMA}(1,1)$ process. To be able to describe the persistence of idiosyncratic shocks on the MIR, we apply the two-stage simulation-based method and compare the the impulse-response confidence bands with the impulse-response functions obtained via MLE point estimation. The shortcomings of MLE are well known in the literature in terms of identification problems with small samples as described in previous chapters. In contrast, our method is identification robust and achieves size control and good power for small samples.

The setting of the two-stage simulation-based method is the following. The total number of auxiliary parameters is set to 8 , which in terms of the estimating functions means: (i) 8 parameters in total in the OLS estimation with forward and backward looking components, 
or FBLC for shortness, (ii) 8 parameters in the OLS estimation of the long autoregression, or long-AR and (iii) 8 empirical autocorrelations. The number of paths in the simulation step is set to 3 , and we conduct a grid search over the four quadrants with a step of 0.01 . An identity weighting matrix is used to preserve the invariance to scale of the statistic associated to every choice of the estimating function. Lastly, we select 199 replications in the MC test method to compute the empirical p-values, once the test inversion is introduced, and consider a level of significance $\alpha=0.05$. As output of our method we obtain four diagrams: joint $95 \%$ confidence set, confidence surface, the projected impulse-response functions and the corresponding impulse response confidence bands.

In all the studies considering Gaussian and Student-t distributions with 5 and 8 degrees of freedom I obtain closed confidence sets for the three estimating functions, for which large positive values of the MA parameter $\theta$ correspond to large negative values of the AR parameter $\psi$, and vice versa. The results obtained with the Student-t distributions are closer to the Gaussian, but we obtain larger confidence sets and more coverage of the white noise pair. As a result, the Gaussian distribution is preferred. The results obtained with the Student-t distributions are briefly described and available upon request. In addition, our method shows robustness to root cancellation in all cases. The MLE impulse-response function based on point estimates shows a small impact with no feedback effects, which disapear on the second period. In contrast, our impulse-response confidence bands are able to capture the presence of various effects which are explained along the following lines.

When the Gaussian distribution is assumed, the confidence sets computed on the detrended MIR series with the FBLC and long-AR estimating functions have similar shapes, including pairs on the second, third and four quadrants. However, the confidence set obtained with the FBLC estimating function extends further on the second and fourth quadrants. These confidence sets do not contain the pair $\left\{\theta_{0}, \psi_{0}\right\}=\{0,0\}$, which gives a white noise process. In contrast, the confidence set obtained with the autocorrelations estimating function includes points in the four quadrants, with more points located on the second and 
third quadrants and it contains the white noise pair. The impulse-response confidence bands computed with both long-AR and FBLC estimating functions contain sharp parallel oscillatory behaviours, which are more pronounced for the FBLC. The presence of oscillatory effects is the result of pairs containing a large negative AR root. As the AR root increases, reaching values higher than the MA root in absolute value and getting closer to the unit root, the oscillatory effects become more persistent; in contrast, as the AR root decreases and becomes smaller than the MA root in absolute value, the oscillatory effects tends to dissapear. The impulse-response confidence bands computed with the autocorrelations estimating function show a large but short-lived major impact and relatively small feedback effect with small parallel oscillatory behaviours. The diagrams presented in Figures 3.6 and 3.7 correspond to the outcome obtained with the long-AR and Autocorrelation estimating functions: the $95 \%$ confidence set appears first, followed by the corresponding confidence surface and the impulse-response functions; lastly the confidence bands, plotted together with the MLE impulse-response function, are presented on the last figure. Once the averaging method is considered, the confidence set and the pattern found on the impulse-response functions is in line with the one obtained with the autocorrelations estimating function. The outcome of the averaging method is shown in Figure 3.8. The impulse-response confidence bands are able to capture the persistence of the MIR, which remains close to zero after the eigth month approximately.

The confidence sets obtained with the Student-t distribution include more pairs on the first and second quadrants. In particular, the confidence set obtained with the long-AR estimating function assuming a Student-t with 5 degrees of freedom has one area with a similar shape to the one of the confidence set obtained with the long-AR estimating function and Gaussian distribution, and another small disjoint area on the upper left of the second quadrant. The confidence sets obtained with both, the long-AR and autocorrelations estimating functions with the Student-t distribution with 8 degress of freedom include the white noise pair. As expected, the impulse-response confidence bands for the three esti- 
mating functions and degrees of freedom assumptions behave similarly to the ones obtained with the Gaussian distribution, including the ones computed with the averaging method.

\subsection{Conclusion}

The persistence of shocks on the measure of idiosyncratic risk (MIR) for a non-diversified portfolio of Canadian Telecom stocks is assessed with impulse-responses confidence bands by applying the two-stage simulation-based method introduced in the first chapter of this thesis. The MIR is computed following Bali et al. (2009), relying on modern portfolio theory (MPT) to isolate the idiosyncratic risk from the systematic risk. A standard detrending method is applied to the MIR to be able to capture its short term behaviour. The two-stage simulation-based method delivers joint confidence regions for the parameters of the $\operatorname{ARMA}(1,1)$ processes, which are projected to obtain simultaneous impulse-response confidence bands. Our framework has a built in test for the ARMA assumption, since we obtain non-empty confidence sets if this assumption is satisfied. Three estimating functions are considered to define the auxiliary parameters: (i) two-sided regression with forward and backward looking components (leads and lags), or FBLC, (ii) long-autoregression, or long-AR and (iii) empirical autocorrelations. Apart from the three statistics constructed based on these estimating functions, a forth statistic is proposed by an averaging method to harvest information from the other three. The simultaneous impulse-response confidence bands are obtained by projecting the joint confidence regions, which are the result of inverting the proposed statistics. The two-stage simulation-based method is applied considering both Gaussian and Student-t distributions. Since the results obtained with the Student-t distributions are closer to the Gaussian, but show larger confidence sets and more coverage of the white noise pair, the Gaussian distribution is preferred. The results indicate that the MLE impulse-response function underestimates the effect of shocks on the detrended MIR. However, our method is able to capture the presence of large and persistent oscillatory 
feedback effects with the AR-based estimating functions as a result of pairs of parameters containing a large negative AR root. The averaging method produces impulse-reponse confidence bands that are in line with the ones obtained with the autocorrelation estimating function, showing a short-lived major impact and relatively small oscillatory feedback effects, which remain close to zero after the eight month. We can conclude that the average method is able to describes the effect of a shock on the MIR for the Canadian Telecom portfolio, since these firms are able to contain the transitory uncertainty and spillover effects to keep their idiosyncratic risk at manageable low levels. 


\section{References}

[1] Andersen, Torben G., Tim Bollerslev, Francis X. Diebold, and Heiko Ebens (2001). The Distribution of Stock Return Volatility. Journal of Financial Economics 61, 43-76.

[2] Bali, T. G., N. Cakici and H. Levy (2008). A Model-Independent Measure of Aggregate Idiosyncratic Risk. Journal of Empirical Finance 15, 878896.

[3] Baruník J., Evžen Kočenda and Lukáš Vácha (2015). Asymmetric Connectedness on the U.S. Stock Market: Bad and Good Volatility Spillovers. Journal of Financial Markets 27, 55-78.

[4] Bekaert, G., Robert J. Hodrick, and Xiaoyan Zhang (2012). Aggregate Idiosyncratic Volatility. Journal of Financial and Quantitative Analysis 47, 1155-1185.

[5] Bekaert, G. and Marie Hoerova (2014). The VIX, The Variance Premium and Stock Market Volatility. Journal of Econometrics 183, 181-192

[6] Bloomberg L.P. (2015). Daily closing prices and market capitalization for Telus Corporation (T), Rogers Communications Inc. (RCI. B) and BCE Incorporated (BCE) Jan/.4/2005-Dec./31/2014. Daily closing prices of S\&P TSX Composite Index Jan/.4/2005-Dec./31/2014. Bloomberg Electronic Database. Industry Canada, C.D. Howe Building 235 Queen Street Ottawa, Ontario K1A 0H5, Canada, Jan./30/2015.

[7] Bollerslev, T. (1987). A Conditionally Heteroskedastic Time Series Model For Speculative Prices and Rates of Return. Review of Economics and Statistics 69, 542-547. 
[8] Brandt, Michael W. , Alon Brav, John R. Graham and Alok Kumar (2010). The Idiosyncratic Volatility Puzzle: Time Trend or Speculative Episodes? The Review of Financial Studies 23, 863-899

[9] Campbell, J. Y., M. Lettau, B. G. Malkiel and Y. Xu (2001). Have Individual Stocks Become More Volatile? An Empirical Exploration of Idiosyncratic Risk. Journal of Finance 56, 143.

[10] Chicago Board Options Exchange (2015). CBOE Volatility Index (VIX). Retrieved from http://www.cboe.com/micro/vix/.

[11] Diebold, F.X. and K. Yilmaz (2009). Measuring Financial Asset Return and Volatility Spillovers, with Application to Global Equity Markets. Econ Journal 119, 158-171

[12] Diebold, F.X. and K. Yilmaz (2012). Better to Give than to Receive: Predictive Directional Measurement of Volatility Spillovers International Journal of Forecast 28, $57-66$.

[13] Dufour J.-M. (2006). Monte Carlo Tests with Nuisance Parameters: A General Approach to Finite-Sample Inference and Nonstandard Asymptotics in Econometrics. Journal of Econometrics 133, 443-478.

[14] Durand, R., Dominic Lim and Kenton Zumwalt (2001). Fear and the Fama-French Factors. Financial Management 40, 409-426.

[15] Engle, R. (1982). Autoregressive Conditional Heteroscedasticity with Estimates of the Variance of United Kingdom Inflation. Econometrica 50, 987-1007.

[16] Fama, Eugene F. (1970). Efficient Capital Markets: A Review of Theory and Empirical Work. The Journal of Finance 25, 383-417.

[17] Fama, Eugene F. and Kenneth R. French (2004). The Capital Asset Pricing Model: Theory and Evidence. Journal of Economic Perspectives 18, 25-46. 
[18] Garcia, R., D. Mantilla-Garca and L. Martellini (2014). A Model-Free Measure of Aggregate Idiosyncratic Volatility and the Prediction of Market Returns. Journal of Financial and Quantitative Analysis 49, 1133-1165.

[19] Li,Y. and D.E. Giles (2015). Modelling Volatility Spillover Effects Between Developed Stock Markets and Asian Emerging Stock Markets International, Jounal of Finance and Economics 20, 155-177.

[20] Markowitz, H. (1952). Portfolio selection. Journal of Finance 7, 7791.

[21] Markowitz, H. (1959). Portfolio Selection: Efficient Diversification of Investments. Wiley, New York.

[22] MATLAB 8.1, The MathWorks, Inc., Natick, Massachusetts, United States.

[23] Nelson, D.B.(1991).Conditional Heteroskedasticity in Asset Returns: A New Approach. Econometrica 59, 347-370.

[24] StataCorp. 2011. Stata Statistical Software: Release 12. College Station, TX: StataCorp LP.

[25] Xu, Y and B.G. Malkiel (2003). Investigating the behavior of idiosyncratic volatility. Journal of Business 76, 613-45.

[26] Zakoian, J.-M. (1994).Threshold Heteroskedastic Models. Journal of Economic Dynamics and Control 18, 931-955. 
Figure 3.1: S\&P TSX: Histogram and Kernel Density Estimate

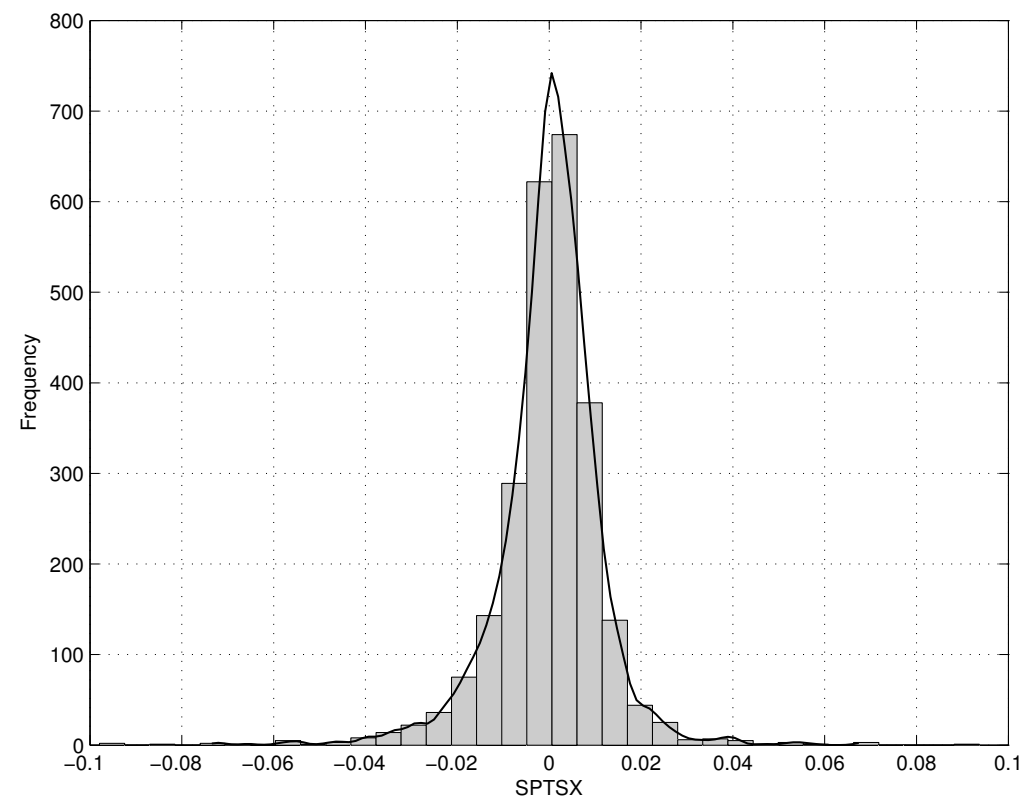

Figure 3.2: BCE (BCE): Histogram and Kernel Density Estimate

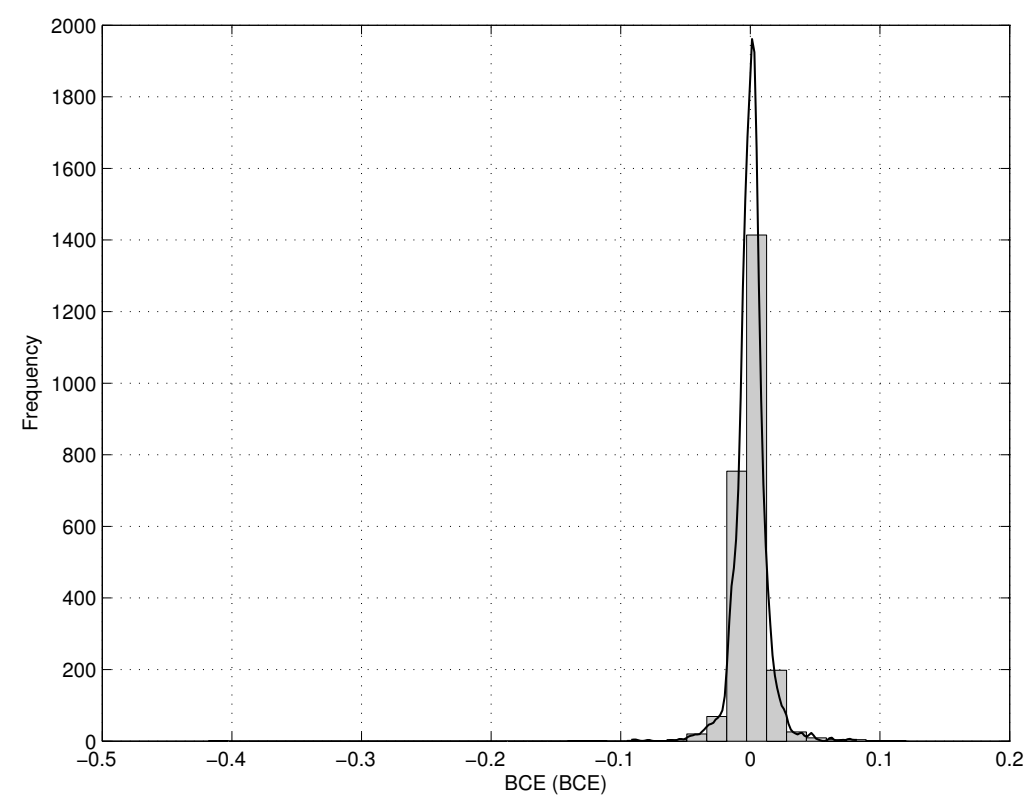


Figure 3.3: Rogers (RCI.B): Histogram and Kernel Density Estimate

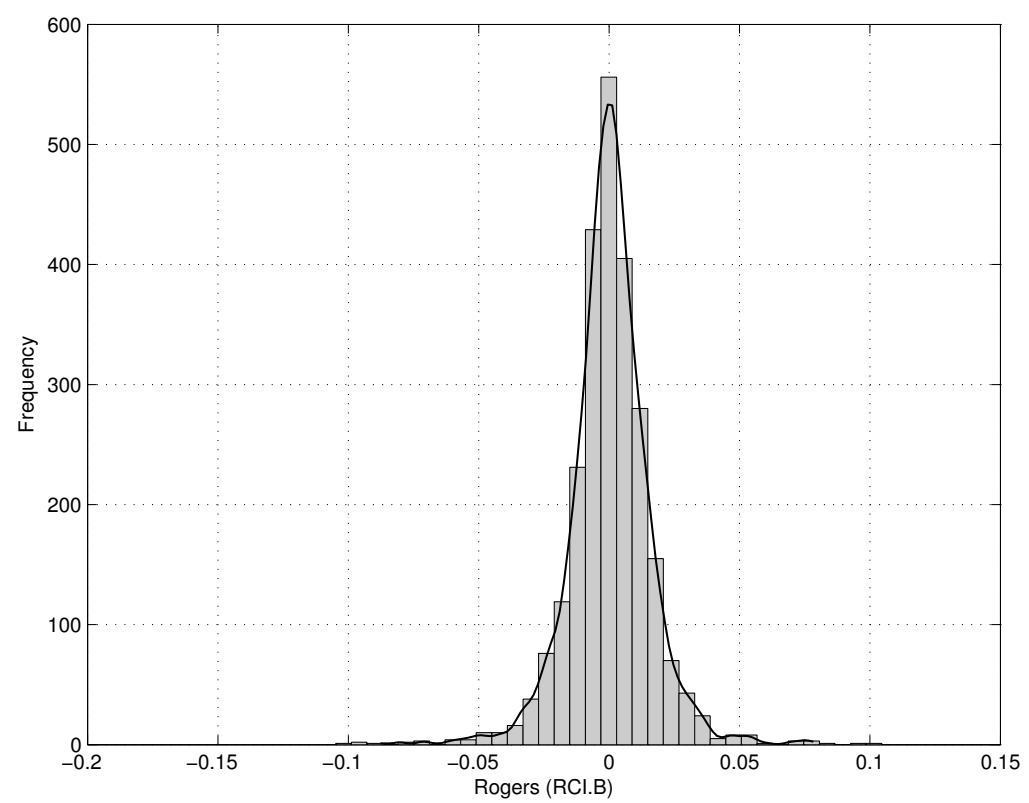

Figure 3.4: Telus (T): Histogram and Kernel Density Estimate

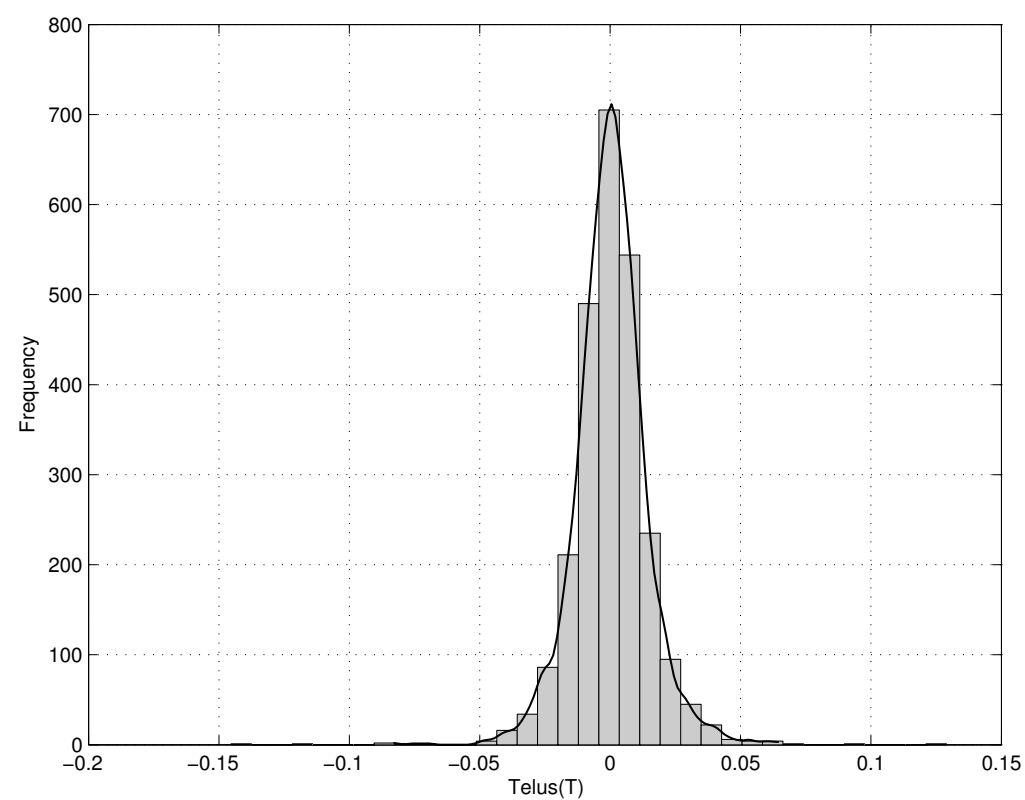


Figure 3.5: Measure of Idiosyncratic Risk

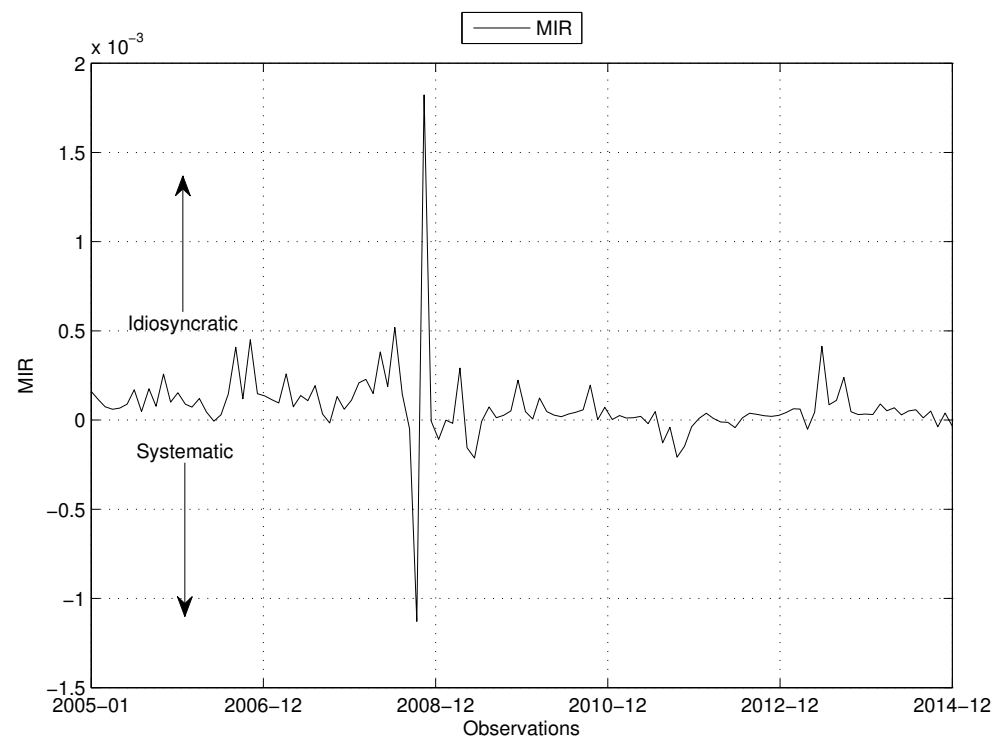


Figure 3.6: Detrended MIR Jan. 2005 -Dec. 2014.

Estimating function: OLS-long-AR

Figure (i)

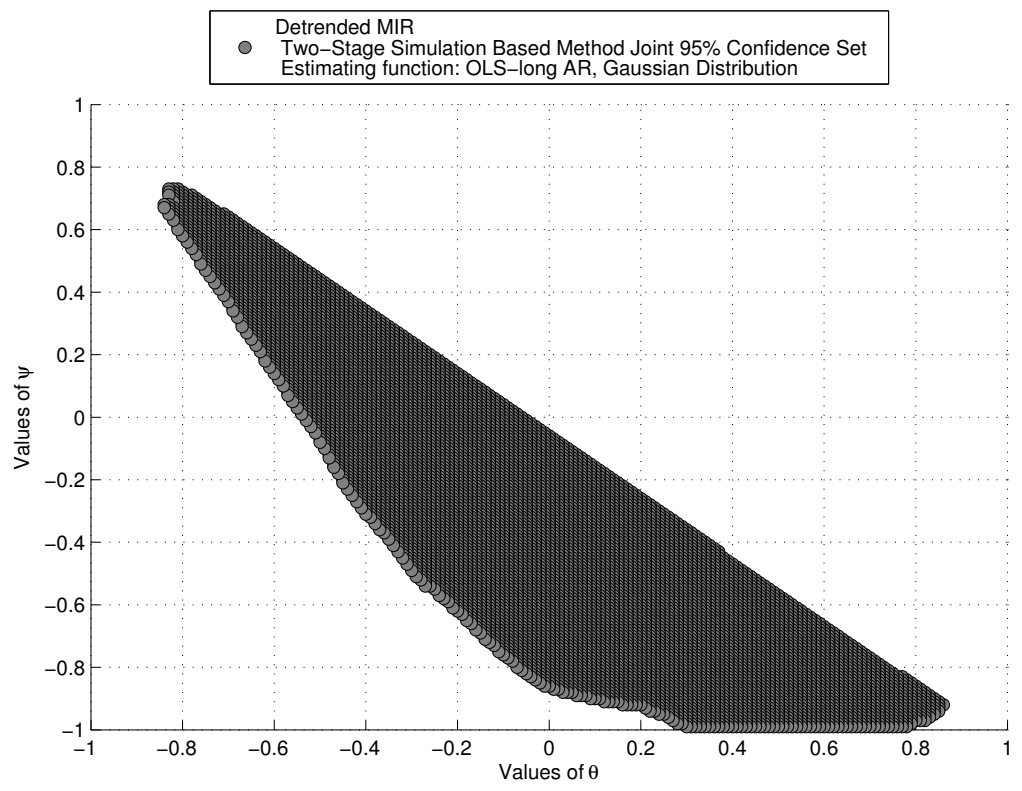

Figure (ii)

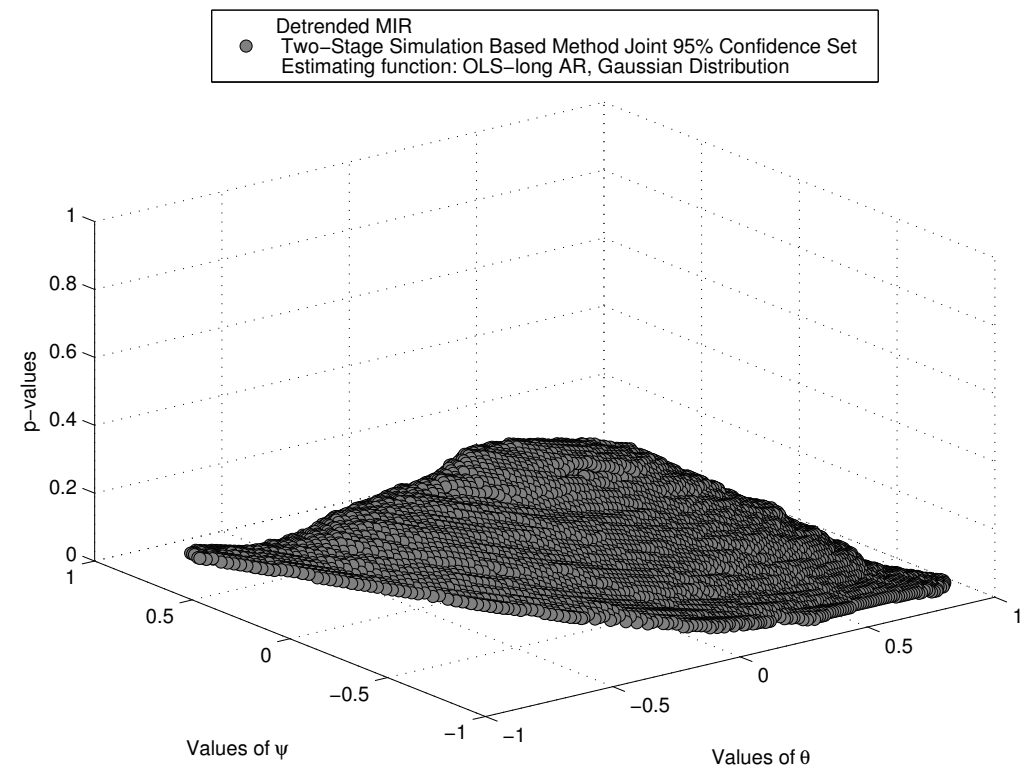


Figure (iii)

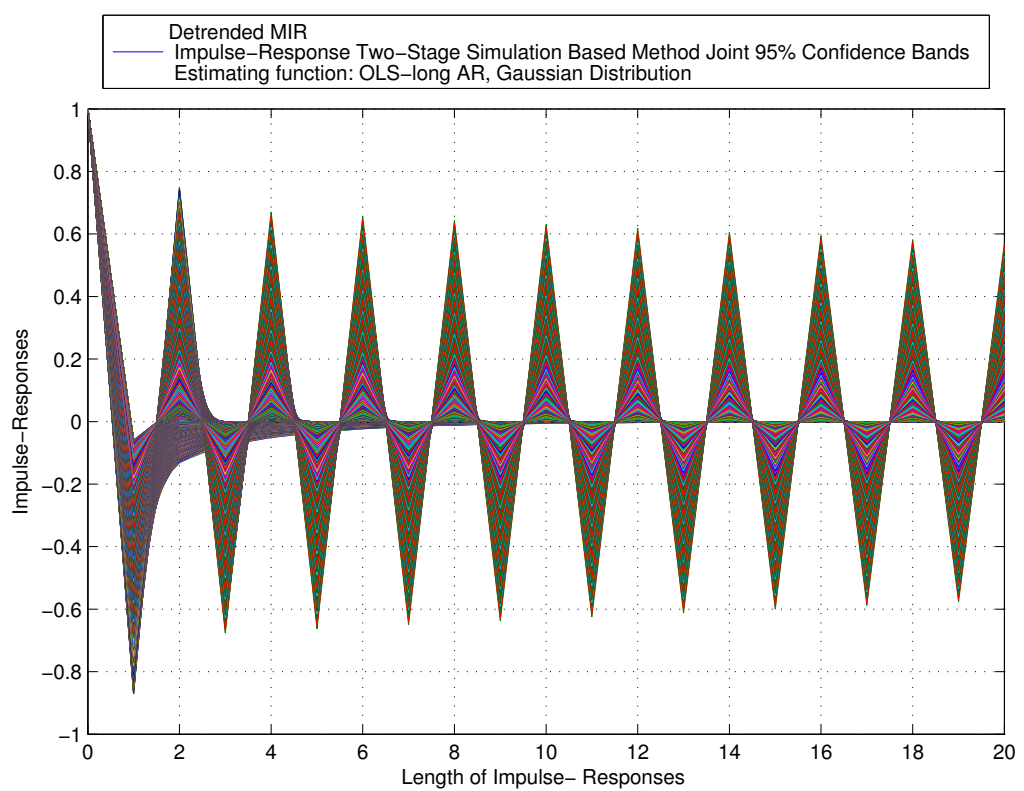

Figure (iv)

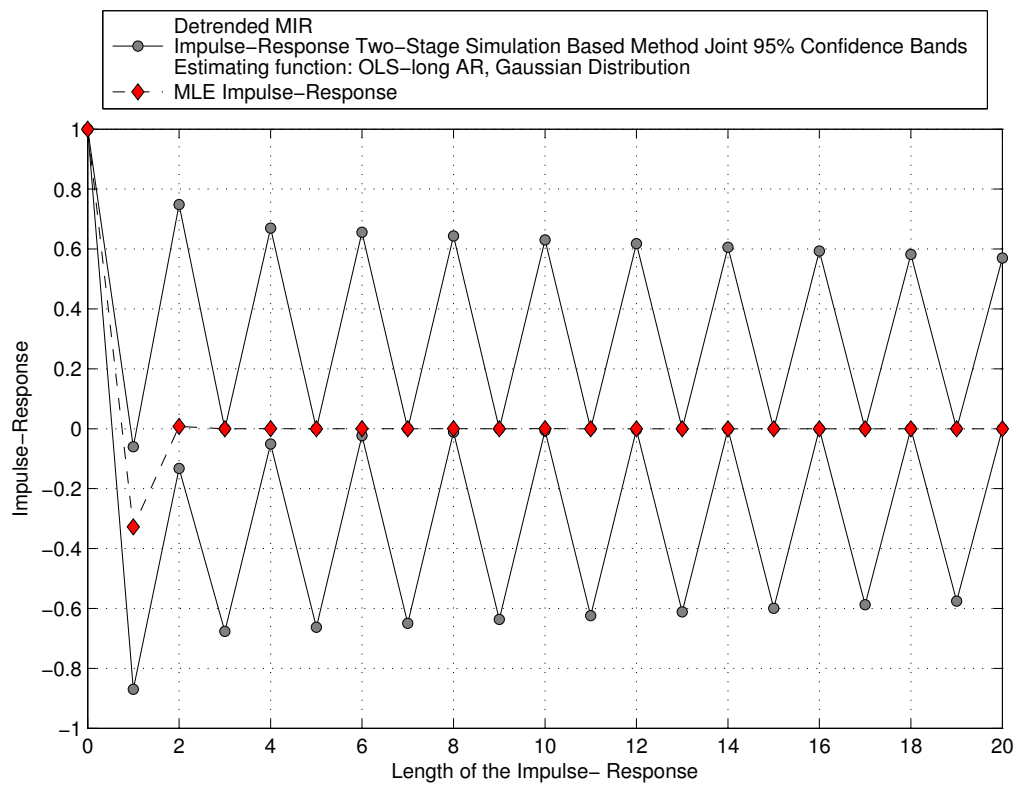


Figure 3.7: Detrended MIR Jan. 2005 -Dec. 2014.

Estimating function: Autocorrelations

Figure (i)

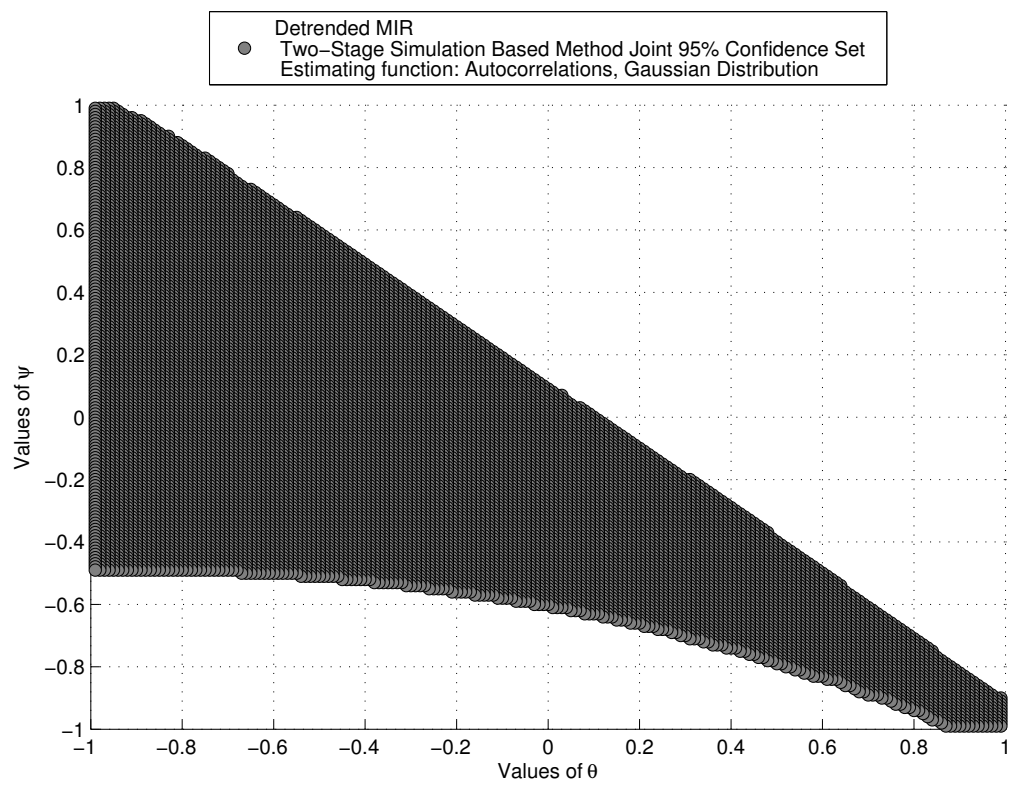

Figure (ii)

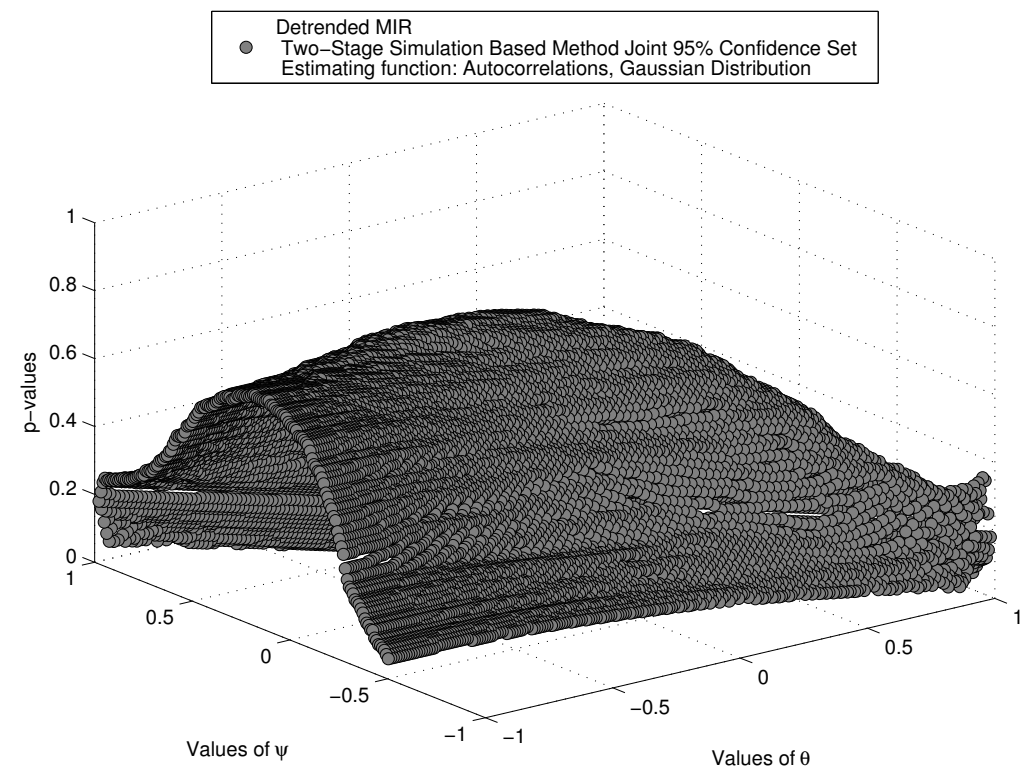


Figure (iii)

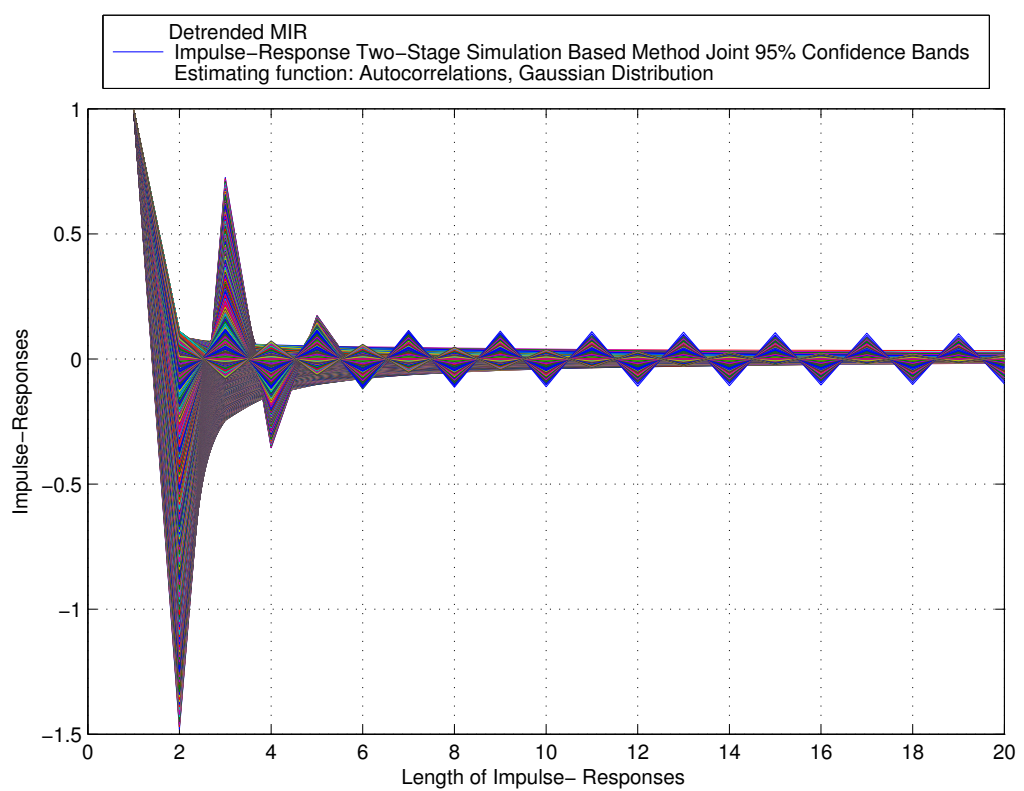

Figure (iv)

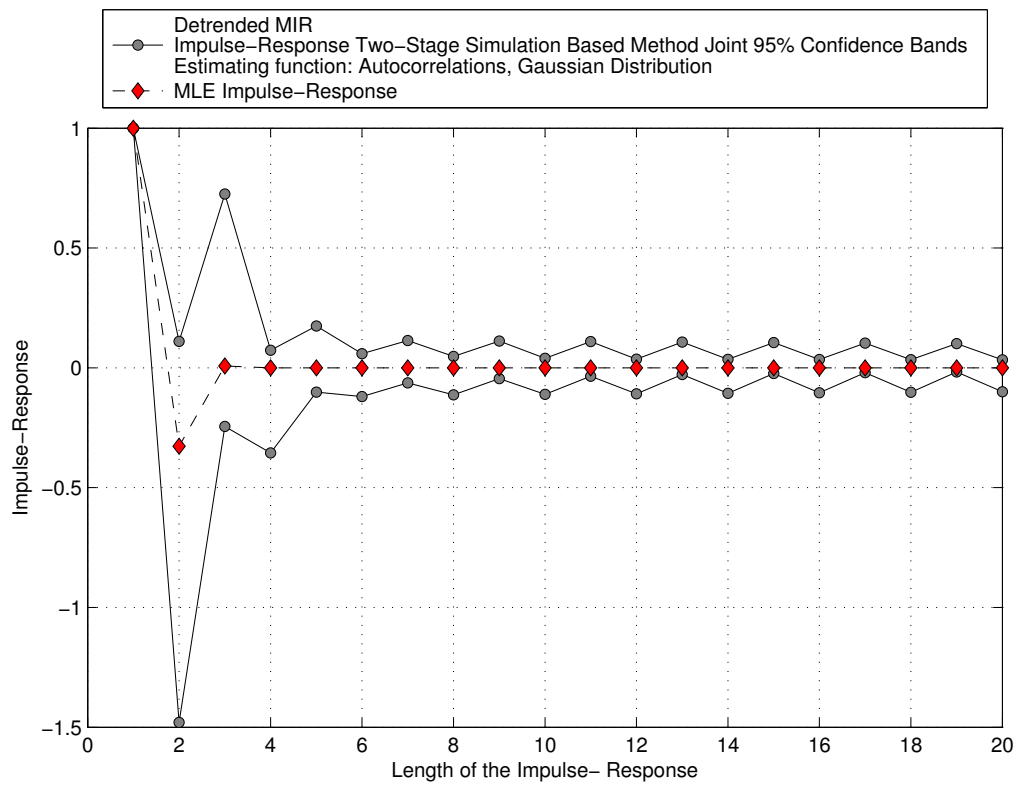


Figure 3.8: Detrended MIR Jan. 2005 -Dec. 2014. Averaging Method

Figure (i)

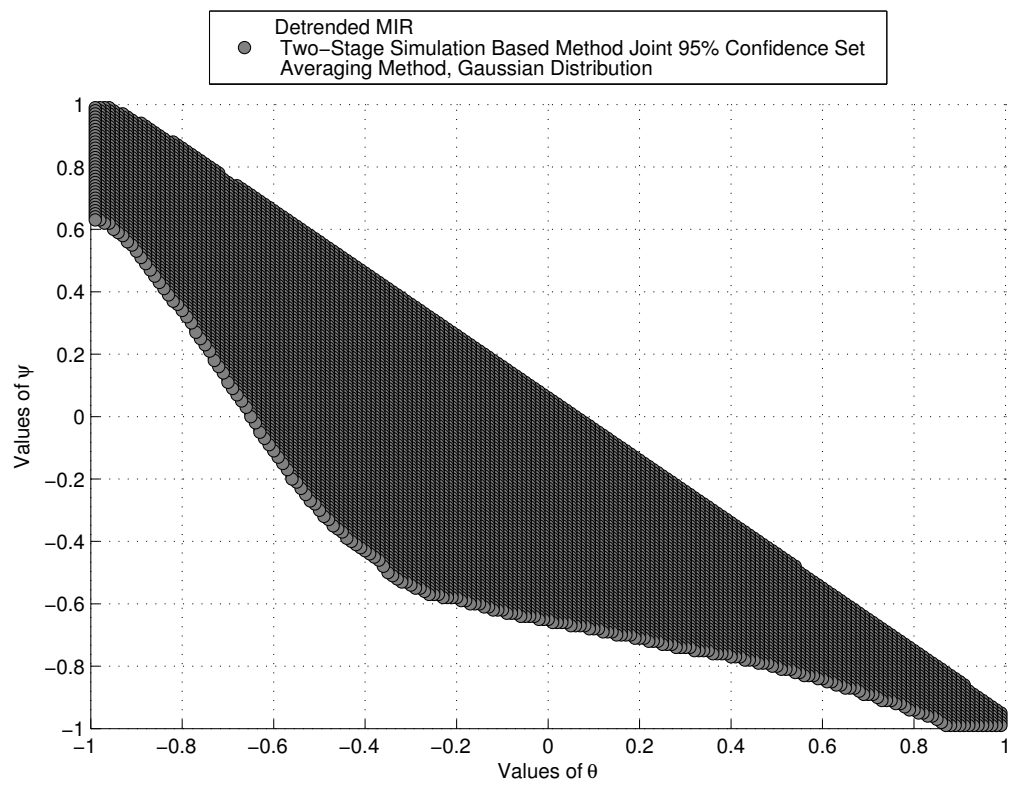

Figure (ii)

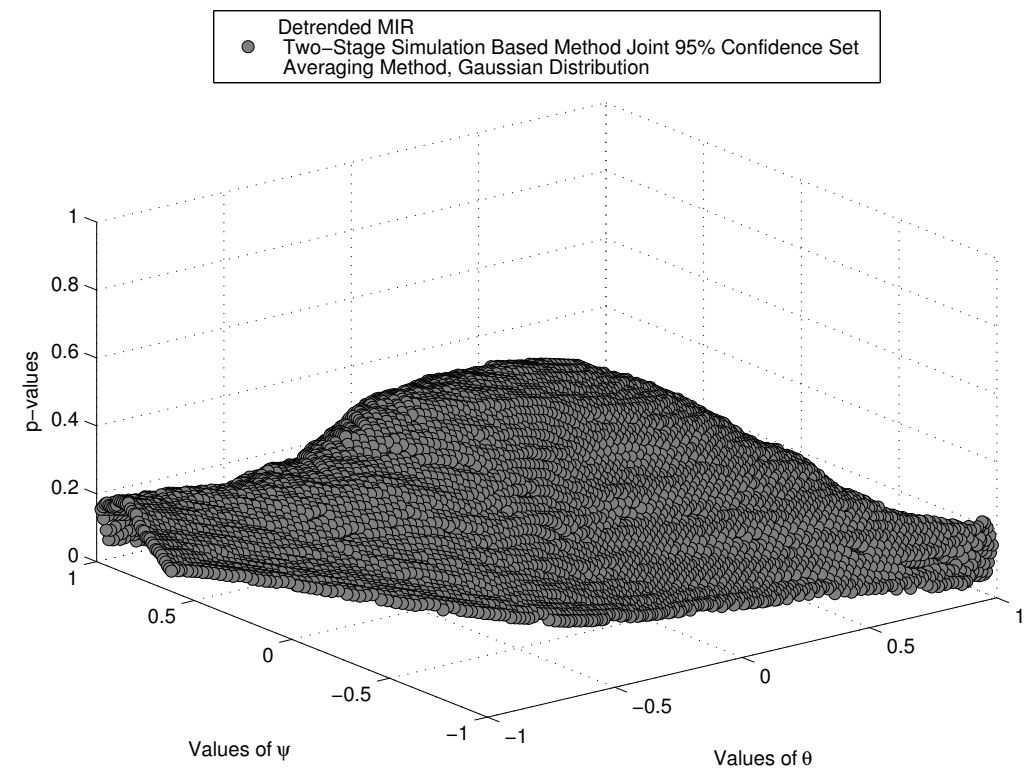


Figure (iii)

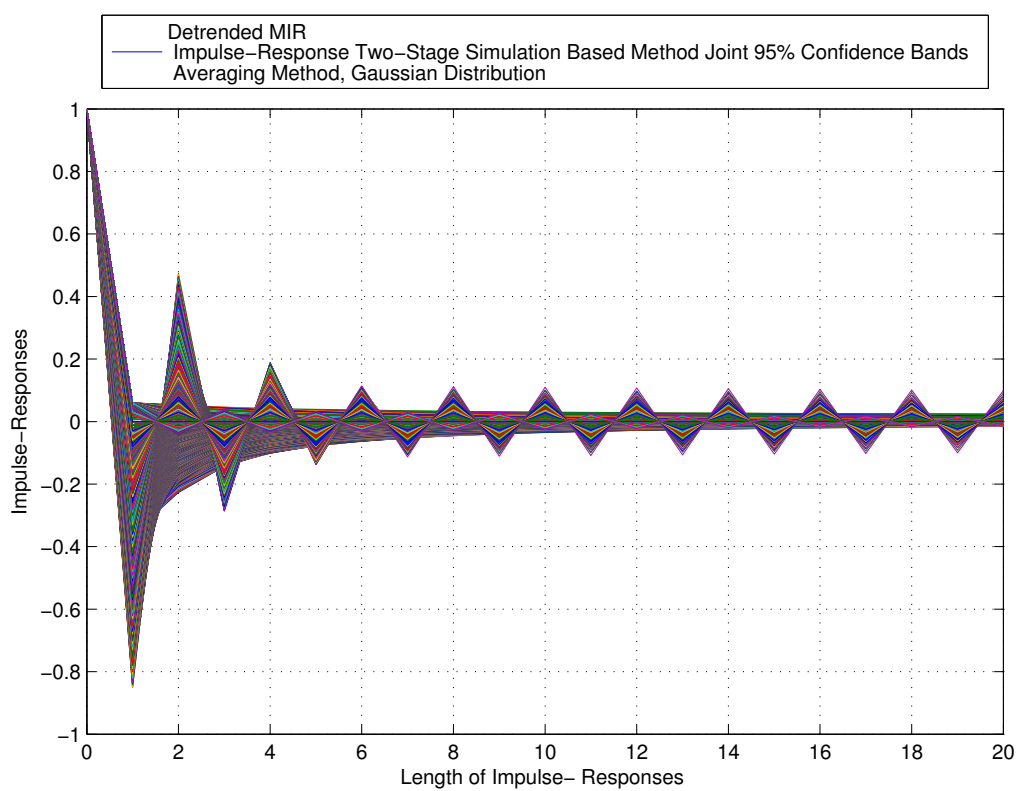

Figure (iv)

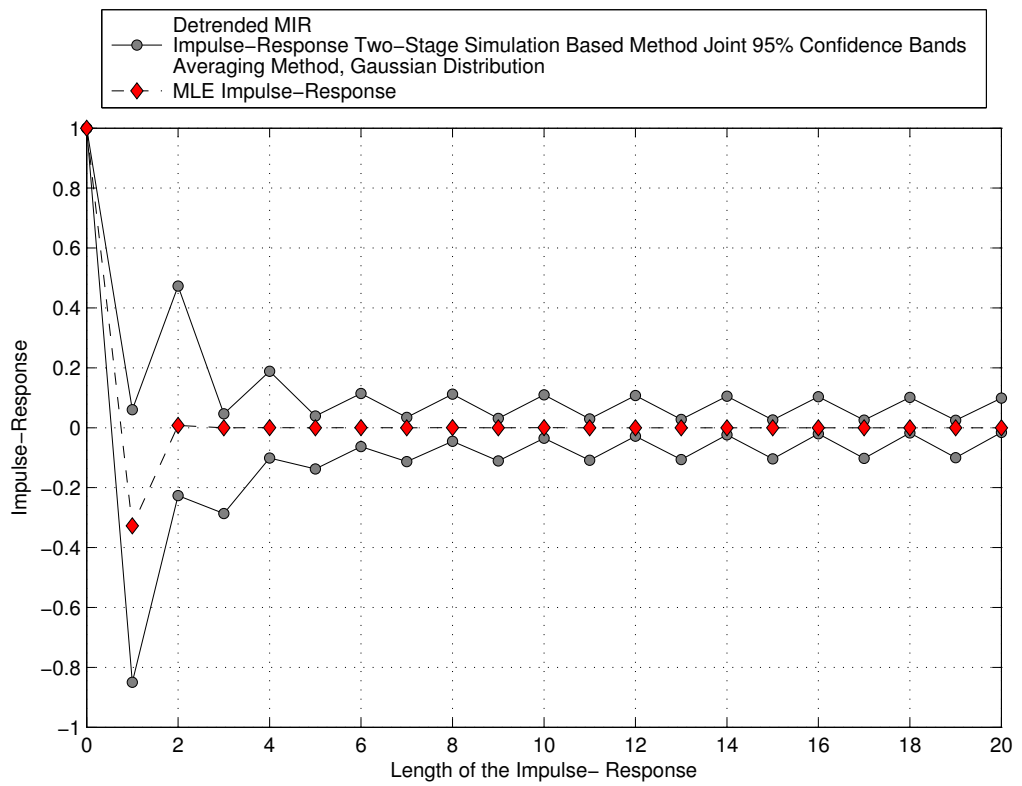

